ISAS $/ E P / 2000 / 80$

\title{
Universal Amplitude Ratios of The Renormalization Group: Two-Dimensional Tricritical Ising Model
}

\author{
D. Fioravanti ${ }^{a, b}$, G. Mussardo ${ }^{b, c}$ and P. Simon ${ }^{a, b}$ \\ ${ }^{a}$ International School for Advanced Studies, Trieste, Italy \\ ${ }^{b}$ Istituto Nazionale di Fisica Nucleare, Sezione di Trieste \\ ${ }^{c}$ Dipartimento di Fisica, Università dell'Insubria, Como, Italy
}

\begin{abstract}
The scaling form of the free-energy near a critical point allows for the definition of various thermodynamical amplitudes and the determination of their dependence on the microscopic non-universal scales. Universal quantities can be obtained by considering special combinations of the amplitudes. Together with the critical exponents they characterize the universality classes and may be useful quantities for their experimental identification. We compute the universal amplitude ratios for the Tricritical Ising Model in two dimensions by using several theoretical methods from Perturbed Conformal Field Theory and Scattering Integrable Quantum Field Theory. The theoretical approaches are further supported and integrated by results coming from a numerical determination of the energy eigenvalues and eigenvectors of the off-critical systems in an infinite cylinder.
\end{abstract}

PACS numbers: 64.60.Fr, 05.50+q,75.10Hk 


\section{Introduction}

One of the most powerful and fascinating concepts in the investigation of critical phenomena - which has successfully passed the scrutiny of both experimental and theoretical tests during the last decades - goes under the name of universality [1]. According to this principle two statistical models, which share the same symmetry of the order parameters and the dimensionality of the space of their definition, show an identical critical behavior although they may greatly differ in their microscopic realizations: near the phase transition, when the correlation length is much larger than any other microscopic scale, they appear as two representatives of the same universality class. The first characteristic of a given universality class consists of a set of critical exponents. Their values are generally given in terms of algebraic expressions of the anomalous dimensions of the relevant operators present at the critical point. In two dimensions, by the powerful methods of Conformal Field Theory (CFT) 2, 3, 4, 5] one can ascertain both the values of the critical exponents, the Operator Product Expansions (OPE) and the multi-point correlators of the relative fields.

However, a complete analysis of the class of universality should also include the description of the structure of the Renormalization Group trajectories near the critical point. The most ambitious goal would be the determination of both the scaling function which fixes the equation of state and the off-critical correlators of the various order parameters . Although the exact determination of the equation of state of a given universality class may often be a difficult task, the scaling property alone of the free-energy is nevertheless sufficient to extract numerous predictions on universal combinations of critical amplitudes. As it will become clear in section 3 , these universal combinations are pure numbers which can be extremely useful for the identification of the universality classes. In fact, the amplitude ratios are numbers which typically present significant variations between different classes of universality, whereas the critical exponents usually assume small values which only vary by a small percent by changing the universality classes. Hence the universal ratios may be ideal experimental marks of the critical scaling regime [6, 7].

In recent years, due to the theoretical progress achieved in the study of two-dimensional models, some universal ratios and other universal quantities have been computed for a large variety of bidimensional systems, such as the self-avoiding walks [8], the Ising model [9-14] or the q-state Potts model [15, 16, 17], to name few. In this paper we will concentrate our attention on the determination of the amplest possible set of universal amplitude ratios relative to the class of universality of the two-dimensional Tricritical Ising Model

\footnotetext{
${ }^{1}$ Near the critical point all quantities relative to different models of the same class of universality become identical provided an opportune rescaling of the order parameters, the external fields and the correlation length of the models is made.
} 
(TIM). Preliminary results relative to some off-critical phases of this class of universality have been presented in our previous publication [18] and the aim of this paper is twofold. First of all, to complete the list of universal amplitude ratios of the TIM presented in [18 and to perform an exhaustive analysis of all its possible phases. Secondly, to illustrate in full detail the theoretical methods which have been employed in such determination: in view of their successful applications, these techniques may be useful to analyze and to obtain similar results for other statistical models, in such a way to bridge a closer contact between theoretical and experimental results in two-dimensional physics.

Introducing the Tricritical Ising Model, from a field theoretical point of view this model may be regarded as a Landau-Ginzburg (LG) $\Phi^{6}$-theory near its tricritical point [19]. The LG terminology allows a qualitative understanding of the phase-structure of the model effortlessly. However the LG approach is often too elementary for the understanding of some remarkable symmetries present in the 2-D TIM. As a matter of fact, the bidimensional TIM is an unique example of critical phenomena: it is still sufficiently simple to be solved but at the same time it presents an extremely rich and fascinating structure of excitations which can attract the curiosity of a theorist. Depending on the direction in the phase space in which the system is moved away from criticality, one can observe, for instance, a behavior ruled by the exceptional root system $E_{7}$ [20, 21, 22] or by supersymmetry [23, 24, 25, 26] (in its exact or broken phase realization) or by an asymmetrical pair of kinks [27, 28, 29]. In addition, the description of its low-temperature phase is easily obtained from the one of its high-temperature phase because of the selfduality of the model. From the experimental point of view, a number of physical systems exhibit a tricritical Ising behavior, among them fluid mixtures or metamagnetst. Hence, there is an obvious interest in computing the ampler set of data for this class of universality and in testing the theoretical predictions versus their experimental determination. Our calculation of the universal ratios of the TIM will be performed by a combined use of results coming from Perturbed Conformal Field Theory, from the integrable structure of some of the deformations of the critical point action and also from some numerical approaches.

The paper is organized as follows. In Section 2 we briefly describe the universality class of the TIM and the symmetry properties of the theories resulting from the deformations of the critical point action made of each of the four relevant fields. Section 3 is devoted to the discussion of the scaling behavior of the singular part of the free-energy and the definition of the universal ratios obtained by considering some particular combinations of the thermodynamical amplitudes. In Section 4 we discuss the Quantum Field Theory approach to the computation of the universal ratios. The numerical method based on the

\footnotetext{
${ }^{2}$ The interest reader may find ample review of this topics in ref. 30].
} 
diagonalization of the off-critical hamiltonian obtained by truncating the Hilbert space of the conformal space is discussed in Section 5. A thorough analysis of each relevant perturbation of the TIM is performed in Section 6. Finally our conclusions are presented in Section 7. Several appendices, relative to some technical aspects of our calculations, are included at the end of the paper.

\section{The Class of Universality of the Tricritical Ising Model}

In this section we will briefly outline the main properties of the universality class of the two-dimensional Tricritical Ising model, whereby the detailed discussion of its physical properties relative to each of its perturbations is contained in Section 6 .

A possible lattice realization of the Tricritical Ising model is provided by the so-called Blume-Capel model [31]. This involves two statistical variables at each lattice site, $s_{k}-$ the spin variable - which assumes values \pm 1 and $t_{k}$ - the vacancy variable - with values 0 or 1 , which specifies therefore if the site is empty or occupied. It is characterized by the most general Hamiltonian with nearest neighbor pair interaction

$$
\mathcal{H}=-J \sum_{\langle i, j\rangle}^{N} s_{i} s_{j} t_{i} t_{j}+\Delta \sum_{i=1}^{N} t_{i}-H \sum_{i=1}^{N} s_{i} t_{i}-H_{3} \sum_{\langle i, j\rangle}^{N}\left(s_{i} t_{i} t_{j}+s_{j} t_{j} t_{i}\right)-K \sum_{\langle i, j\rangle}^{N} t_{i} t_{j} .
$$

The parameter $H$ represents an external magnetic field, $H_{3}$ an additional subleading magnetic source, $J$ the coupling between two nearest occupied sites, $\Delta$ the chemical potential coupled to the vacancies and $K$ an additional subleading energy term between them. Another possible two-dimensional lattice realization of this class of universality is provided by the so-called dilute $A_{L}$ models, discussed in [32.

By adopting a field theoretical point of view, a convenient way to analyze the universality behavior of the TIM consists in considering a Landau-Ginzburg formulation based on a scalar field $\Phi(x)$ [19]. The main advantage of this approach is an account of the $Z_{2}$ symmetry properties of each order parameter which provides an easy way of understanding the phase structure of the model, at least qualitatively. In this formulation, the class of universality of the TIM is associated to the Euclidean action

$$
\mathcal{A}=\int d^{D} x\left[\frac{1}{2}\left(\partial_{\mu} \Phi\right)^{2}+g_{1} \Phi+g_{2} \Phi^{2}+g_{3} \Phi^{3}+g_{4} \Phi^{4}+\Phi^{6}\right],
$$

with the tricritical point identified by the bare conditions $g_{1}=g_{2}=g_{3}=g_{4}=0$. In a close comparison with the Blume-Capel lattice formulation of the model, the statistical interpretation of the coupling constants is as follows: $g_{1}$ plays the role of an external magnetic field $h, g_{2}$ measures the displacement of the temperature from its critical value 
$\left(T-T_{c}\right), g_{3}$ may be regarded as a staggered magnetic field $h^{\prime}$ and finally $g_{4}$ may be thought as a chemical potential for the vacancy density.

A dimensional analysis shows that the upper critical dimension of the above LG model is $D=3$, where the tricritical exponents are expected to take their classical values, excluding logarithmic corrections. The mean field solution of the model easily shows that the LG action (2.2) has a tricritical point, i.e. a critical point where a line of a second order phase transition meets a line of a first order phase transition. Consider in fact the case where all the $Z_{2}$ odd couplings are equally set to zero. The potential in this subspace is given by

$$
V(\Phi)=g_{2} \Phi^{2}+g_{4} \Phi^{4}+\Phi^{6} .
$$

The line of a second order phase transition is identified by the condition

$$
g_{2}=0 \quad, \quad g_{4}>0
$$

whereas the line of a first order phase transition (where three degenerate vacua coexist) is obtained by the condition

$$
g_{2}>0, \quad g_{4}=-2 \sqrt{g_{2}} .
$$

Hence the point $g_{1}=g_{2}=g_{3}=g_{4}=0$ is indeed a tricritical point.

In two dimensions - the case which will mostly concern us - there are strong fluctuations of the order parameters and therefore the exponents and the amplitudes extracted by its mean field solution cannot be trusted. However, in this case one can take advantage of the powerful methods of Conformal Field Theory to obtain an exact solution of this model at criticality. In fact, the bidimensional TIM is described by the second representative of the unitary series of minimal models of CFT [2, 过, 团: its central charge is equal to $c=\frac{7}{10}$ and the Kac-table of the exact conformal weights of the scaling fields

$$
\Delta_{l, k}=\frac{(5 l-4 k)^{2}-1}{80} \quad, \quad \begin{aligned}
& 1 \leq l \leq 3 \\
& 1 \leq k \leq 4
\end{aligned}
$$

is given in Table 1 . There are six primary scalar fields $\phi_{\Delta, \bar{\Delta}}$, which close an algebra under the Operator Product Expansion

$$
\phi_{i}\left(z_{1}, \bar{z}_{1}\right) \phi_{j}\left(z_{2}, \bar{z}_{2}\right) \sim \sum_{k} c_{i j k}\left|z_{1}-z_{2}\right|^{-2\left(\Delta_{i}+\Delta_{j}-\Delta_{k}\right)} \phi_{k}\left(z_{2}, \bar{z}_{2}\right) .
$$

The skeleton form of this OPE algebra and the relative structure constants of the Fusion Rules of the TIM are in Table 2. The six primary fields can be identified with the normal ordered composite LG fields [19] (see Table 3). With respect to their properties under the $Z_{2}$ spin-reversal transformation $Q: \Phi \rightarrow-\Phi$ we have: 
1. two odd fields: the leading magnetization operator $\sigma=\phi_{\frac{3}{80},}, \frac{3}{80} \equiv \Phi$ and the subleading magnetization operator $\sigma^{\prime}=\phi_{\frac{7}{16}, \frac{7}{16}} \equiv: \Phi^{3}$ :

2. four even fields: the identity operator $1=\phi_{0,0}$, the leading energy density $\varepsilon=$ $\phi_{\frac{1}{10}, \frac{1}{10}} \equiv: \Phi^{2}:$, the subleading energy density $t=\phi_{\frac{6}{10}, \frac{6}{10}} \equiv: \Phi^{4}:$, which in metamagnets assumes the meaning of the density of the annealed vacancies, and the field $\varepsilon "=\phi_{\frac{3}{2}, \frac{3}{2}}$. The OPE of the even fields form a subalgebra of the Fusion Rules.

In the TIM there is another $Z_{2}$ transformation - the Kramers-Wannier duality $D$ - under which the fields transform as follows:

- the order magnetization operators are mapped onto their corresponding disorder operators

$$
\mu=D^{-1} \sigma D=\tilde{\phi}_{\frac{3}{80}, \frac{3}{80}} \quad, \quad \mu^{\prime}=D^{-1} \sigma^{\prime} D=\tilde{\phi}_{\frac{7}{16}, \frac{7}{16}} .
$$

- the even fields are mapped onto themselves,

$$
D^{-1} \varepsilon D=-\varepsilon, \quad D^{-1} t D=t, \quad D^{-1} \varepsilon^{\prime \prime} D=-\varepsilon ",
$$

i.e. $\varepsilon$ and $\varepsilon "$ are odd under this transformation whereas $t$ is even.

Interestingly enough, at criticality the TIM also provides a concrete realization of a supersymmetric theory since it is the first representative of the superconformal minimal models [33, 34, 35]: the even fields can be grouped into a superfield of the Neveu-Schwartz sector

$$
\mathcal{N}(z, \bar{z}, \theta, \bar{\theta})=\varepsilon(z, \bar{z})+\bar{\theta} \psi(z, \bar{z})+\theta \bar{\psi}(z, \bar{z})+\theta \bar{\theta} t(z, \bar{z}),
$$

(where $\theta$ and $\bar{\theta}$ are Grassman variables) while the magnetic fields give rise to two irreducible representations in the Ramond sector $[$. The critical superconformal LG action is given by

$$
\mathcal{A}=\int d^{2} x d^{2} \theta\left[\frac{1}{2} \mathcal{D} \mathcal{N} \overline{\mathcal{D}} \mathcal{N}+\mathcal{N}^{3}\right]
$$

with the covariant derivatives defined as

$$
\mathcal{D}=\frac{\partial}{\partial \theta}-\theta \frac{\partial}{\partial z} \quad, \quad \overline{\mathcal{D}}=\frac{\partial}{\partial \bar{\theta}}-\bar{\theta} \frac{\partial}{\partial \bar{z}} .
$$

At the critical point, the TIM can be also realized in terms of a coset construction of a Wess-Zumino-Witten model on the group $G / H$ given by $\left(E_{7}\right)_{1} \otimes\left(E_{7}\right)_{1} /\left(E_{7}\right)_{2}$ (for a general discussion on the coset model see, for instance [4]). For the central charge

\footnotetext{
${ }^{3}$ The disorder fields $\mu, \mu^{\prime}$ and the fermionic fields $\psi, \bar{\psi}$ enter the partition function of the model on a torus with twisted boundary conditions, see for instance [27] where the relative Fusion Rules are also presented.
} 
$c=c_{G}-c_{H}$ we have in fact $c=2 \times 133\left(\frac{1}{19}-\frac{1}{20}\right)=\frac{7}{10}$. Concerning the irreducible representations, for the WZW based on $\left(E_{7}\right)_{1}$ we have $\left\{\mathbf{I}, \boldsymbol{\Pi}_{6}\right\}_{1}$ with conformal dimensions $\left\{0, \frac{3}{4}\right\}$, whereas for the WZW model $\left(E_{7}\right)_{2}$ we have $\left\{\mathbf{I}, \boldsymbol{\Pi}_{1}, \boldsymbol{\Pi}_{5}, \boldsymbol{\Pi}_{6}, \boldsymbol{\Pi}_{2}\right\}_{2}$ with conformal dimensions $\left\{0, \frac{9}{10}, \frac{7}{5}, \frac{57}{80}, \frac{21}{16}\right\}$. Therefore, the conformal fields of the TIM emerge from the decomposition

$$
\begin{aligned}
& (\mathbf{I})_{1} \times(\mathbf{I})_{1}=(I)_{T I M} \otimes(\mathbf{I})_{2}+\left(\frac{1}{10}\right)_{T I M} \otimes\left(\boldsymbol{\Pi}_{1}\right)_{2}+\left(\frac{6}{10}\right)_{T I M} \otimes\left(\boldsymbol{\Pi}_{5}\right)_{2} \\
& (\mathbf{I})_{1} \times\left(\mathbf{\Pi}_{6}\right)_{1}=\left(\frac{7}{16}\right)_{T I M} \otimes\left(\boldsymbol{\Pi}_{7}\right)_{2}+\left(\frac{3}{80}\right)_{T I M} \otimes\left(\boldsymbol{\Pi}_{6}\right)_{2} \\
& \left(\mathbf{\Pi}_{6}\right)_{1} \times\left(\mathbf{\Pi}_{6}\right)_{1}=\left(\frac{3}{2}\right)_{T I M} \times(\mathbf{I})_{2}
\end{aligned}
$$

As will be discussed later, the above symmetries present at the critical point of the TIM are also useful for the investigation of some off-critical phases of the model. The four fields $\sigma, \varepsilon, \sigma^{\prime}$ and $t$ of increasing anomalous dimensions are, from a Renormalization Group point of view, all relevant operators (i.e. their conformal weight satisfies $\Delta<1$ ) and therefore they can be used to move the TIM away from criticality. To simplify the formulae below, it is convenient to adopt the compact notation $\varphi_{i}(i=1,2,3,4)$ to denote collectively all these fields, so that $\varphi_{1}=\sigma, \varphi_{2}=\varepsilon, \varphi_{3}=\sigma^{\prime}$ and $\varphi_{4}=t$. In the vicinity of the critical point the partition function of the model can be expressed by the path integral

$$
Z\left[g_{1}, g_{2}, g_{3}, g_{4}\right]=\int \mathcal{D} \phi e^{-\left[\mathcal{A}_{C F T}+\sum_{i=1}^{4} g_{i} \int \varphi_{i}(x) d^{2} x\right]} \equiv e^{-\hat{f}\left(g_{1}, g_{2}, g_{3}, g_{4}\right)}
$$

We use the notation $Z_{1}\left[g_{1}\right]=Z\left[g_{1}, 0,0,0\right], Z_{2}\left[g_{2}\right]=Z\left[0, g_{2}, 0,0\right]$, etc. for the partition functions corresponding to the individual deformations of the conformal action. An immediate result for the off-critical phases can be drawn from the symmetry properties of the fields $\varphi_{i}$. In fact, since the fields $\varphi_{1}$ and $\varphi_{3}$ are odd under the spin-reversal $Z_{2}$ symmetry, a change of the sign of the corresponding coupling constants gives rise to identical physical situations, i.e. $Z_{1}\left[g_{1}\right]=Z_{1}\left[-g_{1}\right]$ and $Z_{3}\left[g_{3}\right]=Z_{3}\left[-g_{3}\right]$. The operator $\phi_{2}$ is odd under the $Z_{2}$ duality transformation and therefore the physical situations which originate from a change of sign of this coupling constant will be related by a duality transformation. Finally the operator $\phi_{4}$ is even under both $Z_{2}$ symmetries and therefore the changing of the sign of the corresponding coupling constant will produce two distinct physical situations.

At this stage it is also useful to anticipate the nature of Quantum Field Theories which originate from each individual deformation, postponing their detailed discussion in Section 6. The QFT associated to the deformation of the field $\varphi_{1}$ alone is not integrable: numerical indications of this fact were discussed in [27]. The QFT which originates from the deformation of $\varphi_{2}$ is instead integrable and the pattern of its bound states and the scattering amplitudes are related to the hidden $E_{7}$ algebraic structure of the model [21, 22]. The deformation of the critical action by means of the field $\varphi_{3}$ produces an integrable 
field theory made of kinks, which interpolate between two asymmetric vacua [28, 29]. Finally, the $\varphi_{4}$ deformation made with a positive value of the relative coupling constant corresponds to an integrable massless RG flow between the TIM and the standard Ising model [24, 25] whereas the $\varphi_{4}$ deformation with a negative value of $g_{4}$ gives rise to an integrable massive QFT with kink excitations interpolating between three degenerate vacua [26]. Both these last QFT's give explicit realizations of a supersymmetric system in its broken and unbroken phase respectively. A useful summary of the theories resulting from each deformation can be found in Table 4 . In conclusion, excluding the magnetic $\varphi_{1}$ deformation, all the others gives rise to integrable QFT. This fact will be quite important for our future considerations.

\section{Scaling Form of the Free-Energy and Universal Ra- tios}

The scaling property of the relevant fields which span the scaling region of the TIM near its critical point allows the derivation of a large set of universal quantities which are of experimental interest. For their derivation we will consider the general case of the Tricritical Ising Model defined in a $D$ dimensional space, even though our final attention will be focalized on the two-dimensional system. At this stage of the discussion we do not take into account the eventual logarithmic corrections explicitly present in the $3-$ dimensional version of this modelf and the possible ultraviolet renormalization effects. These, however, will be considered within the context of Sections 4 and 6 .

The scaling property of the order parameters is encoded into the asymptotic form of their two-point functions

$$
\left\langle\varphi_{i}(x) \varphi_{i}(0)\right\rangle \simeq \frac{\mathcal{A}_{i}}{|x|^{4 \Delta_{i}}} \quad, \quad|x| \rightarrow 0,
$$

which therefore identifies the parameters $\Delta_{i}$ as the conformal dimensions of the fields. The standard conformal normalization of the fields is obtained by the choice $\mathcal{A}_{i}=1$. From the power law behavior of (3.1) it follows that the coupling constants $g_{i}$ behave as

$$
g_{i} \sim \Lambda^{D-2 \Delta_{i}},
$$

where $\Lambda$ is a mass scale. Therefore, moving the system away from criticality by means of one of the relevant field $\varphi_{i}$, there will be generally a finite correlation length $\xi$ which in

\footnotetext{
${ }^{4}$ On this issue the interested reader may consult for instance 30 and references therein.

${ }^{5}$ This notation is not standard for a generic $D$ dimensional system but it has the advantage of an easy comparison with formulae which are valid in 2-D systems.
} 
the thermodynamical limit scales as

$$
\xi \sim a\left(K_{i} g_{i}\right)^{-\frac{1}{D-2 \Delta_{i}}}
$$

where $a \sim \Lambda^{-1}$ may be regarded as a microscopic length scale. The terms $K_{i}$ are metric, non-universal factors which depend on the unit chosen for measuring the external sources $g_{i}$, alias on the particular realization selected for representing the universality class. In the presence of several deformations of the conformal action, the most general expression for the scaling form of the correlation length may be written as

$$
\xi=\xi_{i} \equiv a\left(K_{i} g_{i}\right)^{-\frac{1}{D-2 \Delta_{i}}} \mathcal{L}_{i}\left(\frac{K_{j} g_{j}}{\left(K_{i} g_{i}\right)^{\phi_{j i}}}\right)
$$

where

$$
\phi_{j i} \equiv \frac{D-2 \Delta_{j}}{D-2 \Delta_{i}}
$$

are the so-called crossover exponents whereas $\mathcal{L}_{i}$ are universal homogeneous scaling functions of the ratios $\frac{K_{j} g_{j}}{\left(K_{i} g_{i}\right)^{\phi_{j i}}}$. There are of course several (but equivalent) ways of writing these scaling forms, depending on which coupling constant is selected as a prefactor. In the limit where $g_{l} \rightarrow 0(l \neq i)$ but $g_{i} \neq 0$, equation (3.4) becomes

$$
\xi_{i}=a \xi_{i}^{0} g_{i}^{-\frac{1}{D-2 \Delta_{i}}} \quad, \quad \xi_{i}^{0} \sim K_{i}^{-\frac{1}{D-2 \Delta_{i}}} .
$$

Consider now the free-energy $\hat{f}\left[g_{1}, \ldots, g_{4}\right]$. This is a dimensionless quantity defined by

$$
Z\left[g_{1}, g_{2}, g_{3}, g_{4}\right]=\int \mathcal{D} \phi e^{-\left[\mathcal{A}_{C F T}+\sum_{i=1}^{4} g_{i} \int \varphi_{i}(x) d^{2} x\right]} \equiv e^{-\hat{f}\left(g_{1}, g_{2}, g_{3}, g_{4}\right)} .
$$

Assuming the validity of the hyperscaling hypothesis, in the thermodynamical limit its singular part (per unit of volume) will be proportional to the $D$ power of the correlation length. Let us denote the singular part of the free-energy for unit volume by $f\left[g_{1}, \ldots, g_{4}\right]$. Depending on which scaling form is adopted for the correlation length, we have correspondingly several (but equivalent) ways of parameterizing this quantity

$$
f\left[g_{1}, \ldots, g_{4}\right]=f_{i}\left[g_{1}, \ldots, g_{4}\right] \equiv\left(K_{i} g_{i}\right)^{\frac{D}{D-2 \Delta_{i}}} \mathcal{F}_{i}\left(\frac{K_{j} g_{j}}{\left(K_{i} g_{i}\right)^{\phi_{j i}}}\right)
$$

The functions $\mathcal{F}_{i}$ are universal homogeneous scaling functions of the ratios $\frac{K_{j} g_{j}}{\left(K_{i} g_{i}\right)^{\phi_{j i}}}$. As it will soon become clear, there is an obvious advantage in dealing with different but equivalent expressions for the free-energy: in fact, since we will be mostly concerned with the physical situations which originate from pure deformations (i.e. those obtained by keeping only one coupling constant finally different from zero), the choice of which one has to be selected naturally follows from the particular deformation which is considered. 
Let us discuss now the definition of the thermodynamical quantities related to the various derivatives of the free-energy. We will adopt the notation $\langle\ldots\rangle_{i}$ to denote expectation values computed in the off-critical theory obtained by keeping (at the end) only the coupling constant $g_{i}$ different from zero. The first quantities to consider are the vacuum expectation values (VEV) of the fields $\varphi_{j}$ which can be parameterized as

$$
\left\langle\varphi_{j}\right\rangle_{i}=-\left.\frac{\partial f_{i}}{\partial g_{j}}\right|_{g_{l}=0} \equiv B_{j i} g_{i}^{\frac{2 \Delta_{j}}{D-2 \Delta_{i}}}
$$

with

$$
B_{j i} \sim K_{j} K_{i}^{\frac{2 \Delta_{j}}{D-2 \Delta_{i}}}
$$

The above relations can be equivalently expressed as

$$
g_{i}=D_{i j}\left(\left\langle\varphi_{j}\right\rangle_{i}\right)^{\frac{D-2 \Delta_{i}}{2 \Delta_{j}}}
$$

with

$$
D_{i j} \sim \frac{1}{K_{i} K_{j}^{\frac{D-2 \Delta_{i}}{2 \Delta_{j}}}} .
$$

The generalized susceptibilities of the model are defined by

$$
\hat{\Gamma}_{j k}^{i}=\frac{\partial}{\partial g_{k}}\left\langle\varphi_{j}\right\rangle_{i}=-\left.\frac{\partial^{2} f_{i}}{\partial g_{k} \partial g_{j}}\right|_{g_{l}=0} .
$$

They are obviously symmetrical in the two lower indices. By extracting their dependence on the coupling constant $g_{i}$, they can be expressed as

$$
\hat{\Gamma}_{j k}^{i}=\Gamma_{j k}^{i} g_{i}^{\frac{2 \Delta_{j}+2 \Delta_{k}-D}{D-2 \Delta_{i}}},
$$

with

$$
\Gamma_{j k}^{i} \sim K_{j} K_{k} K_{i}^{\frac{2 \Delta_{j}+2 \Delta_{k}-D}{D-2 \Delta_{i}}} .
$$

Some of the above quantities have, of course, a very familiar meaning. For instance $\left\langle\varphi_{1}\right\rangle_{i}$ is nothing but the mean value of the magnetization in the off-critical theory defined by the $i$-th deformation while $\hat{\Gamma}_{11}^{i}$ is the associated magnetic susceptibility. Similarly, $\left\langle\varphi_{2}\right\rangle_{i}$ is the mean value of the energy along the $i$-th deformation of the critical theory and $\hat{\Gamma}_{22}^{i}$ the specific heat.

As easily seen from the above formulae, the various quantities obtained by taking the derivatives of the free-energy contain metric factors (the quantities $K_{i}$ ) which make their values not universal. However, it is always possible to consider special combinations 
thereof in such a way that all metric factors cancel out. Here we propose the consideration of the following universal ratios

$$
\begin{gathered}
\left(R_{c}\right)_{j k}^{i}=\frac{\Gamma_{i i}^{i} \Gamma_{j k}^{i}}{B_{j i} B_{k i}} ; \\
\left(R_{\chi}\right)_{j}^{i}=\Gamma_{j j}^{i} D_{j j} B_{j i}^{\frac{D-4 \Delta_{j}}{2 \Delta_{j}}} ; \\
R_{\xi}^{i}=\left(\Gamma_{i i}^{i}\right)^{1 / D} \xi_{i}^{0} ; \\
\left(R_{A}\right)_{j}^{i}=\Gamma_{j j}^{i} D_{i i}^{\frac{4 \Delta_{j}+2 \Delta_{i}-2 D}{D-2 \Delta_{i}}} B_{i j}^{\frac{2 \Delta_{j}-D}{\Delta_{i}}} ; \\
\left(Q_{2}\right)_{j k}^{i}=\frac{\Gamma_{j j}^{i}}{\Gamma_{j j}^{k}}\left(\frac{\xi_{k}^{0}}{\xi_{j}^{0}}\right)^{D-4 \Delta_{j}} ;
\end{gathered}
$$

These quantities are pure numbers which therefore characterize the universality class of the model. Their definitions closely follow and generalize the ones relative to the familiar Ising model (see, for instance [6, 12]). Other universal ratios may be defined as well and in fact some of them will be considered in Section 6 devoted to the analysis of each deformation of the critical point action. Since we will individually compute all the important quantities involved $\left(B_{i j}, \Gamma_{j k}^{i}\right.$, etc.), there is really no problem in considering other universal combinations, if one wishes to do so. It is worth emphasizing that, from an experimental point of view, it should be simpler to measure universal amplitude ratios rather than critical exponents: in fact to determine the former quantities one needs to perform several measurements at a single, fixed value of the coupling which drives the system away from criticality whereas to determine the latter, one needs to make measurements over several decades along the axes of the off-critical couplings. Moreover, although not all of them are independent, the universal ratios are a larger set of numbers than the critical exponents and therefore permit a more precise determination of the class of universality.

\section{Quantum Field Theory Approach}

Essential quantities of the universal amplitude ratios $(\overline{3.16})-(3.20)$ are the correlation length prefactor $\xi_{i}^{0}$, the VEV amplitudes $B_{j i}$ and the generalized susceptibilities $\Gamma_{j k}^{i}$. With the aim of determining these quantities, in this section we will discuss some useful results relative to the two-dimensional Quantum Field Theories (QFT) associated to the Renormalization Group flows originating by relevant deformations of the conformal action. These QFT are, after all, particular representatives of the universality class of the model and from now on we will only focalise on them for the study of the off-critical dynamics. The advantages of adopting this approach will soon become evident. 
In the following we assume the fields to be normalized according to the conformal normalization, i.e.

$$
\lim _{x \rightarrow 0}|x|^{4 \Delta_{i}}\left\langle\varphi_{i}(x) \varphi_{i}(0)\right\rangle=1,
$$

and we denote by $\mathcal{M}_{i}^{ \pm}$the QFT associated to the action

$$
\mathcal{A}=\mathcal{A}_{C F T} \pm g_{i} \int \varphi_{i}(x) d^{2} x, \quad g_{i}>0 .
$$

The coupling constant $g_{i}$ is a dimensional quantity which can be related to the lowest mass-gap $m_{i}=\xi_{i}^{-1}$ of the theory according to the formula

$$
g_{i}=\tilde{\mathcal{C}}_{i} m_{i}^{2-2 \Delta_{i}}
$$

or, equivalently

$$
m_{i}=\mathcal{C}_{i} g_{i}^{\frac{1}{2-2 \Delta_{i}}}
$$

with $\mathcal{C}_{i}=\tilde{\mathcal{C}}_{i}^{-\frac{1}{2-2 \Delta_{i}}}$. When the QFT associated to the action (4.2) is integrable, the pure number $\mathcal{C}_{i}$ can be exactly determined by means of the Thermodynamical Bethe Ansatz [36, 37]. When the theory is not integrable (this is the case for the magnetic deformation of the TIM), the constant $\mathcal{C}_{i}$ can be nevertheless determined by a numerical method, based on the so-called Truncated Conformal Space Approach [38], which will be discussed in Section 5. In conclusion, for all individual deformations of the TIM we are able to completely set the relationship which links the coupling constant to the mass-gap of the theory and therefore we are able to switch freely between these two variables.

Another set of quantities which can be fixed by QFT are the matrix elements of the order parameters, the simplest ones being the vacuum expectation values. In this case we have

$$
\left\langle\varphi_{j}\right\rangle_{i}=\tilde{B}_{j i} m_{i}^{2 \Delta_{j}}
$$

i.e.

$$
\left\langle\varphi_{j}\right\rangle_{i}=B_{j i} g_{i}^{\frac{\Delta_{j}}{1-\Delta_{i}}} \quad, \quad B_{j i}=\tilde{B}_{j i} \mathcal{C}_{i}^{2 \Delta_{j}}
$$

When the theory is integrable, the constant $\tilde{B}_{j i}$ can be fixed exactly, thanks to the results of a remarkable series of papers [39, 40]. When it is not integrable, the constant $\tilde{B}_{j i}$ can be nevertheless estimated by means of a numerical approach, first proposed in [41], which will be reviewed in Section 5. Hence, also in this case, we are able to determine completely these quantities. Let us present the exact expressions of the VEV for the three integrable deformations of the TIM, which are obtained by specializing the formulae of ref. [40]. In the expressions below, the fields are labelled by their position $(l, k)$ in the Kac table of the model (see eq. (2.6) and Table 1) and the lowest mass-gap of the different theories is simply denoted by $m$ 
- For the $\varphi_{2}$ energy deformation we have

$$
\left\langle 0_{s}\left|\Phi_{l, k}\right| 0_{s}\right\rangle=\frac{\sin \left(\frac{\pi s}{4}|5 l-4 k|\right)}{\sin \frac{\pi s}{4}}\left[\frac{5 m \pi \Gamma\left(\frac{5}{9}\right)}{2^{\frac{2}{3}} \sqrt{3} \Gamma\left(\frac{1}{3}\right) \Gamma\left(\frac{2}{9}\right)}\right]^{2 \Delta_{l, k}} \mathcal{Q}_{1,2}(5 l-4 k),
$$

where, for $|\operatorname{Re} \eta|<4, \mathcal{Q}_{1,2}(\eta)$ is given by the integral

$$
\begin{aligned}
& \mathcal{Q}_{1,2}(\eta)=\exp \left\{\int _ { 0 } ^ { \infty } \frac { d t } { t } \left(\frac{\sinh 6 t \sinh (t(\eta-1)) \sinh (t(\eta+1))}{\sinh 15 t \sinh 10 t \sinh 4 t} \times\right.\right. \\
& \left.\left.(\cosh 18 t+\cosh 8 t-\cosh 16 t+\cosh 4 t+1)-\frac{\left(\eta^{2}-1\right)}{40} e^{-4 t}\right)\right\},
\end{aligned}
$$

and is defined by its analytic continuation outside that domain. The index $s$ labels the various vacua and takes different values depending on the sign of the coupling constant $g_{2}$ : for $g_{2}<0$ the spin symmetry $\sigma \rightarrow-\sigma$ is spontaneously broken and there are two vacua identified by $s=1,3$; for $g_{2}>0$ there is a unique ground state, associated to $s=2$.

- For the $\varphi_{3}$ sub-leading magnetic deformation we have

$$
\left\langle 0_{s}\left|\Phi_{l, k}\right| 0_{s}\right\rangle=\frac{\sin \left(\frac{\pi s}{5}|5 l-4 k|\right)}{\sin \frac{\pi s}{5}}\left[\frac{4 m \pi \Gamma\left(\frac{8}{9}\right)}{2^{\frac{2}{3}} \sqrt{3} \Gamma\left(\frac{1}{3}\right) \Gamma\left(\frac{5}{9}\right)}\right]^{2 \Delta_{l, k}} \mathcal{Q}_{2,1}(5 l-4 k),
$$

where, for $|\operatorname{Re} \eta|<5, \mathcal{Q}_{2,1}(\eta)$ is given by the integral

$$
\begin{aligned}
& \mathcal{Q}_{2,1}(\eta)=\exp \left\{\int _ { 0 } ^ { \infty } \frac { d t } { t } \left(\frac{\sinh 3 t \sinh (t(\eta-1)) \sinh (t(\eta+1))}{\sinh 9 t \sinh 5 t \sinh 8 t} \times\right.\right. \\
& \left.\left.(\cosh 9 t+\cosh t-\cosh 11 t+\cosh 5 t+1)-\frac{\left(\eta^{2}-1\right)}{40} e^{-4 t}\right)\right\},
\end{aligned}
$$

and is defined by its analytic continuation outside that domain. For this deformation there are two vacua associated to the values $s=2,4$.

- For the $\varphi_{4}$ vacancy density deformation, in its massive phase we have

$$
\left\langle 0_{s}\left|\Phi_{l, k}\right| 0_{s}\right\rangle=\frac{\sin \left(\frac{\pi s}{5}|5 l-4 k|\right)}{\sin \frac{\pi s}{5}}\left[\frac{m \sqrt{\pi} \Gamma\left(\frac{7}{2}\right)}{2}\right]^{2 \Delta_{l, k}} \mathcal{Q}_{1,3}(5 l-4 k),
$$

where, for $|\operatorname{Re} \eta|<4, \mathcal{Q}_{1,3}(\eta)$ is given by the integral

$$
\mathcal{Q}_{1,3}(\eta)=\exp \left\{\int_{0}^{\infty} \frac{d t}{t}\left(\frac{\cosh 2 t \sinh (t(\eta-1)) \sinh (t(\eta+1))}{2 \cosh t \sinh 4 t \sinh 5 t}-\frac{\left(\eta^{2}-1\right)}{40} e^{-4 t}\right)\right\}
$$

and is defined by its analytic continuation outside that domain. For the massive phase of this deformation we have three vacua labelled by $s=1,2,3$. 
As discussed in Section 5, in addition to the above vacuum expectation values, a generalization of the numerical approach of ref. 41] often leads to a reasonable estimation of the matrix elements of the order parameters between the vacuum states and some of the excited states, as for instance $\left\langle 0\left|\varphi_{j}\right| A_{k}\right\rangle_{i}$ where $A_{k}$ is a one-particle state of mass $M_{k}$. These quantities will be useful for obtaining sensible approximation of the large-distance behavior of several correlators.

Another useful piece of information on the off-critical dynamics can be obtained by exploiting the properties of the stress-energy tensor $T_{\mu \nu}(x)$. In the presence of the perturbing field $\varphi_{i}$, the trace of the stress-energy tensor is different from zero and can be expressed as

$$
\Theta(x)=2 \pi g_{i}\left(2-2 \Delta_{i}\right) \varphi_{i} .
$$

The vacuum expectation value of $\Theta(x)$ is given by

$$
\langle\Theta\rangle=\tilde{w}_{i} m_{i}^{2}=w_{i} g_{i}^{\frac{1}{1-\Delta_{i}}},
$$

with $w_{i}=\tilde{w}_{i} \mathcal{C}_{i}^{2}$. As before, when the theory is integrable the constant $\tilde{w}_{i}$ can be determined exactly, otherwise it can be computed numerically tensor enters two useful sum rules which link conformal data of the ultraviolet fixed point to off-critical quantities. The first of them - called the $c$-theorem sum rule [42 - relates the central charge $c$ of the ultraviolet theory to the second moment of the two-point connected correlation function of $\Theta$

$$
c=\frac{3}{4 \pi} \int d^{2} x|x|^{2}\langle\Theta(x) \Theta(0)\rangle_{c} .
$$

For all relevant deformations, it is easy to check that the above integral is always convergent. The second sum rule - called the $\Delta$-theorem sum rule 443] - reads

$$
\Delta_{j}=-\frac{1}{4 \pi\left\langle\varphi_{j}\right\rangle_{i}} \int d^{2} x\left\langle\Theta(x) \varphi_{j}(0)\right\rangle_{i}^{c},
$$

i.e. it relates the conformal dimension $\Delta_{j}$ of the field $\varphi_{j}$ to its $\mathrm{VEV}$ and to the integral of its connected off-critical correlator with $\Theta(x)$. This time the above integral is not always convergent (the detailed analysis of its convergence may be found in the original paper [43]). Notice, however, that the $\Delta$-theorem also involves the VEV of the field $\varphi_{j}$ and it is easy to see that the divergence/convergence of the integral is always accompanied by the divergence/convergence of the VEV in such a way that the sum-rule always mantains its validity. The proof of this statement is simple: by taking the derivative of the VEV (4.6) with respect to $g_{i}$ we have in fact

$$
\frac{\partial}{\partial g_{i}}\left\langle\varphi_{j}\right\rangle_{i}=\frac{\Delta_{j}}{g_{i}\left(1-\Delta_{i}\right)}\left\langle\varphi_{j}\right\rangle_{i} .
$$

\footnotetext{
${ }^{6}$ Obviously this number can be also obtained in terms of the VEV of the field $\varphi_{i}$ and the expression of $\Theta$ given in eq. (4.13).
} 
On the other hand, the above quantity can also be computed by means of the fluctuationdissipation theorem and is given by

$$
\frac{\partial}{\partial g_{i}}\left\langle\varphi_{j}\right\rangle_{i}=-\int d^{2} x\left\langle\varphi_{i}(x) \varphi_{j}(0)\right\rangle_{c}^{i} .
$$

By using eq. (4.13) and comparing the two expressions, the divergent/convergent nature of the integral is therefore directly linked to the divergent/convergent nature of the constant $B_{j i}$ entering the VEV of the field $\varphi_{j}$. These considerations suggest that the quantity $\Delta_{j}$ on the left hand side of (4.16) is in any case obtained, also when the integral of the twopoint function $\left\langle\Theta(x) \varphi_{j}(0)\right\rangle_{c}^{i}$ diverges. In this case one needs to perform a simultaneous analytic continuation of both the integral and the corresponding VEV.

Basic quantities in the universal ratios are the generalized susceptibilities $\Gamma_{j k}^{i}$. By using equations. (3.7) and (3.14), the fluctuation-dissipation theorem provides the relation between $\hat{\Gamma}_{j k}^{i}$ and the integral of the connected correlator

$$
\hat{\Gamma}_{j k}^{i}=\int d^{2} x\left\langle\varphi_{j}(x) \varphi_{k}(0)\right\rangle_{c}^{i}
$$

The dependence on the coupling constant $g_{i}$ of these quantities can be easily extracted. In fact, the connected correlator can be parameterized as

$$
\left\langle\varphi_{j}(x) \varphi_{k}(0)\right\rangle_{c}^{i}=\frac{1}{r^{2 \Delta_{j}+2 \Delta_{k}}} Q_{j k}^{i}(m r)
$$

$(r=|x|)$. Its dependence on $m$ is obtained with a change of variable and by using the relation (4.4), we finally have $\hat{\Gamma}_{j k}^{i}=\Gamma_{j k}^{i} g_{i}^{\frac{\Delta_{j}+\Delta_{k}-1}{1-\Delta_{i}}}$ with

$$
\Gamma_{j k}^{i}=\mathcal{C}_{i}^{2 \Delta_{j}+2 \Delta_{k}-2} \int d \tau \frac{1}{\tau^{2 \Delta_{j}+2 \Delta_{k}}} Q_{j k}(\tau)
$$

Some of the above susceptibilities can be determined exactly, such as the components $\Gamma_{i k}^{i}$, whose values are provided by the $\Delta$-theorem sum rule

$$
\Gamma_{i k}^{i}=-\frac{\Delta_{k}}{1-\Delta_{k}} B_{k i}
$$

In all other cases, when an exact formula is not available, our strategy to evaluate the generalized susceptibilities will rely on two different representations of the correlators. These representations have the advantage to converge very fast in two distinct regions: the first representation is based on Conformal Perturbation Theory and allows a very efficient estimation of the correlation function in its short distance regime, while the second representation is based on the Form Factors and allows an efficient control of its large distance behavior. Due to the fast convergent nature of the two series in their respective domains, they are efficiently approximated by their lowest terms, which therefore can be evaluated with a relatively little analytical effort. These considerations obviously lead to the estimation of the integral (4.19) according to the following steps: 
1. Express the integral in polar coordinates as

$$
\hat{\Gamma}_{j k}^{i}=2 \pi \int_{0}^{+\infty} d r r\left\langle\varphi_{j}(r) \varphi_{k}(0)\right\rangle_{c}^{i},
$$

and split the radial integral into two pieces as

$$
\begin{aligned}
I=\int_{0}^{+\infty} d r r\left\langle\varphi_{j}(r) \varphi_{k}(0)\right\rangle_{c}^{i}= & \int_{0}^{R} d r r\left\langle\varphi_{j}(r) \varphi_{k}(0)\right\rangle_{c}^{i}+\int_{R}^{+\infty} d r r\left\langle\varphi_{j}(r) \varphi_{k}(0)\right\rangle_{c}^{i} \\
& \equiv I_{1}(R)+I_{2}(R) .
\end{aligned}
$$

2. Use the best available short-distance representation of the correlator to evaluate $I_{1}(R)$ as well as the best available estimate of its large-distance representation to evaluate $I_{2}(R)$.

3. Optimize the choice of the parameter $R$ in such a way to obtain the best evaluation of the whole integral. In practice, this means looking at that value of $R$ for which a plateau is obtained for the sum of $I_{1}(R)$ and $I_{2}(R)$.

In order to proceed in the above program it is useful to briefly recall the main features of the short-distance and long-distance expansions of the two-point correlation functions.

\subsection{Short-Distance Expansion}

A clear discussion on the perturbative ultraviolet renormalization of the fields and on the short-distance expansion of the two-point functions can be found in the references [43, 45]. Here we will briefly review the main results useful for our purposes.

First of all, there is a one-to-one correspondence between the fields at and away from criticality. However, renormalization effects induced by ultraviolet divergences can have the effect of expressing the off-critical fields in terms of a combination of the critical ones. Let us denote by $\tilde{\Phi}_{i}(x)$ and $\Phi_{i}(x)$ the conformal and the off-critical fields respectively. Consider the off-critical action obtained by a perturbation of a relevant (scalar) conformal field $\left(\Delta_{\tilde{\Phi}}<1\right)$

$$
\mathcal{A}=\mathcal{A}_{C F T}+g \int d^{2} x \tilde{\Phi}(x) .
$$

In a conformal perturbative evaluation of the correlators which involves one of the field $\Phi_{i}(0)$ we have

$$
\left\langle\ldots \Phi_{i}(0)\right\rangle=\left\langle\ldots \tilde{\Phi}_{i}(0)\right\rangle_{C F T}+g \int_{\epsilon<|x|<R} d^{2} x\left\langle\ldots \tilde{\Phi}_{i}(0) \tilde{\Phi}(x)\right\rangle_{C F T}+\ldots
$$

where $\epsilon$ and $R$ are the ultraviolet and the infrared cutoffs. Let us analyze first the ultraviolet behavior of the above integral. This is controlled by the OPE of the two conformal fields

$$
\tilde{\Phi}(x) \tilde{\Phi}_{i}(0)=\sum_{k} C_{\tilde{\Phi} \tilde{\varphi}}^{k}|x|^{2\left(\Delta_{k}-\Delta_{\Phi}-\Delta_{\Phi_{i}}\right)} \tilde{A}_{k}(0)
$$


and therefore the integral in (4.26) is divergent if in the above expansion there are fields $\tilde{A}_{k}(x)$ whose conformal dimensions satisfy the condition

$$
\gamma_{k} \equiv \Delta_{k}-\Delta_{\tilde{\Phi}}-\Delta_{\tilde{\Phi}_{i}}+1 \leq 0
$$

If this is the case, the off-critical renormalized field which has a finite correlator at the lowest order in $g$ is defined by

$$
\Phi_{i}=\tilde{\Phi}_{i}-g \pi \sum_{k} \frac{C_{\Phi \Phi_{i}}^{k}}{\gamma_{k}} \epsilon^{2 \gamma_{k}} \tilde{A}_{k}+\mathcal{O}\left(g^{2}\right) .
$$

Hence, due to the ultraviolet divergences there may be a mixing of the initial conformal operators with a finite numbers of fields of lower conformal dimensions.

The conformal perturbation series for the correlation functions also suffers from infrared divergences. These divergences, however, cannot be absorbed into a redefinition of the local fields and, as a result, we have a non-analytic dependence on the coupling constant $g$. This non-analytic behavior is essentially due to the non-adiabatic change of the vacuum state in passing from the Conformal Field Theory of the fixed point to the generic massive theory of the off-critical system. To overcome this difficulty, one can adopt the strategy of considering the off-critical OPE

$$
\varphi_{i}(x) \varphi_{j}(0)=\sum_{p} C_{i j}^{p}(g ; x) A_{p}(0)
$$

where the $A_{p}(x)$ belongs to a complete set of local fields of the theory (i.e. the perturbed version of the conformal fields $\left.\tilde{A}_{p}(x)\right)$ whereas the structure constants $C_{i j}^{p}(g ; x)$ are analytic in $g$ (as expected by their local nature). In this way all the non-analytic behavior of the correlation function

$$
\left\langle\varphi_{i}(x) \varphi_{j}(0)\right\rangle=\sum_{i} C_{i j}^{p}(g ; x)\left\langle A_{p}(0)\right\rangle
$$

is completely encoded inside the non-perturbative VEV's $\left\langle A_{p}(0)\right\rangle=\mathcal{A}_{p} g^{\frac{\Delta_{p}}{1-\Delta_{\Phi}}}$. Concerning the structure constants $C_{j k}^{p}(g ; x)$, by dimensional reasons they admit the expansion

$$
C_{i j}^{p}(g ; x)=r^{2\left(\Delta_{p}-\Delta_{i}-\Delta_{j}\right)} \sum_{n=0}^{\infty} C_{i, j}^{p(n)}\left(g r^{2-2 \Delta_{\Phi}}\right)^{n}
$$

where $r=|x|$ and they can be computed perturbatively $[$ in $g$. Their first order contribution is given by 45

$$
C_{i, j}^{p(1)}=-\int^{\prime} d^{2} w\left\langle\tilde{A}^{p}(\infty) \tilde{\Phi}(w) \tilde{\varphi}_{i}(1) \tilde{\varphi}_{j}(0)\right\rangle_{C F T}
$$

\footnotetext{
${ }^{7}$ The above formula has to be opportunely corrected when there is a resonance phenomenon among the fields of the conformal families.
} 
where the prime indicates a suitable infrared (large distance) regularization of the integral. It can be calculated by means of different approaches (as, for instance, a minimal subtraction scheme based on the OPE [45] or an analytic prolongation in the parameters of the integrand of (4.33), i.e. the conformal weights). As shown in [14], an efficient way to extract the finite part of the integral is provided by the Mellin transformation, discussed in Appendix A. This is the approach which we have mostly used in our calculations.

In conclusion, by using the VEV of the fields and the first approximation of the structure constants of the OPE, we can obtain a reasonable approximation for the shortdistance behavior of the connected two-point functions entering the definition of the susceptibilities in terms of the expression

$$
\begin{aligned}
\left\langle\varphi_{i}(x) \varphi_{j}(0)\right\rangle_{k}^{c}= & \sum_{p} \sum_{n=0}^{\infty} \frac{\left\langle A_{p}(0)\right\rangle_{k}}{r^{2\left(\Delta_{p}-\Delta_{i}-\Delta_{j}\right)}} C_{i j}^{p(n)}\left(g r^{2-2 \Delta_{k}}\right)^{n}+ \\
& -\left\langle\varphi_{i}\right\rangle_{k}\left\langle\varphi_{j}\right\rangle_{k} .
\end{aligned}
$$

The index $k$ indicates which perturbation is considered. Since the short-distance representation (4.31) is an expansion in the parameter $\left(\frac{r}{\xi}\right)$, a truncated form of the series (4.34) is expected to be sufficiently accurate for $r \ll \xi$. However, the convergence of the truncated series is often much better and results being sufficiently accurate also for $r \sim \xi$, as confirmed in several examples (see, for instance [11, 14, 44, 45, 46). Hence, the above truncated form (4.34) can be confidently used for the evaluation of the integral $I_{1}(R)$ in eq. (4.24). Finally, notice that the short-distance expansion of the correlators can be implemented independently on the integrable or non-integrable nature of the off-critical theory.

\subsection{Large Distance Expansion}

An efficient way to control the behavior of the correlators in the opposite regime $\left(\frac{r}{\xi}\right) \gg 1$ is provided by their spectral representation expansions. In this approach, one makes use of the knowledge of the off-critical mass spectrum of the theory to express the correlators as

$$
\left\langle\varphi_{i}(x) \varphi_{j}(0)\right\rangle=\sum_{n=0}^{\infty} g_{n}(r)
$$

where

$$
\begin{aligned}
g_{n}(r)= & \int_{\theta_{1}>\theta_{2} \ldots>\theta_{n}} \frac{d \theta_{1}}{2 \pi} \ldots \frac{d \theta_{n}}{2 \pi}\left\langle 0\left|\varphi_{i}(0)\right| A_{a_{1}}\left(\theta_{1}\right) \ldots A_{a_{n}}\left(\theta_{n}\right)\right\rangle \times \\
& \times\left\langle A_{a_{1}}\left(\theta_{1}\right) \ldots A_{a_{n}}\left(\theta_{n}\right)\left|\varphi_{j}(0)\right| 0\right\rangle e^{-r \sum_{k=1}^{n} m_{k} \cosh \theta_{k}} .
\end{aligned}
$$

\footnotetext{
${ }^{8}$ The expression (4.36) has to be suitably modified in presence of kink excitations or massless particles.
} 
$\left|A_{a_{1}}\left(\theta_{1}\right) \ldots A_{a_{n}}\left(\theta_{n}\right)\right\rangle$ are the multi-particle states relative to the excitations of mass $m_{k}$, with relativistic dispersion relations given by $E=m_{k} \cosh \theta, p=m_{k} \sinh \theta$, where $\theta$ is the rapidity variable. The spectral representation (4.36) is obviously an expansion in the parameter $e^{-\frac{r}{\xi}}$, where $\xi^{-1}=m_{1}$ is the lowest mass-gap.

Basic quantities of the large distance approach are the Form Factors (FF), i.e. the matrix elements of the operators $\varphi_{i}$ on the physical asymptotic states

$$
F_{a_{1}, \ldots, a_{n}}^{\varphi_{i}}\left(\theta_{1}, \ldots, \theta_{n}\right)=\left\langle 0\left|\varphi_{i}(0)\right| A_{a_{1}}\left(\theta_{1}\right), \ldots, A_{a_{n}}\left(\theta_{n}\right)\right\rangle .
$$

It is worth emphasizing that the above quantities are unaffected by renormalization effects since physical excitations are employed in their definitions. For scalar operators, relativistic invariance requires that the $\mathrm{FF}$ only depend on the rapidity differences $\theta_{i}-\theta_{j}$. Postponing a more detailed analysis of the analytic properties of the FF, let us first discuss the behavior of the series (4.36) for the purpose of evaluating the integral $I_{2}(R)$ in eq. (4.24).

First of all, it is convenient to order the multi-particle states entering the sum (4.36) according to the increasing values of the total sum of their masses $E_{n}^{a_{1}, \cdots, a_{n}}=\sum_{k=1}^{n} m_{k}$ so that, for $r \gg \xi$, the functions $g_{n}(r)$ behave as the decreasing sequence $g_{n}(r) \sim e^{-r E_{n}^{a_{1}, \cdots, a_{n}}}$. Apart from $g_{0}=\left\langle 0\left|\varphi_{i}(0)\right| 0\right\rangle\left\langle 0\left|\varphi_{j}(0)\right| 0\right\rangle$ relative to the VEV of the fields (which however does not enter the connected correlator), the first approximation of the correlator is given by the first term of the expansion (4.36)

$$
\begin{aligned}
\left\langle\varphi_{i}(x) \varphi_{j}(0)\right\rangle & \simeq g_{1}(r)=F_{1}^{\varphi_{i}} F_{1}^{\varphi_{j}} \int_{-\infty}^{+\infty} \frac{d \theta}{2 \pi} e^{-m_{1} r \cosh \theta}= \\
& =F_{1}^{\varphi_{i}} F_{1}^{\varphi_{j}} \frac{1}{\pi} K_{0}\left(m_{1} r\right),
\end{aligned}
$$

where $K_{0}(x)$ is the modified Bessel function. Sometimes it may occur that the oneparticle FF of the fields are zero for symmetrical reasons and, in this case, the leading approximation of the connected correlator is given by the function $g_{2}(r)$, expressed in terms of the two-particle Form Factors $F_{a_{1}, a_{2}}^{\varphi_{i}}\left(\theta_{1}-\theta_{2}\right)$. Since (4.36) is an exact expansion in $e^{-\frac{r}{\xi}}$, its truncated series to the lowest terms is expected to provide an accurate approximation of the correlators in the interval $r \gg \xi$. However, the convergence property of the truncated series is much better [8] and as a matter of fact it neatly approximates the correlator up to the region $r \sim \xi$, as has been checked in several examples (see, for instance [10, 11, 14, 45, 46, 47]). Therefore the truncated spectral series is assumed to estimate the integral $I_{2}(R)$ in eq. (4.24) with a reasonable confidence: obviously, the more terms included in the series (4.36) results in a better evaluation of the integral $I_{2}(R)$. The problem is then to determine how efficiently we can assess the matrix elements of the order parameters on the asymptotic states. Let us discuss separately the cases when the off-critical theory corresponds to a non-integrable QFT or to an integrable one. 
For a non-integrable QFT (as, for instance, the QFT resulting from the magnetic deformation of the TIM), unfortunately it is difficult to go beyond the one-particle Form Factors of the lowest particle states, $\left\langle 0\left|\varphi_{i}(0)\right| A_{k}\right\rangle$. In fact, due to creation and annihilation events in the scattering processes of these theories, i.e. to the non-elastic nature of its $S$-matrix, the FF satisfy the infinite coupled set of Watson's equations [48]

$$
\begin{aligned}
& \left.F_{\text {in }}^{\varphi}\left(\theta_{1}\right), \ldots, \theta_{n}\right)=\left\langle 0|\varphi(0)| A\left(\theta_{1}\right) \ldots A\left(\theta_{n}\right)\right\rangle_{\text {in }}= \\
& \sum_{m=0}^{\infty} \int \frac{d \theta_{1}^{\prime}}{2 \pi} \ldots \frac{d \theta_{m}^{\prime}}{2 \pi}\left\langle 0|\varphi(0)| A\left(\theta_{1}^{\prime}\right) \ldots A\left(\theta_{m}^{\prime}\right)\right\rangle_{\text {out }}\left\langle A\left(\theta_{1}^{\prime}\right) \ldots A\left(\theta_{m}^{\prime}\right) \mid A\left(\theta_{1}\right) \ldots A\left(\theta_{n}\right)\right\rangle_{\text {in }}= \\
& \sum_{m=0}^{\infty} \int \frac{d \theta_{1}^{\prime}}{2 \pi} \ldots \frac{d \theta_{m}^{\prime}}{2 \pi} F_{\text {out }}^{\varphi}\left(\theta_{1}^{\prime}, \ldots \theta_{m}^{\prime}\right) S^{n \rightarrow m}\left(\theta_{1}, \ldots, \theta_{n} \mid \theta_{1}^{\prime}, \ldots, \theta_{m}^{\prime}\right),
\end{aligned}
$$

obtained by inserting the unitary sum on the out-states in the definition of the original Form Factor. Consequently, higher-particle FF have a non-trivial analytic behavior with branch cuts at all production thresholds - which in practice precludes their exact determination. Hence, for non-integrable theories the best we can do is to estimate the large-distance expansion of the correlators only in terms of the lowest one-particle states. Moreover, the one-particle FF of these theories $\left\langle 0\left|\varphi_{i}(0)\right| A_{k}\right\rangle$ cannot be determined by first principles and for their evaluation we have to rely on some numerical determinations, as discussed in next section. Although this situation may appear disappointing from a theoretical point of view, it is worth stressing that for all practical purposes, one can reach a reasonable estimate of the integral $I_{2}(R)$ also in the non-integrable case. This can be checked, for instance, by comparing the values of the integrals - obtained by approximating the correlators by the lowest Form Factors - with their exact values obtained by the $\Delta$-theorem, when the latter applies.

For an integrable QFT, the situation is much better since, in principle, there is the possibility of determining exactly all Form Factors of the theory. For a detailed discussion of the calculation of the Form Factors in an integrable QFT, we refer the reader to the original literature [10, 45, 49, 50]. Here we simply recall the basic equations of the twoparticle FF [10] since, based on the fast convergence property expected for the spectral series [8], they will be the only terms employed in the following for approximating the correlators in their large-distance expansions (in addition, of course, to the one-particle ones).

Assume, for simplicity, that the spectrum of the integrable QFT is made of the scalar particles $A_{i}$. Let $S_{a b}(\theta)$ be the elastic scattering matrix of the particles $A_{a}$ and $A_{b}$. In this case, the two-particle Form Factor $F_{a b}^{\varphi}(\theta)$ is a meromorphic function of the rapidity difference $\theta$ satisfying the equations

$$
F_{a b}^{\varphi}(\theta)=S_{a b}(\theta) F_{a b}^{\varphi}(-\theta)
$$




$$
F_{a b}^{\varphi}(i \pi+\theta)=F_{a b}^{\varphi}(i \pi-\theta) .
$$

Let $F_{a b}^{\min }(\theta)$ be a solution of eqs. (4.40) and (4.41), free of poles and zeros in the strip $\operatorname{Im} \theta \in(0, \pi)$. By requiring asymptotic power limitation in momenta, $F_{a b}^{\varphi}(\theta)$ must be equal to $F_{a b}^{\min }(\theta)$ times a rational function of $\cosh \theta$, with the poles thereof fixed by the singularity structure of the scattering amplitude $S_{a b}(\theta)$. A simple pole in $F_{a b}^{\varphi}(\theta)$, like the one in Figure 1 induced by the simple pole of $S_{a b}(\theta)$ with a positive residue, gives rise to the equation

$$
F_{a b}^{\varphi}\left(\theta \simeq i u_{a b}^{c}\right) \simeq \frac{i \gamma_{a b}^{c}}{\theta-i u_{a b}^{c}} F_{c}^{\varphi},
$$

where $\gamma_{a b}^{c}$ is the on-shell three-particle coupling, also determined by the $S$-matrix. A more detailed analysis is, in general, required when the $S$-matrix presents higher order poles (see the discussion in [10] and Appendix D). The FF may also have kinematical poles which however do not appear at the two-particle level if the operator $\varphi(x)$ is local with respect to the fields which create the particles. In conclusion, the two-particle FF can be expressed as 10

$$
F_{a b}^{\varphi}(\theta)=\frac{\mathcal{Q}_{a b}^{\varphi}(\theta)}{D_{a b}(\theta)} F_{a b}^{\min }(\theta),
$$

where $D_{a b}(\theta)$ and $\mathcal{Q}_{a b}^{\varphi}(\theta)$ are polynomials in $\cosh \theta$ : the former is fixed by the singularity structure of $S_{a b}(\theta)$ while the latter depends on the operator $\varphi(x)$. An upper bound on the order of the polynomial $Q_{a b}^{\varphi}(\theta)$ is given in terms of the conformal dimension $\Delta_{\varphi}$. In fact

$$
\lim _{|\theta| \rightarrow \infty} F^{\varphi}(\theta) \sim e^{y_{\varphi}|\theta|}
$$

with 10

$$
y_{\varphi} \leq \Delta_{\varphi}
$$

Further equations on the polynomial $\mathcal{Q}_{a b}^{\varphi}(\theta)$ can be obtained when the field $\varphi(x)$ is proportional to the trace of the stress-energy tensor $T_{\mu \nu}(x)$. In fact, as a consequence of the conservation law $\partial_{\mu} T^{\mu \nu}=0$, the $\mathrm{FF}$ of $\Theta(x)$ for two different particles $A_{a}$ and $A_{b}$ must contain a term proportional to the Mandelstam variable $s=\left(p_{a}+p_{b}\right)^{2}$ of this state [10, 45], so that it can be factorized as

$$
\mathcal{Q}_{a b}^{\Theta}(\theta)=\left(\cosh \theta+\frac{m_{a}^{2}+m_{b}^{2}}{2 m_{a} m_{b}}\right)^{1-\delta_{a b}} P_{a b}(\theta) .
$$

Moreover, in this case we have the normalization conditions for $F_{a a}^{\Theta}$ which reads

$$
F_{a a}^{\Theta}(i \pi)=\left\langle A_{a}\left(\theta_{a}\right)|\Theta(0)| A_{a}\left(\theta_{a}\right)\right\rangle=2 \pi m_{a}^{2} .
$$

The above discussion relative to the FF (and their generalization in the case of kink excitations) will be useful in Section 6 for the determination of the generalized susceptibilities for the integrable deformations of the TIM. 


\section{Numerical Methods: Truncated Conformal Space Approach}

The Truncated Conformal Space Approach (TCSA) has been introduced by Yurov and Zamolodchikov [38 for a numerical evaluation of the non-perturbative effects relative to the off-critical models. It consists in studying the numerical spectrum of the off-critical Hamiltonian on a infinite cylinder of circumference $R$, acting on the Hilbert space of the conformal states. Once a truncation at a suitable number of states is made, the problem reduces to perform a numerical diagonalization of a finite dimensional Hamiltonian.

For the off-critical Hamiltonian we have

$$
\hat{H}=\hat{H}_{0}+\hat{V}
$$

where $\hat{H}_{0}$ is the Hamiltonian of the conformal fixed point on the cylinder and $\hat{V}=$ $g_{i} \int_{0}^{R} d v \hat{\varphi}_{i}(w)$ where $\varphi_{i}(x)$ is one of the relevant perturbation (with $w=u+i v$ is the coordinate along the cylinder and the tilde indicates quantities defined on the cylinder). By using the conformal transformation $z=e^{\frac{2 \pi}{R} w}$, the conformal theory on the cylinder is mapped onto a plane and therefore $\hat{H}_{0}$ can be expressed in terms of the usual conformal generators $L_{0}, \bar{L}_{0}$ and the central charge $c$ [51]:

$$
\begin{aligned}
\hat{H}_{0} & =\frac{2 \pi}{R}\left(L_{0}+\overline{L_{0}}-\frac{c}{12}\right) ; \\
\hat{\varphi}_{i} & =\left|\frac{2 \pi}{R}\right|^{2 \Delta_{i}} \varphi_{i} .
\end{aligned}
$$

The spectrum of $\hat{H}$ depends on the dimensionless parameter $g_{i} R^{2-2 \Delta_{i}}$ and the value of $g_{i}$ can be fixed f such that the mass gap is equal to 1 . Let $b_{m l}^{-1}$ be the inverse of the matrix $b_{m l}=\langle m \mid l\rangle$ introduced to account for the nonorthogonality of the conformal basis. Denoting by $H_{m n} \equiv b_{m l}^{-1}\langle l|H| n\rangle$ the matrix elements of the perturbed Hamiltonian, we have

$$
H_{m n}=\frac{2 \pi}{R}\left[\left(2 \Delta_{m}-c / 12\right) \delta_{m n}+2 \pi g_{i}\left(\frac{R}{2 \pi}\right)^{2\left(1-\Delta_{i}\right)} b_{m l}^{-1}\left\langle l\left|\varphi_{i}\right| n\right\rangle\right] .
$$

The matrix elements $\left\langle l\left|\varphi_{i}\right| n\right\rangle$ can be computed in terms of the structure constants of the OPE and the action of the conformal generators $L_{n}$ on the states. For the perturbation of the minimal models of CFT a numerical algorithm has been designed to compute the above matrix elements and to perform the diagonalization of the off-critical Hamiltonian by including the conformal states and their descendants up to the fifth level of the Verma module [52]. In the case of the TIM this is equivalent to truncate the number of states

\footnotetext{
${ }^{9}$ By using this normalization, we were able to determine, in particular, the constant $\mathcal{C}_{1}$ entering the relation between the coupling and the mass in the magnetic $\varphi_{1}$ deformation (see eq. (4.4)).
} 
$N$ to 228 . Once the Hamiltonian $H$ has been diagonalized for different values of $R$, one can extract the spectrum as a function of $R$, in particular the low energy eigenvalues (see, for instance Figure 2) and also their associated eigenvectors. In this way, it is possible to determine the masses of the lowest particles, several vacuum expectation values, some of the one-particle Form Factors and also some of the generalized susceptibilities. There are however certain limitations of the method, one of them due to the truncation performed in the number $N$ of the conformal states employed in the algorithm (see the discussion in [27]). In fact, all the quantities of interest are infrared data, i.e. relative to the dynamics of the system on the cylinder in the limit $R \rightarrow \infty$, which is however dominated by truncation effects. This means that in order to extract reliable infrared data one has to look at the spectrum within an interval of $R$ sufficiently large but still unaffected by truncation errors. This interval will be called the "physical window". Another limitation of the TCSA occurs when the conformal dimension $\Delta$ of the perturbing operator is such that $\Delta \geq \frac{1}{2}$. In this case, in fact, the renormalization of the operator prevents to reach a proper scaling behavior of the energy levels and the only quantities which can be extracted with reasonable confidence are the energy differences $\Delta E_{n}(R)=E_{n}(R)-E_{0}(R)$.

\subsection{Vacuum Expectation Values by TCSA}

As shown in ref. [11], the knowledge of the eigenvectors in the Truncated Conformal Space Approach allows a numerical estimation of the Vacuum Expectation Values of several order parameters. These quantities are defined by the limit

$$
B_{j i}=\lim _{R \rightarrow \infty}\left\langle\tilde{0}\left|\hat{\varphi}_{j}\right| \tilde{0}\right\rangle_{i}\left|g_{i}\right|^{-\frac{\Delta_{j}}{1-\Delta_{i}}},
$$

where $|\tilde{0}\rangle_{i}$ is the vacuum (on the cylinder) relative to the off-critical theory along the $i$-th deformation. On the other hand

$$
\left\langle\tilde{0}\left|\hat{\varphi}_{j}\right| \tilde{0}\right\rangle_{i}=\left(\frac{2 \pi}{R}\right)^{2 \Delta_{j}}\left\langle\tilde{0}\left|\varphi_{j}\right| \tilde{0}\right\rangle_{i}=\left(\frac{2 \pi}{R}\right)^{2 \Delta_{j}} \frac{\psi_{m}^{0}\left\langle m\left|\varphi_{j}\right| n\right\rangle_{i} \psi_{n}^{0}}{\psi_{m}^{0} b_{m n} \psi_{n}^{0}}
$$

where $\psi_{m}^{0}$ designs the $m^{\text {th }}$ component of the ground state vector expressed in terms of the conformal basis. Due to the truncation effects discussed above, the limit $R \rightarrow \infty$ in eq. (5.5) in practice means that one has to consider $R$ large enough such that the VEV reaches a saturation plateau. This saturation can be numerically controlled by requiring that $\left\langle 0\left|\varphi_{j}\right| 0\right\rangle_{i} \sim R^{2 \Delta_{j}}$, i.e.

$$
\frac{1}{2 \Delta_{j}} \frac{d \ln \left\langle 0\left|\varphi_{j}\right| 0\right\rangle_{i}}{d \ln R}=1
$$

By using this procedure, several VEV for different deformations of the TIM were determined in [41]. We have reproduced and confirmed the results of 41] for the two most 
relevant perturbations $\sigma$ and $\epsilon$ of the model (see Table 10). In Table 10, we have also included the exact VEV extracted from ref. 40] of the TIM perturbed by the thermal operator (for both high and low temperature phases) in order to test the feasibility of the numerical approach. As evident from this table, lower the dimension of the operator, better the accuracy of the method. This is easy to understand since the computation of the $\mathrm{VEV}$ on the cylinder is equivalent to compute the VEV at a finite-temperature, a situation analyzed in [53]. The numerical determinations of the VEV's relative to the $\varphi_{3}$ and $\varphi_{4}$ deformations turn out to be quite inaccurate for the renormalization reasons

discussed above $\left(\Delta_{3} \sim \frac{1}{2}\right.$ and $\left.\Delta_{4}>\frac{1}{2}\right)$ but their exact values can be nevertheless extracted from the results of ref. [40].

\subsection{Numerical Determination of the One-Particle Form Factors}

As discussed in Section 4, once the Form Factors of an operator are known, its correlation functions can be written as an infinite series over multi-particle states, eqs. (4.35), (4.36), and the restriction of these series to the first one-particle states already provides a reasonable approximation of their long distance behavior. These one-particle matrix elements $\left\langle 0\left|\varphi_{i}(0)\right| A_{k}\right\rangle$ can be numerically determined along the lines followed for estimating the VEV of the various operators. Namely, one has to replace the infinite volume vacuum state $\langle 0|$ with its components relative to the eigenvector of the truncated Hamiltonian, analogously for the vector relative to the one-particle state, and then use the matrix elements of the field $\varphi_{i}$ in the truncated basis. The resulting quantity finally needs to be multiplied by $\sqrt{m_{k} R}$ because the normalization of the one-particle states on a finite volume differs precisely for this factor from the one in the infinite volume. The quantity so determined, plotted versus the radius $R$ of the cylinder, presents in many cases a plateau in the region of the physical window which therefore provides its numerical estimation. As an example of such determination see Figure 3 where the matrix element $\left\langle 0\left|\varphi_{1}(0)\right| A_{1}\right\rangle_{1}$ is plotted as a function of $R$ in the case of the leading magnetic deformation of the TIM. A plateau is clearly reached for $R \approx 12$. We have performed this numerical calculation for all excited states under the two-particle threshold relative to the lowest mass gap for the first three deformations. The results are in Tables 6, 11, 12 and 17, in units of the opportune power of the coupling constants. As for the numerical determination of the VEV's, also in this case the lower the dimension of the operator, the accuracy of the method improves. Moreover, the numerical errors are usually larger for those matrix elements involving states which are closest to the threshold.

Let us conclude this section with a general remark on the one-particle Form Factors. We have already discussed that for the non-integrable deformations the knowledge of these matrix elements is crucial in obtaining at least a non-trivial estimate of the correlators 
and their determination necessarily passes through a numerical approach. However, even in the more favorable case of integrable theories, it may occur that the determination of the one-particle FF can be only obtained by a numerical approach. Despite the existence of a manageable set of recursive equations which link the various $n$-particle Form Factors in the integrable models, the solutions of these recursive equations need an initial input which cannot be often obtained even by employing the cluster property of the Form Factors [43, 54, 55]. Under this circumstance one has to necessarily resort to other methods for obtaining the one-particle FF's and the TCSA may help in this respect. An explicit example of the situation discussed above is provided for instance by the Form Factors of the operators $\varphi_{1}$ in the thermal deformation, as discussed in the Appendix D.

\subsection{Numerical Computation of the Susceptibilities}

The TCSA also allows a direct numerical estimation of the susceptibilities. Since they are defined as

$$
\hat{\Gamma}_{j k}^{i}=\frac{\partial}{\partial g_{k}}\left\langle\varphi_{j}\right\rangle_{i}=-\left.\frac{\partial^{2} f_{i}}{\partial g_{k} \partial g_{j}}\right|_{g_{l}=0}=\Gamma_{j k}^{i} g_{i}^{\frac{\Delta_{j}+\Delta_{k}-1}{1-\Delta_{i}}},
$$

one needs to numerically evaluate the derivatives of the VEV's with respect to the different couplings. Hence, a small perturbation $g_{k} \int_{0}^{R} d v \hat{\varphi}_{k}(w)$ is initially added to the Hamiltonian (5.1), with the values of the coupling constant $g_{k}$ chosen in such a way to alter the spectrum of the unperturbed theory only of small percent. To express the final result in unit of $\left|g_{i}\right|^{\frac{\Delta_{j}+\Delta_{k}-1}{1-\Delta_{i}}}$, it is convenient to write the coupling constant $g_{k}$ as

$$
g_{k}=a_{k i} g_{i}^{\frac{1-\Delta_{j}}{1-\Delta_{i}}}
$$

The next step consists in computing the expectation value of $\left\langle\hat{\varphi}_{j}\right\rangle_{i+k}$ as in eq. (5.6) by varying $a_{k i}$. As our typical sampling, we have considered 5-10 different values of $a_{k i}$ and then we have extracted the numerical estimates of various susceptibilities by a linear fit of the data in the physical window of the $R$ axis where the VEV presents a plateau. The data relative to the two strongest relevant deformations are in Tables 8, 14 and 15. Their values are reasonably close to the ones obtained by the fluctuation-dissipation theorem or to their exact values, when available from the $\Delta$-theorem sum rule. The only exceptions are those relative to the susceptibilities relative to the $\varphi_{4}$ operator, where there is a $10 \%$ mismatch. A non trivial and internal check of our estimates is given by the symmetrical relation $\Gamma_{j k}^{i}=\Gamma_{k j}^{i}$ shown by the data. As for similar calculations discussed above, this method seems however to be inappropriate for the higher dimension perturbations relative to the fields $\varphi_{3}$ and $\varphi_{4}$. 


\section{The Four Relevant Perturbations of the TIM}

In this section, we will discuss in some details the field theories relative to each individual relevant deformation of the TIM, i.e. those associated to the actions

$$
\mathcal{A}_{i}^{ \pm}=\mathcal{A}_{C F T} \pm g_{i} \int d^{2} x \varphi_{i}(x) \quad, \quad i=1, \ldots, 4
$$

The qualitative form of the effective potential relative to the different off-critical deformations of the TIM is shown in figure 4. In addition to the spectrum of the off-critical excitations, for each field theory we will present the main formulae involved in our estimation of the universal amplitude ratios of this model. Some aspects of the calculations of the correlation functions and the VEV are also discussed, refering to the appendices for all technical details. Here it is worth to comment on an interesting feature of the Conformal Perturbation Theory common to all deformations, i.e. the appearance of logarithmic terms both in some VEV and in the calculation of some susceptibilities. The origin of some of these term can be traced back to the existence of some peculiar resonance conditions involving the anomalous dimensions of this model. The first of them is given by

$$
\Delta_{1}=\Delta_{3}+\Delta_{4}-1
$$

which is equivalent to say that the scale dimensionality of the coupling $g_{1}$ equals the product of $g_{3}$ and $g_{4}$, namely $g_{1} \sim g_{3} g_{4}$. It is easy to see that this resonance condition may influence the calculation of some susceptibilities. In fact, even though each individual offcritical field theory is defined by the one-coupling action (6.1), nevertheless the calculation of the susceptibilities $\hat{\Gamma}_{j k}^{i}$ requires to consider initially the multi-coupling action

$$
\mathcal{A}=\mathcal{A}_{i}^{ \pm}+g_{j} \int d^{2} x \varphi_{j}(x)+g_{k} \int d^{2} x \varphi_{k}(x)
$$

and to take the limit $g_{j}=g_{k}=0$ only at the end of the calculation. Hence, the resonance condition $g_{1} \sim g_{3} g_{4}$ may spoil the naive form of the above action (6.3) with the presence of additional terms. Explicit examples of this phenomenon are commented in the next sections. Another resonance condition which also influence some of the calculations is given by

$$
\Delta_{6}=1+\Delta_{4}-\Delta_{2}
$$

where $\Delta_{6}=\frac{3}{2}$ is the anomalous dimension of the irrelevant field $\varepsilon "$.

\subsection{The Magnetic $\varphi_{1}$ Deformation}

This is the most relevant deformation of the TIM and the only non-integrable one. Hence, most of the results relative to this deformation are obtained by the help of the numerical 
approach. The numerical analysis of the spectrum, first performed in [27], shows that there are two different one-particle states with mass ratio $m_{2} / m_{1} \sim 1.61$ (see Figure 2). By setting the value of the first mass to be 1 , its relationship with the coupling constant $g_{1}$ is numerically determined to be

$$
m_{1}=\mathcal{C}_{1} g_{1}^{\frac{40}{77}} \approx 3.242 \ldots g_{1}^{\frac{40}{77}}
$$

The VEV of the different fields have been numerically computed and their values are in Table 5. By applying eq. (4.28), it is easy to check that there is no UV mixing of the operators for this deformation and therefore no need for their UV renormalization. In order to compute the various susceptibilities, we have decomposed $\int d^{2} x\left\langle\varphi_{i}(x) \varphi_{j}(0)\right\rangle_{1}$ into the two integrals $I_{1}(R)$ and $I_{2}(R)$, as discussed in Section 4. The UV part of the correlator has been approximated by the short range expansion (4.34) with the employed values of $\left(C_{i j}^{p}\right)_{1}^{(1)}$ reported in Table 7 . They were computed as explained in appendix B. For the IR part of the correlator, the non-integrable nature of this deformation forces us to truncate the spectral expansion to the one-particle contributions only

$$
\left\langle\varphi_{i}(x) \varphi_{j}(0)\right\rangle \approx \sum_{l=1}^{2} F_{l}^{\varphi_{i}} F_{l}^{\varphi_{j}} K_{0}\left(m_{l}|x|\right) .
$$

The numerical estimation of the one-particle Form Factors $F_{l}$, expressed in opportune units of $g_{1}$, can be found in Table 6 .

As a concrete example of the above procedure, let us consider the correlator $\left\langle\varphi_{2}(x) \varphi_{2}(0)\right\rangle_{1}$. Its UV expansion reads:

$$
\begin{aligned}
\left\langle\varphi_{2}(r) \varphi_{2}(0)\right\rangle= & r^{-4 \Delta_{2}}\left[1+c_{1} B_{41}\left(\frac{m_{1} r}{\mathcal{C}_{1}}\right)^{2}+\left(C_{22}^{1}\right)_{1}^{(1)} B_{11}\left(\frac{m_{1} r}{\mathcal{C}_{1}}\right)^{2}+\right. \\
& \left.+\left(C_{22}^{3}\right)_{1}^{(1)} B_{31}\left(\frac{m_{1} r}{\mathcal{C}_{1}}\right)^{\frac{14}{5}}+\mathcal{O}\left(\left(m_{1} r\right)^{3}\right)-\left(B_{21}\right)^{2}\left(\frac{m r}{\mathcal{C}_{1}}\right)^{4 \Delta_{2}}\right]
\end{aligned}
$$

The above expression is expected to provide an accurate approximation of the correlator up to $m_{1} r \sim 1$. Indeed, in a plot of the UV and the IR approximations of this correlator (Figure 5), a satisfactory overlap between the two curves is observed around $m_{1} r \sim 1$ : this makes us confident on the estimation of the susceptibility extracted by integrating the above correlator. The same situation occurs for the other susceptibilities and the final results are in good agreement with those extracted by the $\Delta$-theorem sum rule or their direct numerical estimation by TCSA. All these data are reported in Table 8. The only exceptions consist in the calculation of the susceptibilities $\Gamma_{34}^{1}$ and $\Gamma_{44}^{1}$ for which some care is required due to the anomalous dimensions of the fields involved and to some subtleties in the conformal perturbation expansion of $C_{i j}^{p}(g ; x)$. In these case, for instance, the naive numerical integration of the corresponding correlators cannot be performed because 
of their divergences at $r \rightarrow 0$. Let us first consider $\Gamma_{44}^{1}$. A natural way to regularise the integral $\int d^{2} x\left\langle\varphi_{4}(x) \varphi_{4}(0)\right\rangle_{1}$ which near $r \rightarrow 0$ goes as $2 \pi \int d r r^{-7 / 5}$, consists in introducing a UV cut-off $a$ so that its UV divergence is easily extracted

$$
\Gamma_{44}^{1}=\frac{5 \pi}{a^{\frac{2}{5}}}+\text { finite part } .
$$

Once this divergence is subtracted, the finite part of the integral, being cut-off independent, may be regarded as the actual regularized susceptibility. For the magnetic deformation its value is in Table 8 with $5-10 \%$ of approximation and this quantity can be used later on in the evaluation of the universal ratios. The same strategy has been also adopted for the evaluation of the susceptibilities $\Gamma_{44}^{i}$ relative to the other deformations of the TIM since the divergence of the integral is simply due to the conformal properties of the field $\varphi_{4}(x)$ and does not depend on the particular deformation considered.

The situation is different for $\Gamma_{34}^{1}$. In this case, by using the operator product expansion, it is easy to see that the integral $\int d^{2} x\left\langle\varphi_{3}(x) \varphi_{4}(0)\right\rangle_{1}$ behaves in the UV region as

$$
\int_{a}^{R} \frac{d r}{r}
$$

and therefore it presents a logarithmic divergence

$$
\begin{aligned}
\Gamma_{34}^{1} & =\frac{3 \pi}{2} B_{11} \ln \left(\frac{\Lambda}{a}\right)+G \\
& =-\frac{3 \pi}{2\left(2-2 \Delta_{1}\right)} B_{11} \ln \left(\frac{g_{1}}{g_{1}^{0}}\right)+G,
\end{aligned}
$$

where $G$ is the finite part. When $B_{11}$ is different from $0, G$ is not uniquely defined since it varies by changing the value of $g_{1}^{0}$. Hence, contrary to the previous case, the finite part of the integral cannot be used to define universal ratios although the amplitude in front of the logarithmic term is an unambiguous quantity in QFT which may enter universal combinations. It is worth pointing out that this situation is not peculiar of the TIM but it is already familiar in the context of studying the specific heat dependence in the standard two-dimensional Ising model (see, for instance [12]). Obviously the above considerations apply for all the susceptibilities $\Gamma_{34}^{i}$ relative to the other deformations of the TIM.

\subsection{The Thermal $\varphi_{2}$ Deformation}

This is an integrable deformation of the TIM [21, 22]. When $g_{2}>0$, the coupling to the thermal field moves the TIM in its high-temperature phase, where a unique $Z_{2}$ symmetric vacuum state is present. When $g_{2}<0$, we reach the low-temperature phase of the model, where there is a spontaneously breaking of the $Z_{2}$ spin symmetry and therefore two degenerate symmetric vacua. In the high-temperature phase there are 
ordinary massive particle excitations whereas in the low-temperature phase there are kink excitations and bound states thereof. The two phases are related each to the other by a duality transformation. The off-critical model possesses higher conserved charges whose spins are $s=1,5,7,9,11,13,17$ (module 18), i.e. the Coxeter exponents of the exceptional algebra $E_{7}$. The integrable structure of this deformation originates from the conformal decomposition (2.13) together with the pairing of the energy operator $\epsilon(x)$ with the adjoint representation of the WZW model on $\left(E_{7}\right)_{2}$ [20]. The existence of an infinite number of conservation laws implies the elasticity and factorization of the scattering amplitudes. These amplitudes have been computed in [21, 22] and their concise expressions can be found in Table 2 of ref. [47]. The exact mass spectrum of the excitations can be extracted from the pole structure of the $S$ matrices (see Table 9). With respect to the $Z_{2}$ spin symmetry of the model, in the high-temperature phase there are three $Z_{2}$ odd particle states (the ones relative to the masses $m_{1}, m_{3}$ and $m_{6}$ ) and four $Z_{2}$ even (those relative to the masses $m_{2}, m_{4}, m_{5}$ and $\left.m_{7}\right)$. In the low-temperature phase, the three $Z_{2}$ odd particle states become kink excitations interpolating between the two degenerate ground states whereas the four $Z_{2}$ even ones play the role of breather states. This leads, in particular, to an interesting prediction on the universal ratio of the correlation lengths above and below the critical temperature. In fact, if the correlation length is defined according to the leading exponential falling off of the spin-spin connected correlation function in the limit $|x| \gg \xi^{ \pm}$

$$
\left\langle 0\left|\varphi_{1}(x) \varphi_{1}(0)\right| 0\right\rangle_{c}^{ \pm} \sim \exp \left(-\frac{|x|}{\xi^{ \pm}}\right),
$$

(where the indices \pm refer to the high and low temperature phases respectively), from the $Z_{2}$ symmetry property of the $\sigma$ field, the self-duality of the model and the spectral representation of the above correlator we have

$$
\frac{\xi^{+}}{\xi^{-}}=\frac{m_{2}}{m_{1}}=2 \cos \frac{5 \pi}{18}=1.28557 \ldots
$$

For this deformation the relationship between the mass-gap $m_{1}$ and the coupling constant $g_{2}$ is given by $m_{1}=\mathcal{C}_{2} g_{2}^{\frac{5}{9}}$ where 37

$$
\mathcal{C}_{2}=\left(\frac{2 \Gamma\left(\frac{2}{9}\right)}{\Gamma\left(\frac{2}{3}\right) \Gamma\left(\frac{5}{9}\right)}\right)\left(\frac{4 \pi^{2} \Gamma\left(\frac{2}{5}\right) \Gamma\left(\frac{4}{5}\right)^{3}}{\Gamma\left(\frac{1}{5}\right)^{3} \Gamma\left(\frac{3}{5}\right)}\right)^{5 / 18}=3.7453728362 \ldots
$$

The VEV's of all relevant operators, both in the high or in the low temperature phases, have been computed in [40] and their values successfully compared with their numerical determination [41]: the expressions of the expectation values of the operators $\sigma$ and $\epsilon$ are finite, whereas those of the operators $\sigma^{\prime}$ and $\epsilon^{\prime}$ are naively divergent (see eq. (4.7)) and need therefore a regularization (see Appendix C). They are presented in Table 10, 
in opportune units of the associate power of the coupling constant. Concerning the UV properties of the fields, eq. (4.28) predicts that there is no UV mixing of the operators for this deformation and therefore no need to implement their UV renormalization.

Let us now turn the attention to the computation of the various susceptibilities by following the strategy explained in Section 4. The values of the coefficients $\left(C_{i j}^{p}\right)_{2}^{(1)}$ entering the UV expansion of the correlators are in Table 13. As a concrete example of these calculations, let us discuss the susceptibility amplitude $\Gamma_{11}^{2}$ relative to the correlator $\left\langle\varphi_{1}(x) \varphi_{1}(0)\right\rangle_{2}$. Its UV expansion reads:

$$
\begin{aligned}
\left\langle\varphi_{1}(r) \varphi_{1}(0)\right\rangle_{2}= & r^{-4 \Delta_{1}}\left[1+c_{5} B_{22}\left(\frac{m r}{\mathcal{C}_{2}}\right)^{\frac{1}{5}}+c_{7} B_{42}\left(\frac{m r}{\mathcal{C}_{2}}\right)^{\frac{6}{5}}+\left(C_{11}^{0}\right)_{2}^{(1)}\left(\frac{m r}{\mathcal{C}_{2}}\right)^{\frac{9}{5}}\right. \\
& \left.+\left(C_{11}^{2}\right)_{1}^{(1)} B_{22}\left(\frac{m r}{\mathcal{C}_{2}}\right)^{2}-\left(B_{12}\right)^{2}\left(\frac{m r}{\mathcal{C}_{2}}\right)^{4 \Delta_{1}}+\mathcal{O}\left((m r)^{3}\right)\right]
\end{aligned}
$$

The IR part of the correlator is approximated by taking initially into account the first four one-particle states of the spectral expansion of the correlator (see eq. (4.36))

$$
\left\langle\varphi_{i}(x) \varphi_{j}(0)\right\rangle \approx \sum_{l=1}^{4} F_{l}^{\varphi_{i}} F_{l}^{\varphi_{j}} K_{0}\left(m_{l}|x|\right) .
$$

The numerical values of the needed one-particle Form Factors are in Tables 11 and 12, relative to the high and low temperature phases respectively. Although these matrix elements are already able to reproduce with a reasonable accuracy the infrared part of the correlator, due to integrability of this deformation some two-particle Form Factors are also available and therefore they can be used to improve the estimation of the correlators. Their calculations, together with some subtleties which occur in this case, are discussed in Appendix D. The Form Factors of the operator $\epsilon(x)$, which plays the role of the trace of the stress-energy tensor for this deformation, were computed in ref. 47.

The above strategy has been applied for the estimation of all the correlators. An overlap between the UV and IR approximations of the correlators has been usually observed in the region $m_{1} r \sim 1$, which may be regarded as a consistent check of our approach. Such overlap is shown in Figure 6 for the correlator $\left\langle\varphi_{1}(x) \varphi_{1}(0)\right\rangle_{2}$.

In closing this subsection, some comments are in order for Table 13 which collects the values of the first corrections to the structure constants in the thermal deformation. Three of these coefficients contain some logarithmic dependence which however do not particularly influence the numerical approximation of the short-distance of the correlators since these are higher-order corrections. The presence of these logarithms are due to a resonance phenomenon between the conformal families, i.e. it occurs when the conformal dimensions of two operators differ for an integer number of times $1-\Delta$, where $\Delta$ is the dimension of the perturbing operator. This situation is encountered here because

$$
\Delta_{6}-\Delta_{4}=\left(1-\Delta_{2}\right) \text {. }
$$


Hence, we have for instance $\left(C_{11}^{4}\right)_{2}^{(1)}(m r)=a_{1}\left(\ln (m r)+b_{1}(m)\right)$ with the coefficient $a_{1}$ that can be computed by using the methods explained in Appendix 2. Since its algebraic expression is rather cumbersome, we prefer to report here just its numerical value $a_{1}=$ $-0.1510653 \ldots$ While $a_{1}$ is an unambigous quantity in QFT, the other term $b_{1}(m)$, on the contrary, is a scale dependent quantity. Similar resonance phenomena are also encountered in the sub-leading magnetic deformation of the TIM, which is the subject of the next subsection, where they play a more important role since they contribute to the lower orders in the UV expansion.

\subsection{The Sub-leading Magnetic $\varphi_{3}$ Deformation}

This is an integrable perturbation [28, 29]. It is generated by the less relevant magnetic field and obviously breaks the $Z_{2}$ spin symmetry of the critical point since the field $\varphi_{3}$ is a $Z_{2}$ odd operator. The resulting massive theory presents some interesting features, as first outlined in ref. [27]. The theory presents two degenerate albeit asymmetrical vacua (denoted by $\left|0_{2}\right\rangle$ and $\left|0_{4}\right\rangle$ ). There are two massive kink excitations of mass $m$ and one breather bound state thereof with the same mass. The exact $S$-matrix of this theory has been computed and analyzed in [29]. The relationship between the coupling constant $g_{3}$ and the mass gap of the theory is provided by $m=\mathcal{C}_{3} g_{3}^{\frac{8}{9}}$ with the constant $\mathcal{C}_{3}$ given by [37]

$$
\mathcal{C}_{3}=\frac{\sqrt{3} \Gamma\left(\frac{1}{3}\right) \Gamma\left(\frac{5}{9}\right)}{\pi \Gamma\left(\frac{8}{9}\right)}\left[\frac{\pi^{2} \Gamma^{2}\left(\frac{11}{16}\right) \Gamma\left(\frac{1}{4}\right)}{\Gamma^{2}\left(\frac{5}{16}\right) \Gamma\left(\frac{3}{4}\right)}\right]^{\frac{4}{9}}=4.92779064 \ldots
$$

The VEV's of relevant operators $\varphi_{1}, \varphi_{2}, \varphi_{3}$ have been exactly computed in 40]. The expression of the VEV's $\left\langle 0_{s}\left|\varphi_{4}\right| 0_{s}\right\rangle, s=2,4$ given by eq. (4.9) are a priori divergent and need to be regularized. As shown in Appendix $\mathrm{C}$ and also discussed below, they present a logarithmic dependence on the coupling constant $g_{3}$. The complete set of VEV, expressed in unit of the appropriate power of the coupling constant, are in Table 16 . Notice that, according to the formula (4.28), the only field which requires an ultraviolet renormalization is precisely the field $\varphi_{4}(x)$ which mixes (logarithmically) with the field $\varphi_{1}(x)$ under perturbation theory.

The application of the $\Delta$-theorem sum rule allows the exact determination of the four susceptibilities $\Gamma_{3 j}^{3}$ (as already discussed in Section 6.1, the susceptibility $\Gamma_{34}^{3}$ contains a logarithmic dependence on the coupling constant $g_{3}$ ). Also for this deformation we have followed the general strategy explained in Section 4, i.e. we have first pursued the matching between the UV and the IR expansions of the correlators and then we have performed their integration for extracting the susceptibilities. Let us discuss the correlator $\left\langle\varphi_{1}(r) \varphi_{1}(0)\right\rangle_{3}$ to exemplify some new difficulties arising in the perturbative evaluation. Its 
UV expansion is given by

$$
\begin{aligned}
\left\langle\varphi_{1}(r) \varphi_{1}(0)\right\rangle_{3} & =r^{-4 \Delta_{1}}\left(1+c_{5} B_{23}\left(\frac{m r}{\mathcal{C}_{3}}\right)^{\frac{1}{5}}+c_{7} B_{43}\left(\frac{m r}{\mathcal{C}_{3}}\right)^{\frac{6}{5}}+\left(C_{11}^{1}\right)_{3}^{(1)} B_{13}\left(\frac{m r}{\mathcal{C}_{3}}\right)^{\frac{6}{5}}\right. \\
& \left.+\left(C_{11}^{3}\right)_{3}^{(1)}(r) B_{33}\left(\frac{m r}{\mathcal{C}_{3}}\right)^{2}+\ldots-\left(B_{13}\right)^{2}\left(\frac{m r}{\mathcal{C}_{3}}\right)^{4 \Delta_{1}}\right) .
\end{aligned}
$$

There are two different sources of problems in this expansion. First of all, the VEV $\left\langle\varphi_{4}\right\rangle_{3}$ contains a logarithm dependence on the coupling and a UV regularization is needed. By using eq. (6.10) and the $\Delta$-theorem sum rule

$$
B_{43}=-\frac{\left(1-\Delta_{3}\right)}{\Delta_{4}} \Gamma_{34}^{3}
$$

it can be easily shown that

$$
B_{43}=-\frac{3 \pi\left(1-\Delta_{3}\right)}{2 \Delta_{4}} B_{13}\left(\ln \frac{\Lambda}{\epsilon}+G_{3}\left(\frac{\Lambda}{\epsilon}\right)\right)=\frac{3 \pi}{4 \Delta_{4}} B_{13}\left(\ln \frac{g_{3}}{g_{3}^{0}}+\tilde{G}_{3}\left(\frac{g_{3}}{g_{3}^{0}}\right)\right)
$$

where $\epsilon$ is some arbitrary UV cut-off and $\Lambda \sim \xi_{3}^{0}$. Secondly, the coefficient $\left(C_{11}^{1}\right)_{3}^{(1)}$ contains some logarithmic divergences due to resonance problems already encountered in the thermal perturbation and cannot be calculated by using the Mellin transformation method in the usual manner. However, the Mellin transformation method can be properly generalized to obtain the correct logarithmic term, as we show in the following. $\left(C_{11}^{1}\right)_{3}^{(1)}(r)$ is defined as usual by the regularized integral (notice that we have restored the dependence on $r$ )

$$
\left(C_{11}^{1}\right)_{3}^{(1)}(r)=-\int^{\prime} d^{2} z\left\langle\varphi_{1}(\infty) \varphi_{1}(r) \varphi_{3}(z) \varphi_{1}(0)\right\rangle
$$

Using notation of Appendix A, $\left(C_{11}^{1}\right)_{3}^{(1)}(r)$ may be regarded as the regularized limit $s \rightarrow 0$ of

$$
\begin{aligned}
\tilde{G}(2-s ; r) & =\int d^{2} z|z|^{-s}\left\langle\varphi_{1}(\infty) \varphi_{1}(r) \varphi_{3}(z) \varphi_{1}(0)\right\rangle \\
& =r^{-s} \iint d^{2} z d^{2} u|u|^{2 a}|u-1|^{2 b}|u-z|^{2 c}|z|^{2 d-s}|z-1|^{2 e}
\end{aligned}
$$

where the coefficients $a, b, c, d, e$ are expressed in terms of the weights by the Coulomb gas formalism and $s$ is the Mellin transformation parameter. After putting $r=1$ in the previous equation, we have

$$
\tilde{G}(2-s ; 1)=-\frac{\frac{3 \pi}{8} c_{1}}{s}+\mathcal{O}(1)
$$

i.e. there is a first order pole which does not allow us to calculate $\left(C_{11}^{1}\right)_{3}^{(1)}$ in the usual manner. However, by multiplying the previous expression with the expansion (see (C.48)

$$
r^{-s}=m^{s}(m r)^{-s}=\left[1-s \ln m r+\mathcal{O}\left(s^{2}\right)\right],
$$


where $\mathrm{m}$ is an arbitrary mass scale, we obtain the expansion in $s$

$$
\tilde{G}(2-s ; r)=-\frac{\frac{3 \pi}{8} c_{1}}{s}+\frac{3 \pi}{8} c_{1} \ln m r+\mathcal{O}(1)
$$

Hence, $\left(C_{11}^{1}\right)_{3}^{(1)}(r)$ may be taken as the regularized version of the previous expression, i.e. the one obtained by discarding the simple pole divergence

$$
\left(C_{11}^{1}\right)_{3}^{(1)}(r)=\frac{3 \pi}{8} c_{1}(\ln (m r)+\text { const }),
$$

where the constant term clearly depends on the scale $m$. By looking at eq. (6.21) and by taking into account the dependence of $a, b, c, d, e$ on the weights of the primary fields, it is easy to see that a shift of the parameter $\xi$, defined in eq. (C.31), $\xi=\frac{p}{p^{\prime}-p} \rightarrow \xi+\epsilon$ would

have led to the same result, with $\epsilon$ playing the rôle of $s$. The two terms in $\left(\frac{m r}{\mathcal{C}_{3}}\right)^{\frac{6}{5}}$ lead in the correlator to

$$
\frac{3 \pi}{8} c_{1} B_{13}\left(\frac{m r}{\mathcal{C}_{3}}\right)^{\frac{6}{5}}(\ln (m r)+A(m))
$$

where $A$ is a cut-off dependent quantity which can be determined by requiring the matching between the infrared expansion of the correlator with its UV part. The infrared part is approximated by the one-particle Form Factors of the magnetic and thermal operators (which are reported in Table 17). There is a non-trivial consistency check of the above procedure. Indeed, the correlation function can be computed in the two different vacua $\left|0_{2}\right\rangle$ and $\left|0_{4}\right\rangle$. Once the quantity $A$ has been fixed by computing the correlator $\left\langle 0_{2}\left|\varphi_{1}(r) \varphi_{1}(0)\right| 0_{2}\right\rangle$, the same $A$ should also work for the other correlator $\left\langle 0_{4}\left|\varphi_{1}(r) \varphi_{1}(0)\right| 0_{4}\right\rangle$, as it is indeed the case.

The above procedure has been also employed for the correlators $\left\langle\varphi_{2}(r) \varphi_{2}(0)\right\rangle_{3}$ and for $\left\langle\varphi_{1}(r) \varphi_{2}(0)\right\rangle_{3}$. For this deformation, however, we were unable to reach any definite result on those correlators involving the $\varphi_{4}$ operator because its one-particle FF were completely inaccurate, thus preventing a reliable evaluation of the infrared part of the correlators. The results for all the susceptibilities we were able to compute for this deformation are in Table 19 .

\subsection{The Vacancy Density $\varphi_{4}$ Deformation}

The field theories originated by the perturbation $t(x)=\varphi_{4}(x)$ with $g_{4}>0$ and $g_{4}<0$ are both integrable [26]. The most elegant way to get an insight on these quantum field theories is to use the supersymmetric formulation of the TIM [19, 23, 26]. Since the field $t(x)$ is the top component of the superfield (2.10), the off-critical dynamics may be described by the action

$$
\mathcal{A}=\int d^{2} x d^{2} \theta\left[\frac{1}{2} \mathcal{D} \mathcal{N} \overline{\mathcal{D}} \mathcal{N}+\mathcal{N}^{3}+g_{4} \mathcal{N}\right]
$$


After eliminating $t(x)$ by its algebraic equation of motion, the interaction terms of the above lagrangian are given by

$$
\bar{\psi} \psi \epsilon+\frac{1}{2}\left(\frac{1}{2} \epsilon^{2}+g_{4}\right)^{2}
$$

Hence, for $g_{4}>0$ the ground state energy is nonzero and supersymmetry is spontaneously broken: the scalar field acquires a mass whereas the fermionic field remains massless and plays the role of goldstino. This is nothing but the Majorana fermionic field of the familiar two-dimensional Ising model which is in fact the ending point of the massless Renormalization Group flow originating from the $g_{4}>0$ deformation [23]. On the massless Majorana fermion of the Ising model supersymmetry is implemented non-linearly. The exact massless $S$-matrix has been computed in [24] and the related massless Form Factors in [25]. The crossover phenomena, where the exponents characterizing the leading singularity of an observable change from its tricritical to its critical value, have been studied in [25]. Along this flow, the conformal dimension of the magnetization field changes from its tricritical value $\frac{3}{80}$ to $\frac{1}{16}$ of the Ising fixed point, the conformal dimension of the energy operator varies from $\frac{1}{10}$ to $\frac{1}{2}$ and finally the conformal dimension of the vacancy density operator changes from $\frac{6}{10}$ to 2 , since this operator becomes - at the end of the Renormalization Group flow - a descendent of the Identity operator. The sub-leading magnetization operator also becomes at the end of the RG flow a descendent of the magnetization field of the Ising model. For the massless nature of this theory we will not compute the relative universal amplitude ratios.

For $g_{4}<0$ the ground state energy vanishes therefore supersymmetry is unbroken and both the scalar and the fermion fields become massive. As argued in [26], to describe the dynamics of the system it is more convenient to adopt the usual Landau-Ginzburg potential in terms of the order parameter $\sigma(x)$. This potential presents three-fold degenerate vacua, labelled by $\left|0_{-1}\right\rangle,\left|0_{0}\right\rangle,\left|0_{+1}\right\rangle$, where $\left|0_{0}\right\rangle$ corresponds to the disordered vacuum and $\left|0_{ \pm 1}\right\rangle$ to the two disordered vacua, symmetrically placed with respect to the origin (see Figure 4). The elementary excitations in this phase are massive kinks, which interpolate between the ordered vacuum and the disordered vacua and are denoted by $K_{0 \pm 1}$ or $K_{ \pm 10}$. The associated scattering theory has been discussed in [26, 56].

Let us consider the amplitude ratios for this deformation. The VEV of $\left\langle\varphi_{1}\right\rangle_{4}$ and $\left\langle\varphi_{2}\right\rangle_{4}$ can be directly computed by using eq. (4.11), since the integral converges. The VEV's of $\varphi_{3}$ contains a logarithm divergence and is calculated in Appendix C. This is the mirror situation encountered in Section 6.3 for the VEV's $\left\langle\varphi_{4}\right\rangle_{3}$ and therefore it can be fixed as in the previous subsection:

$$
\left\langle\varphi_{3}\right\rangle_{4}=\frac{3 \pi}{4 \Delta_{3}} B_{14}\left(\ln \frac{g_{4}}{g_{4}^{0}}+\text { const }\right) .
$$


Finally, by supersymmetry we expect $\left\langle\varphi_{4}\right\rangle_{4}=0$, because the field $\varphi_{4}(x)$ plays the role of the trace of the stress-energy tensor for this deformation and indeed this is in agreement with formula (4.11). The values of the VEV may be found in Table 20.

Concerning the UV properties of the theory, there are two fields which need renormalization. The first is the sub-leading magnetization $\sigma^{\prime}(x)$, which mixes logarithmically with the magnetization field $\sigma(x)$. The second is the vacancy density field $t(x)$, which mixes with the energy density $\epsilon(x)$. To avoid the difficulties related to the mixing induced by renormalization, for this deformation it is convenient to rely only on the Form Factor expansion for estimating the correlation functions. In fact, all fields, except the sub-leading magnetization $\sigma^{\prime}(x)$, turn out to be uniquely identified by their symmetry properties. Moreover, the spectral series based on the Form Factor are also able in this case to capture successfully the ultraviolet behavior of the correlators [56]. Hence, for this deformation the integrals (4.24) will be estimated only in terms of $I_{2}(0)$, i.e. $I \simeq I_{2}(0)$. The two-particle matrix elements on the kink states for the operators $\varphi_{1}, \varphi_{2}$ and $\varphi_{4}$ have been calculated in [56] by using a mapping of the TIM onto the dilute q-state Potts model, with $q=2$. All these operators are coupled to states with zero topological charge, i.e. to those multi-kink states which begin and end to the the same vacuum. There are two types of such two-kink Form Factors, depending whether the vacuum

$$
\begin{aligned}
& F_{0 \pm 1}^{\varphi_{i}}\left(\theta_{1}-\theta_{2}\right) \equiv\left\langle 0_{0}\left|\varphi_{i}\right| K_{0 \pm 1}\left(\theta_{1}\right) K_{ \pm 10}\left(\theta_{2}\right) \mid 0_{0}\right\rangle ; \\
& F_{ \pm 10}^{\varphi_{i}}\left(\theta_{1}-\theta_{2}\right) \equiv\left\langle 0_{ \pm 1}\left|\varphi_{i}\right| K_{ \pm 10}\left(\theta_{1}\right) K_{0 \pm 1}\left(\theta_{2}\right) \mid 0_{ \pm 1}\right\rangle
\end{aligned}
$$

where $\varphi_{i}$ designs the operator under consideration. The Form Factors can be conveniently parameterized as

$$
\begin{array}{ll}
F_{0 \pm 1}^{\varphi_{i}}(\theta)=F_{-}^{\varphi_{i}}(\theta), & i=2,4 \\
F_{ \pm 10}^{\varphi_{i}}(\theta)=F_{+}^{\varphi_{i}}(\theta), & i=2,4 \\
F_{0 \pm 1}^{\varphi_{1}}(\theta)= \pm F_{-}^{\varphi_{1}}(\theta), & \\
F_{ \pm 10}^{\varphi_{1}}(\theta)= \pm F_{+}^{\varphi_{1}}(\theta), &
\end{array}
$$

and their expressions are given by 56]

$$
\begin{aligned}
& F_{ \pm}^{\varphi_{4}}(\theta)=-i \pi m^{2} e^{ \pm \frac{\gamma}{2}(\pi+i \theta)} \frac{\cosh \frac{\theta}{2}}{\sinh \frac{1}{4}(\theta-i \pi)} F_{0}(\theta) \\
& F_{ \pm}^{\varphi_{2}}(\theta)= \pm i\left(\left\langle 0_{ \pm 1}\left|\varphi_{2}\right| 0_{ \pm 1}\right\rangle-\left\langle 0_{0}\left|\varphi_{2}\right| 0_{0}\right\rangle\right) \frac{e^{ \pm \frac{\gamma}{2}(\pi+i \theta)}}{4 \sinh \frac{1}{4}(\theta-i \pi)} F_{0}(\theta) \\
& F_{ \pm}^{\varphi_{1}}(\theta)=\mp \frac{\left\langle 0_{+1}\left|\varphi_{1}\right| 0_{+1}\right\rangle}{2 \Upsilon_{+}(i \pi)} \frac{e^{ \pm \frac{\gamma}{2}(\pi+i \theta)}}{\cosh \frac{\theta}{2}} \Upsilon_{ \pm}(\theta) F_{0}(\theta)
\end{aligned}
$$

\footnotetext{
${ }^{10}$ Note that we use a different notation for the vacua compared to the one used in [40]. These notations enable us to specify more clearly the disorder vacuum $\left|0_{0}\right\rangle$ (with zero magnetization) and the two ordered vacua $\left|0_{ \pm 1}\right\rangle$ (with \pm 1 magnetization).
} 
with

$$
\begin{gathered}
F_{0}(\theta)=-i \sinh \frac{\theta}{2} \exp \left\{\int_{0}^{\infty}-\frac{d x}{x} \frac{\sinh \left(\frac{3 x}{2}\right)}{\sinh 2 x \cosh \frac{x}{2}} \frac{\sin ^{2}(i \pi-\theta) \frac{x}{2 \pi}}{\sinh x}\right\}, \\
\Upsilon_{+}(\theta)=\exp \left\{2 \int_{0}^{\infty} \frac{d x}{x} \frac{\sinh \left(\frac{p}{2}-1\right) x}{\sinh \frac{p x}{2}} \frac{\sin ^{2}(2 i \pi-\theta) \frac{x}{2 \pi}}{\sinh 2 x}\right\} \\
\Upsilon_{-}(\theta)=\Upsilon_{+}(\theta+2 i \pi) \\
\gamma=\frac{1}{2 \pi} \ln 2 .
\end{gathered}
$$

With the knowledge of the first Form Factors, the spectral representations of the correlation functions involving the fields $\varphi_{1}(x), \varphi_{2}(x)$ and $\varphi_{4}(x)$ are approximated by

$$
\begin{gathered}
\left\langle 0_{0}\left|\varphi_{i}(x) \varphi_{j}(0)\right| 0_{0}\right\rangle \simeq \sum_{k= \pm} \int_{\theta_{1}>\theta_{2}} \frac{d \theta_{1}}{2 \pi} \frac{d \theta_{2}}{2 \pi} F_{0 \pm 1}^{\varphi_{i}}\left(\theta_{1}-\theta_{2}\right) F_{0 \pm 1}^{\varphi_{j}}\left(\theta_{2}-\theta_{1}\right) e^{-|x| E_{2}} \\
\left\langle 0_{ \pm 1}\left|\varphi_{i}(x) \varphi_{j}(0)\right| 0_{ \pm 1}\right\rangle \simeq \int_{\theta_{1}>\theta_{2}} \frac{d \theta_{1}}{2 \pi} \frac{d \theta_{2}}{2 \pi} F_{ \pm 10}^{\varphi_{i}}\left(\theta_{1}-\theta_{2}\right) F_{ \pm 10}^{\varphi_{j}}\left(\theta_{2}-\theta_{1}\right) e^{-|x| E_{2}}
\end{gathered}
$$

where $i, j=1,2,4$ and $E_{2}=m\left(\cosh \theta_{1}+\cosh \theta_{2}\right)$ is the energy of the two-kink asymptotic state. By integrating the above expressions, one can obtain the associated amplitudes. The convergence of the above spectral series has been successfully checked against the $\Delta$-theorem sum rule (when this applies) [56]. Our results for the amplitudes are in Table 21.

\section{Conclusions}

In this paper we have exploited some powerful techniques of Quantum Field Theory in order to compute an ample set of universal amplitude ratios for the scaling region of the two-dimensional Tricritical Ising Model. The determination of the thermodynamical amplitudes entering the universal ratios has been obtained by combining exact non-perturbative results coming from CFT (UV theory) and from Scattering Theory (IR theory). More specifically, we have used the ultraviolet data provided by CFT for setting up an Operator Product Expansion (and computing the first order approximation to the structure constants), and for finding eigenvalues and eigenvectors of the off-critical hamiltonian by a numerical approach. In addition, we have used non-perturbative approaches related to the integrability of several deformations of the model to obtain important infrared data, as for instance the exact values of the Vacuum Expectation Values of the order parameters, the exact spectra of the massive excitations and the first Form Factors. A judicious use of the ultraviolet and infrared properties of the various fields which span the scaling region of the model have allowed us to reduce significantly the analytic

efforts necessary to compute the universal ratios. Some of these quantities can be found 
in Tables 22-26 As already pointed out in the text, this large set of quantities may be quite useful for an experimental investigation of the critical properties of this class of universality and we hope that the results presented in this paper may stimulate such experimental activity. It would be equally interesting to extend the theoretical approach discussed here to other two-dimensional models in order to reach a full control of the classes of universalities of low-dimensional systems.

Acknowledgements. We are pleased to thank Michele Caselle, Alexander and Alexei Zamolodchikov for useful discussions. This work has been done under partial support of the EC TMR Programme ERBFMRXCT960012 Integrability, non-perturbative effects and symmetry in Quantum Field Theories. D.F. would like to thank I.N.F.N. for a fellowship and S.I.S.S.A. for hospitality.

\footnotetext{
${ }^{11}$ In the calculation of the universal ratios, we have used the exact values of the susceptibilities when available from the $\Delta$-theorem sum rule, otherwise we have used the arithmetic mean of their determinations obtained by the fluctuation-dissipation theorem and the numerical TCSA.
} 


\section{A Mellin regularization scheme}

The aim of this appendix is to discuss a regularization of the integrals (4.33). They can be written as

$$
C_{i j}^{p(1)}=-\int^{\prime} d^{2} w g(w, \bar{w})
$$

with

$$
g(w, \bar{w}) \equiv\left\langle\tilde{A}^{p}(\infty) \tilde{\Phi}(w) \tilde{\varphi}_{i}(1) \tilde{\varphi}_{j}(0)\right\rangle_{C F T} .
$$

A regularized version of the divergent integrals (4.33) is provided by the following function of the large distance cut-off $R \equiv 1 / a$

$$
I(a)=-\int d^{2} w \Theta(a|w|) g(w, \bar{w})
$$

where the cut-off function $\Theta(t)$ has a fast decreasing behavior at $t \rightarrow+\infty$ to make the integral convergent and is equal to 1 for $t \rightarrow 0^{+}$. Of course $I(a)$ diverges for $a \rightarrow 0^{+}$in a way that depends on the particular choice of $\Theta(t)$, but it converges to a finite value for $a \rightarrow+\infty$ thanks to the fast decreasing behavior of the cut-off function. The finite part of the integral (A.3) is independent of the parameter $a$ and furnishes its natural regularization. It coincides with the analytic continuation of the integral (A.1) from those regions of the conformal weights for which it converges. The finite part of the integral can be explicitly calculated by means of the Mellin transform of the complex function $I(a)$ defined by

$$
\tilde{I}(s)=\int_{0}^{\infty} \frac{d a}{a} a^{s} I(a) .
$$

In fact, when the Mellin transform have simple poles, it provides the asymptotic expansion in powers of $a$ of the function $I(a)$ according to the formula

$$
I(a)=\sum_{i} \operatorname{Res}\left[a^{-s} \tilde{I}(s)\right]_{s=s_{i}}
$$

where the sum runs over the poles. In our cases the above sum is finite since the theory presents only a finite number of IR divergent terms. The finite part of $I(a)$ is therefore given by

$$
I_{0}=\operatorname{Res}[\tilde{I}(s)]_{s=0}=\lim _{s \rightarrow 0} s \tilde{I}(s)
$$

Finally, we also need the following theorem on convolution: if the function $I(a)$ has the form of a convolution (as in equation (A.3)), then its Mellin transform is given by

$$
\tilde{I}(s)=-\tilde{\Theta}(s) \tilde{G}(2-s),
$$

where $\tilde{\Theta}(s)$ is the Mellin transform of $\Theta(t)$ and $\tilde{G}(s)$ may be considered as the Mellin transform of the angular integral of $g(|w|, \arg (w))$ with respect to the radial coordinate $|w|$

$$
\tilde{G}(2-s)=\int d^{2} w|w|^{-s} g(w, \bar{w})
$$


In our calculation we have used for $\Theta(t)$ the function

$$
\Theta(t)=e^{-t},
$$

whose Mellin transformation is the Gamma function

$$
\tilde{\Theta}(s)=\Gamma(s) .
$$

For the calculation of $\tilde{G}(2-s)$ we refer the reader to the next appendix.

\section{B Calculation of the $\left(C_{i j}^{p(1)}\right)_{k}$}

In this appendix, we show how to compute the first correction to the structure constants, i.e. the finite part of

$$
\left(C_{i j}^{p}\right)_{k}^{(1)}=\int^{\prime}\left\langle\varphi_{p}(\infty) \Phi_{k}(w) \varphi_{j}(1) \varphi_{i}(0)\right\rangle d^{2} w .
$$

The conformal four-point correlation functions entering the integral may be computed by means of the modified Coulomb Gas methods [5]. In this approach the central charge $c$ is parameterized by

$$
\begin{array}{ccc}
c=1-24 \alpha_{0}^{2} & ; \quad \alpha_{ \pm}=\alpha_{0} \pm \sqrt{\alpha_{0}^{2}+1} \\
\alpha_{+} \alpha_{-} & =-1 .
\end{array}
$$

The vertex operators are defined by $V_{n m}(x)=: e^{i \alpha_{n m} \Phi(x)}$ : where $\Phi(x)$ is a free scalar field and the charges $\alpha_{n m}$ defined by

$$
\alpha_{n m}=\frac{1}{2}(1-n) \alpha_{-}+\frac{1}{2}(1-m) \alpha_{+} .
$$

The conformal dimension of the operator $V_{n m}(x)$ is given by $\Delta_{n m}=-\overline{\alpha_{n m}} \alpha_{n m}$ with

$$
\overline{\alpha_{n m}}=2 \alpha_{0}-\alpha_{n m}=\frac{1}{2}(1+n) \alpha_{-}+\frac{1}{2}(1+m) \alpha_{+} .
$$

The integrals encountered in the our computation were of two types.

In the first case, no screening charge is needed, therefore they can be computed in a straightforward way. For instance, this is the case of the integral of the 4-point correlation function $\left\langle\varphi_{1}\left(x_{1}\right) \varphi_{2}\left(x_{2}\right) \varphi_{2}\left(x_{3}\right) \varphi_{3}\left(x_{4}\right)\right\rangle$. As an example, we provide the calculation of $\left(C_{22}^{3}\right)_{1}^{(1)}$ in the magnetic deformation of the TIM:

$$
\left(C_{22}^{3(1)}\right)_{1}=-\int^{\prime}\left\langle\varphi_{3}(\infty) \varphi_{2}(1) \varphi_{1}(z) \varphi_{2}(0)\right\rangle .
$$


Since $2 \alpha_{12}+\alpha_{22}+\alpha_{21}=2 \alpha_{0}$, no screening charge is needed. Therefore,

$$
\left(C_{22}^{3(1)}\right)_{1}=N \int d^{2} z|z|^{-\frac{1}{5}}|z-1|^{-\frac{1}{5}}
$$

where $N$ is a normalization factor which is fixed by the operator algebra and the structure constants (see Table 2):

$$
N=c_{4} c_{5}=\frac{1}{2} \sqrt{\frac{\Gamma\left(\frac{4}{5}\right) \Gamma^{3}\left(\frac{4}{5}\right)}{\Gamma\left(\frac{1}{5}\right) \Gamma^{3}\left(\frac{3}{5}\right)}}
$$

Using

$$
\int d^{2} z|z|^{2 a}|z-1|^{2 b}=-S(b) B(1+a, 1+b) B(1+b,-1-a-b),
$$

with

$$
B(\alpha, \beta)=\frac{\Gamma(\alpha) \Gamma(\beta)}{\Gamma(\alpha+\beta)} \quad, \quad S(x) \equiv \sin \pi x
$$

we find:

$$
\left(C_{22}^{3(1)}\right)_{1}=-\frac{25}{32} \frac{S^{2}\left(\frac{1}{10}\right) S^{\frac{1}{2}}\left(\frac{1}{5}\right)}{S^{\frac{3}{2}}\left(\frac{2}{5}\right)} \frac{\Gamma^{4}\left(\frac{9}{10}\right) \Gamma\left(\frac{1}{5}\right)}{\Gamma^{3}\left(\frac{3}{5}\right)} .
$$

In the second case, one screening operator is needed and the integral takes the following form:

$$
Z(a, b, c, d, e)=\int d^{2} w \int d^{2} z|z|^{2 a}|1-z|^{2 b}|w-z|^{2 c}|w|^{2 d}|1-w|^{2 e} .
$$

The exponents $a, b, c, d, e$ are computed in the Coulomb gas formalism [5]. Notice that the exponent $d$ is in fact $d^{\prime}-s / 2$, where $s$ comes from the Mellin regularization scheme (see Appendix A). It can be shown using transformations in the complex plane that [5]

$$
\begin{aligned}
Z(a, b, c, d, e)= & S(a+c)^{-1} \int d^{2} w|w|^{2 d}|1-w|^{2 e} \times \\
& {\left[S(a+b+c) S(b)\left|I_{1}(a, b, c ; w)\right|^{2}+S(a) S(c)\left|I_{2}(a, b, c ; w)\right|^{2}\right], }
\end{aligned}
$$

where

$$
\begin{aligned}
& I_{1}(a, b, c ; \eta) \equiv \frac{\Gamma(-a-b-c-1) \Gamma(b+1)}{\Gamma(-a-c)}{ }_{2} F_{1}(-c,-a-b-c-1 ;-a-c ; \eta) \\
& I_{2}(a, b, c ; \eta) \equiv \eta^{1+a+c} \frac{\Gamma(a+1) \Gamma(c+1)}{\Gamma(a+c+2)}{ }_{2} F_{1}(-b, a+1 ; a+c+2 ; \eta)
\end{aligned}
$$

The generalized hypergeometric functions are defined by

$$
{ }_{p} F_{q}\left(a_{1}, \cdots, a_{p} ; b_{1},, \cdots, b_{q} ; z\right) \equiv \sum_{k=0}^{\infty} \frac{\left(a_{1}\right)_{k} \cdots\left(a_{p}\right)_{k}}{k !\left(b_{1}\right)_{k} \cdots\left(b_{q}\right)_{k}} z^{k},
$$

with $(a)_{k} \equiv \Gamma(a+k) / \Gamma(a)$.

There are several ways to compute $Z(a, b, c, d, e)$. We have used two equivalent methods in order to have a non-trivial check. Let us explain briefly them for completeness. 
- The first method is the one considered in [44]. Using monodromy properties of the integral, we can write $Z$ as :

$$
Z=z_{11} J_{1}^{2}+z_{22} J_{2}^{2}+z_{12} J_{1} J_{2}
$$

with

$$
\begin{gathered}
J_{1} \equiv \int_{0}^{1} z^{d}(1-z)^{e} I_{1}(a, b, c, z)=B(b+1,-a-b-c-1) B(d+1, e+1) \\
\quad \times{ }_{3} F_{2}(-c,-a-b-c-1, d+1 ;-a-c, 2+d+e ; 1),
\end{gathered}
$$

and

$$
\begin{aligned}
J_{2} \equiv & \int_{0}^{1} z^{d}(1-z)^{e} I_{2}(a, b, c, z)=B(a+1, c+1) B(2+a+c+d, e+1) \\
& \times{ }_{3} F_{2}(-b, a+1,2+a+c+d ; a+c+2,3+a+c+d+e ; 1) .
\end{aligned}
$$

In these formulae the $z_{i j}$ are defined by:

$$
\begin{aligned}
z_{11}= & -\frac{1}{4} S(a+c)^{-2} S^{-1}(c+d+e) S^{-1}(a+b+c+d+e) \times \\
& S(b) S(a+b+c) S(d) \times \\
& (S(b-c-d)-S(b+c-d)+S(b+c+d)-S(2 a+b+c+d) \\
& -S(b+c+d+2 e)+S(2 a+b+3 c+d+2 e)) \\
z_{22}= & \frac{1}{4} S(a+c)^{-2} S^{-1}(c+d+e) S^{-1}(a+b+c+d+e) \times \\
& S(a) S(c) S(a+c+d) \times \\
& (S(a+b-d)+S(a-b+d)-S(a+b+d)+S(a+b+2 c+d) \\
& -S(a-b-d-2 e)-S(a+b+2 c+d+2 e)) \\
z_{12}= & 2 S(a+c)^{-2} S^{-1}(c+d+e) S^{-1}(a+b+c+d+e) \times \\
& S(a) S(b) S(c) S(a+b+c) S(d) S(a+c+d)
\end{aligned}
$$

- The second method is the one considered in [57]. It consists in decomposing $Z$ into its holomorphic and antiholomorphic parts:

$$
I=s(b) s(e)\left[J_{1}^{+} J_{1}^{-}+J_{2}^{+} J_{2}^{-}\right]+s(b) s(e+c) J_{1}^{+} J_{2}^{-}+s(b+c) s(e) J_{2}^{+} J_{1}^{-},
$$

where

$$
\begin{aligned}
& J_{1}^{+}=J(a, b, c, d, e) ; J_{2}^{+}=J(b, a, c, e, d) ; \\
& J_{1}^{-}=J(b,-2-a-b-c, c, e,-2-d-e-c) ; \\
& J_{2}^{-}=J(-2-a-b-c, b, c,-2-d-e-c, e),
\end{aligned}
$$


with the notation

$$
\begin{aligned}
& J(a, b, c, d, e)=\int_{0}^{1} d u \int_{0}^{1} d v u^{a+d+c+1}(1-u)^{b} v^{d}(1-v)^{c}(1-u v)^{e}=B(1+c, 1+d) \times \\
& \times B(2+a+d+c, 1+b)_{3} F_{2}(-e, 2+c+d, 1+d, 3+a+b+c+d, 2+c+d ; 1) .
\end{aligned}
$$

The $J$ integrals appearing in (B.24) are not independent. Using a contour deformation it can be shown that they are related as

$$
\begin{aligned}
& S(a+b+c) J_{1}^{-}+S(a+b) J_{2}^{-}=\frac{S(a)}{S(c+d+e)}\left(S(d) J_{1}^{+}+S(c+d) J_{2}^{+}\right) \\
& S(c+d+e) J_{2}^{-}+S(d+e) J_{1}^{-}=\frac{S(d)}{S(a+b+c)}\left(S(a) J_{2}^{+}+S(a+c) J_{1}^{+}\right) .
\end{aligned}
$$

This has the advantage that some of the $J_{i}^{ \pm}$can be computed in an easier way than others.

In most cases, we were able to compute exactly these types of integrals in terms of a product of Gamma functions by using some known relations of hypergeometric functions at argument $z=1$ [58]. Given their cumbersome expressions, the results are not reported here. When no close forms were available, the integrals were determined numerically (using the two different representations above) by directly calculating the hypergeometric functions ${ }_{3} F_{2}(z)$ at $z=1$. In this case, we used fast convergent expressions of the hypergeometric functions in order to reach rather accurate results (with approximately $0.5 \%$ of confidence).

We have performed the above set of calculations for the magnetic, thermal and submagnetic perturbations. The results are in Tables 7, 13 and 18. We did not pursue this calculation for the vacancy density perturbation for the UV difficulties explained in the text and also because in this case Form Factors were expected to provide a reasonable approximation of the correlators in all range of $r$.

\section{Regularization of the VEV's.}

The VEV's of primary operators $\Phi_{l, k}$ in the minimal models $\mathcal{M}_{p, p^{\prime}}$ perturbed by an integrable relevant operator $\phi_{i}$ have been conjectured in 40. They can be written as

$$
\left\langle 0_{s}\left|\Phi_{l, k}\right| 0_{s}\right\rangle=B_{(l, k), i, s}(\xi, \eta) g^{\frac{\Delta_{l, k}}{1-\Delta_{i}}}
$$

where $s$ labels the different vacua. The prefactor $B_{(l, k), i, s}(\xi, \eta)$ can be further decomposed as

$$
B_{(l, k), i, s}(\xi, \eta)=b_{(l, k), i, s}(\xi) Q_{i}(\xi, \eta)
$$


where $b_{(l, k), i, s}(\xi)$ is a simple function for any perturbation $\phi_{i}$ and any vacuum $\left|0_{s}\right\rangle$ whereas $Q_{i}(\xi, \eta)$ can be expressed as

$$
Q_{i}(\xi, \eta)=e^{I_{i}(\xi, \eta)} \quad, \quad I_{i}(\xi, \eta)=\int_{0}^{+\infty} d t F_{i}(t ; \xi, \eta)
$$

In Section 4 we have presented the explicit formulae for the three integrable deformations of the TIM. The above integral may diverge for some values of the two parameters

$$
\xi=\frac{p}{p^{\prime}-p} \quad, \quad \eta=(\xi+1) l-\xi k
$$

In fact, due to the asymptotic behavior of $F_{i}(t ; \xi, \eta)$

$$
F_{i}(t ; \xi, \eta) \sim e^{-a_{i}(\xi, \eta) t} \quad, \quad t \rightarrow+\infty
$$

this occurs when $a_{i}(\xi, \eta) \leq 0$. In this case, the integral $I_{i}(\xi, \eta)$ needs to be regularized in order to extract its physical value. Its regularization may be performed in a number of equivalent ways, for instance by means of an analytic prolongation in $\xi$ (from values for which $Q_{i}(\xi, \eta)$ converges), possibly also using a sufficient number of times the reflection equations satisfied by the VEV. Here we present another simple method of controlling the divergences of $I_{i}(\xi, \eta)$, specializing our discussion to the $\operatorname{VEV}\left\langle\Phi_{2,1}\right\rangle_{4}$ of the sub-leading magnetization operator of the TIM in the massive phase reached by the perturbation of the vacancy operator $\phi_{1,3} \equiv t \equiv \varphi_{4}$, with $g_{4}<0$. This example, somehow, presents all possible types of divergences of the above integrals. Let us consider initially the general expression of the function $F$ in the case of $\phi_{1,3}$ perturbation 40]

$$
F_{1,3}(t ; \xi, \eta)=\frac{1}{t}\left(\frac{\cosh 2 t \sinh (\eta-1) t}{2 \cosh t \sinh (\xi t) \sinh (\xi+1) t}-\frac{\eta^{2}-1}{2 \xi(\xi+1)} e^{-4 t}\right)
$$

In our example $\xi=4$ and $\eta=6$, hence

$$
I_{4}(4,6)=\int_{0}^{+\infty} d t F_{4}(t ; 4,6) \quad, \quad F_{4}(t ; 4,6)=\frac{1}{t}\left(\frac{\cosh 2 t \sinh 7 t}{2 \cosh t \sinh 4 t}-\frac{7}{8} e^{-4 t}\right)
$$

and the asymptotic behavior

$$
F_{4}(t ; 4,6) \sim e^{4 t} \quad, \quad t \rightarrow+\infty
$$

leads to the divergence of the above integral. The complete VEV under investigation can be expressed as

$$
\left\langle 0_{s}\left|\sigma^{\prime}\right| 0_{s}\right\rangle=\frac{\sin \frac{3}{2} \pi s}{\sin \frac{\pi}{4} s}\left(\frac{5}{2}\right)^{\frac{7}{8}}\left[\pi \frac{21}{25}\left|g_{4}\right| \sqrt{\frac{\Gamma\left(\frac{1}{5}\right) \Gamma\left(-\frac{7}{5}\right)}{\Gamma\left(\frac{4}{5}\right) \Gamma\left(\frac{12}{5}\right)}}\right]^{\frac{35}{32}} Q_{4}(4,6)
$$


where $s=1,2,3$ labels the three different vacua. Assuming a regularization of $Q_{4}(4,6)$, notice that for $s=2$ the first term in (C.36) implies

$$
\left\langle 0_{s}\left|\sigma^{\prime}\right| 0_{s}\right\rangle=0
$$

To compute $I_{4}(4,6)$, let us break the integral as

$$
I_{4}(4,6)=\int_{0}^{1} d t F_{4}(t ; 4,6)+\int_{1}^{+\infty} d t F_{4}(t ; 4,6) .
$$

The first integral is always convergent since, in general

$$
\lim _{t \rightarrow 0} F_{1,3}(t ; \xi, \eta)=\text { const }
$$

thanks to a compensation between the two terms in C.33). Hence it is sufficient to make an analytic prolongation of the second integral by subtracting its divergent part. This can be done by expressing initially the second integral as

$$
\int_{1}^{+\infty} d t F_{4}(t ; 4,6)=\int_{1}^{+\infty}\left(F_{4}(t ; 4,6)-\frac{e^{4 t}}{2 t}+\frac{e^{2 t}}{2 t}\right) d t+Y(-4)-Y(-2),
$$

and then by making an analytic prolongation of the function

$$
Y(a)=\int_{1}^{+\infty} \frac{d t}{t} e^{-a t} \quad, \quad \Re e(a)>0
$$

to the domain $\Re e(a) \leq 0$. Notice that although the analytic extension of the function $Y(a)$ is not monodromic ( $a=0$ is in fact a branch-cut point), its exponential $e^{Y(a)}$ is however uniquely defined and this is precisely the expression which enters the formula (C.36) for the VEV. In the punctured complex plane $a \in \mathbb{C}-\{0\}$ we have

$$
e^{Y(a)}=\frac{e^{\gamma}}{a} e^{-f(a)}
$$

where $\gamma=0.577216 \ldots$ is the Euler-Mascheroni constant and the holomorphic function $f(a)$ is defined by the power series

$$
f(a)=\sum_{n=1}^{\infty}(-1)^{n} \frac{a^{n}}{n ! n} \quad, \quad a \in \mathbb{C} .
$$

The last series is fastly convergent and allows good numerical estimations.

The divergences which appear in the expression of the VEV proposed in ref. 40] can be generally tamed as in eq. (C.40) above, i.e. by subtracting the leading (and subleading) exponential terms and then performing the analytic continuation of $Y(a)$. However, sometimes the first term in the r.h.s. of (C.40), i.e. the integral accompanied by the subtractions, is still logarithmically divergent. This is in particular the case of our example of $\left\langle\varphi_{3}\right\rangle_{4}$. When this happens, the pure power law behavior in the coupling constant 
$g_{i}$ of the VEV (C.28) gets modified. To face this situation, one may perform an extra regularization of the integral by making a shift of the parameter $\xi$ which characterizes the minimal models

$$
\xi \rightarrow \xi+\epsilon
$$

and then carefully taking the limit $\epsilon \rightarrow 0$. In our example this results in shifting $4 \rightarrow 4+\epsilon$, $6 \rightarrow 6+\epsilon$ and considering the expression

$$
\int_{1}^{+\infty}\left(F_{4}(t ; 4+\epsilon, 6+\epsilon)-\frac{e^{4 t}}{2 t}+\frac{e^{2 t}}{2 t}-\frac{e^{-\epsilon t}}{t}\right) d t+Y(-4)-Y(-2)+Y(\epsilon) .
$$

The limit $\epsilon \rightarrow 0$ of the first integral in the previous equation is obtained by putting $\epsilon=0$ and hence it can be calculated by performing a numerical integration. Concerning the last term, once used to evaluate the VEV, we have

$$
e^{Y(\epsilon)}=\frac{e^{\gamma}}{\epsilon}+\mathcal{O}(1)
$$

Let us consider now the dependence of the VEV on the coupling constant $g \equiv-g_{4}$ once the shift (C.44) has been performed. We have

$$
g^{\frac{35}{32}} \rightarrow g^{\frac{35}{32}+\frac{13}{128} \epsilon}
$$

since the conformal weights depend on $\xi$. By expanding this formula in powers of $\epsilon$ we have

$$
g^{\frac{35}{32}+\frac{13}{128} \epsilon}=\left(g_{0}\right)^{\frac{13}{128} \epsilon} g^{\frac{35}{32}}\left(\frac{g}{g_{0}}\right)^{\frac{13}{128} \epsilon}=g^{\frac{35}{32}}\left[1+\frac{13}{128} \epsilon \ln \frac{g}{g_{0}}+\mathcal{O}\left(\epsilon^{2}\right)\right],
$$

where we have introduced an arbitrary value of the coupling constant $g_{0}$ for taking the logarithm of the adimensional quantity $\frac{g}{g_{0}}$. Once this expression is multiplied with eq. (C.46), it gives rise to

$$
g^{\frac{35}{32}}\left(\frac{e^{\gamma}}{\epsilon}+\frac{13}{128} e^{\gamma} \ln \frac{g}{g_{0}}+\mathcal{O}(1)\right) .
$$

In conclusion, in the example of the VEV ( C.36), apart from a divergence $\frac{1}{\epsilon}$ which can be discarded, it presents a logarithmic part and a power-law term:

$$
\left\langle 0_{s}\left|\sigma^{\prime}\right| 0_{s}\right\rangle=B_{(2,1), 4, s} g^{\frac{35}{32}} \ln \frac{g}{g_{0}}+C_{(2,1), 4, s} g^{\frac{35}{32}} .
$$

It is important to notice that in the previous expression the constant $B_{(2,1), 4, s}$ is uniquely determined by this procedure and is not affected by a change of the reference coupling constant $g_{0}$. Instead, the second constant $C_{(2,1), 4, s}$ may be freely modified by rescaling the arbitrary value of the reference coupling constant $g_{0}$. In this sense, the constant $B_{(2,1), 4, s}$ possesses a precise physical value in QFT and it may enter the definitions of amplitude ratios as $B$ prefactor. 


\section{Form Factors in the thermal sector}

In this appendix, we will discuss some features of the Form Factors in the thermal deformation of the TIM. In particular, we will show that it is necessary to determine independently (for instance by a numerical method) the one-particle Form Factors of the $\varphi_{1}$ in order to compute its higher particle Form Factors. We will discuss these matrix elements in the high-temperature phase of the model with the mass spectrum and the $Z_{2}$ quantum number of the particles given in Table 9. Let

$$
F_{\alpha_{1}, \ldots, \alpha_{n}}\left(\theta_{1}, \ldots, \theta_{n}\right)=\left\langle 0|\sigma(0)| A_{\alpha_{1}}\left(\theta_{1} \ldots A_{\alpha_{n}}\left(\theta_{n}\right)\right\rangle .\right.
$$

Since $\sigma(x)$ is a $Z_{2}$ odd operator, the non-vanishing matrix elements will be those on $Z_{2}$ odd multi-particle states. Hence, the matrix elements which contribute to the summation (4.36) are, in increasing order of total energy of the corresponding states: $F_{1}, F_{3}, F_{12}, F_{6}$, $F_{14}, F_{111}, F_{23}, F_{15}, \ldots$. According to the analysis of ref. [10, 47], the two-particle Form Factors can be conveniently written as

$$
F_{a b}^{\sigma}(\theta)=F_{a b}^{\min }(\theta) \frac{\mathcal{Q}_{a b}^{\sigma}}{D_{a b}},
$$

where $F_{a b}^{\min }$ is the minimal solution of the set of Watson equations which has neither poles neither zero and $\mathcal{Q}_{a b}^{\sigma}$ and $D_{a b}$ are polynomials in $\cosh \theta$. The latter takes into account the pole structure of this matrix element (independent of the field) whereas the former depends on the field under consideration, in this case $\sigma(x)$. To determine the above quantities we need initially the expression of the elastic two-particle $S$-matrix of the model [21, 22] that can be expressed as

$$
S_{a b}(\theta)=\prod_{\alpha \in \mathcal{A}_{a b}} f_{\alpha}(\theta)^{p_{\alpha}},
$$

where

$$
f_{\alpha}(\theta) \equiv \frac{\tanh \frac{1}{2}(\theta+i \pi \alpha)}{\tanh \frac{1}{2}(\theta-i \pi \alpha)} .
$$

The different amplitudes can be found in Table 2 of ref. [47] and are not reported here. The exponents $p_{\alpha}$ denote the multiplicities of the corresponding poles (located at $\theta=i \pi \alpha$ and $\theta=i \pi(1-\alpha)$ ) identified by the indices $\alpha$. Correspondingly, $F_{a b}^{m i n}$ is parameterized by:

$$
F_{a b}^{\min }(\theta)=\left(-i \sinh \frac{\theta}{2}\right)^{\delta_{a, b}} \prod_{\alpha \in \mathcal{A}_{a b}} g_{\alpha}(\theta)^{p_{\alpha}}
$$

where $g_{\alpha}(\theta)$ is given by the integral representation

$$
g_{\alpha}(\theta)=\exp \left[2 \int_{0}^{\infty} \frac{d t}{t} \frac{\cosh [(\alpha-1 / 2) t]}{\cosh t / 2 \sinh t} \sin ^{2}(\hat{\theta} t / 2 \pi)\right],
$$


with $\hat{\theta}=i \pi-\theta$. The polynomials $D_{a b}(\theta)$ are entirely determined from the poles of the $S-$ matrix. According to [10], they are given by

$$
\begin{aligned}
& D_{a b}(\theta)=\prod_{\alpha \in \mathcal{A}_{a b}}\left(\mathcal{P}_{\alpha}(\theta)\right)^{i_{\alpha}}\left(\mathcal{P}_{1-\alpha}(\theta)\right)^{j_{\alpha}}, \\
& i_{\alpha}=n+1, \quad j_{\alpha}=n, \text { if } \quad p_{\alpha}=2 n+1 ; \\
& i_{\alpha}=n, \quad j_{\alpha}=n \text {, if } \quad p_{\alpha}=2 n \text {, }
\end{aligned}
$$

where $\mathcal{A}_{a b}$ and $p_{\alpha}$ are defined in eq. (D.53) and can be read from Table 2 of ref. [47]. The functions

$$
\mathcal{P}_{\alpha}(\theta) \equiv \frac{\cos \pi \alpha-\cosh \theta}{2 \cos ^{2} \frac{\pi \alpha}{2}},
$$

give a suitable parameterization of the pole of the $\mathrm{FF}$ at $\theta=i \pi \alpha$. The asymptotic behavior of $g_{\alpha}(\theta)$ and $\mathcal{P}_{\alpha}(\theta)$ is given by

$$
g_{\alpha}(\theta) \sim e^{\frac{|\theta|}{2}} \quad ; \quad \mathcal{P}_{\alpha}(\theta) \sim e^{|\theta|} \quad \text { for } \theta \rightarrow \infty .
$$

An upper bound on the maximal degree of the polynomial $\mathcal{Q}_{a b}^{\sigma}$ can be fixed by the constraint [10

$$
y \leq \Delta_{\sigma}
$$

where $y$ is defined by

$$
\lim _{\left|\theta_{i}\right| \rightarrow \infty} F_{a_{1}, \ldots, a_{n}}\left(\theta_{1}, \ldots, \theta_{n}\right) \sim e^{y\left|\theta_{i}\right|} .
$$

Collecting all the above results, let us consider the two-particle Form Factor $F_{12}^{\sigma}(\theta)$ :

$$
F_{12}^{\sigma}(\theta)=F_{12}^{\min }(\theta) \frac{\mathcal{Q}_{12}^{\sigma}}{D_{12}(\theta)} .
$$

By using eqs. (D.61) and (D.62), for the degree $\delta$ of $\mathcal{Q}_{12}$ we have $\delta \leq 1$. The residue equations (4.42) at the simple order poles corresponding to the bound states supply us with two equations, namely

$$
-i \lim _{\theta \rightarrow i u_{a b}^{c}}\left(\theta-i u_{a b}^{c}\right) F_{a b}^{\sigma}(\theta)=\gamma_{a b}^{c} F_{c}^{\sigma}
$$

with $a=1, b=2, c=1,3$ and $\gamma_{a b}^{c}$ is given by

$$
-i \lim _{\theta \rightarrow i u_{a b}^{c}}\left(\theta-i u_{a b}^{c}\right) S_{a b}(\theta)=\left(\gamma_{a b}^{c}\right)^{2} .
$$

These two equations are able to fix unambiguously $F_{12}^{\sigma}$ provided $F_{1}^{\sigma}$ and $F_{3}^{\sigma}$ are known. However, there is no way to determine these one-particle Form Factors in the bootstrap program. Notice, in fact, that the above equations are also satisfied by the two-particle FF of the subleading magnetization $\sigma^{\prime}(x)$. Therefore, one needs to extract the one-particle FF of $\sigma(x)$ and $\sigma^{\prime}(x)$ by means of some other independent method, as for instance the one provided by the numerical Truncated Conformal Space Approach, discussed in Section 5. 


\section{References}

[1] M.E. Fisher, Phys. Rev. Lett. 66 (1966), 11; R.B. Griffith, Phys. Rev. Lett. 24 (1970), 1479.

[2] A.A. Belavin, A.M. Polyakov, A.B. Zamolodchikov, Nucl.Phys. B 241 (1984), 333

[3] D. Friedan, Z. Qiu, S. Shenker, Phys. Rev. Lett. 52 (1984), 1575

[4] P. Di Francesco, P. Mathieu and D. Senechal, Conformal Field Theory, SpringerVerlag (New York) 1997.

[5] Vl.S. Dotsenko and V.A. Fateev, Nucl. Phys. B 240 [FS 12] (1984), 312; Nucl. Phys. B 251, 691 (1985).

[6] V. Privman, P.C. Hohenberg and A. Aharony, Universal Critical-Point Amplitude Relations, in Phase Transitions and Critical Phenomena, edited by C. Domb and J.L. Lebowitz (Academic Press Limited, London, 1991), Vol. 14.

[7] C. Bervillier, Phys. Rev. B 14 (1976), 4964; D. Stauffer, M. Ferer and M. Wortis, Phys. Rev. Lett. 29 (1972), 345.

[8] J.L. Cardy and G. Mussardo, Nucl. Phys. B 410 (1993), 451.

[9] A.B. Zamolodchikov, Int. J. Mod. Phys. A 3 (1988), 743.

[10] G. Delfino and G. Mussardo, Nucl. Phys. B 455 (1995), 724.

[11] G. Delfino and P. Simonetti Phys. Lett. B 383 (1996), 450; M. Caselle and M. Hasenbusch, Nucl. Phys. B 579 (2000); M. Caselle, P. Grinza and N. Magnoli, Nucl. Phys. B 579 (2000), 635.

[12] G. Delfino, Phys. Lett. B 419 (1998), 291.

[13] J. Salas and A.D. Sokal, J. Stat. Phys. 98 (2000).

[14] R. Guida and N. Magnoli, Nucl. Phys. B 483 (1997), 563.

[15] J.L. Cardy, Journ. Phys. A 25 (1992), L201.

[16] G. Delfino and J.L. Cardy, Nucl. Phys. B 519 (1998), 551; G. Delfino, G.T. Barkema, J.L. Cardy, Nucl.Phys. B 565 (2000) 521; G. Delfino and J. Cardy, Phys. Lett. B 483 (2000), 303.

[17] M. Caselle, R. Tateo and S. Vinti, Nucl. Phys. B 562 (1999), 549. 
[18] D. Fioravanti, G. Mussardo and P. Simon, Phys. Rev. Lett. 85 (2000), 126.

[19] A.B. Zamolodchikov, Sov. J. Nucl. Phys. 44 (1986), 529.

[20] T. Eguchi and S.K. Yang, Phys. Lett. B 224 (1989), 373.

[21] P. Christe, G. Mussardo, Nucl. Phys. B 330 (1990), 465.

[22] V.A. Fateev, A.B. Zamolodchikov, Int. J. Mod. Phys. A 5 (1990), 1025.

[23] D.A. Kastor, E.J. Martinec, S.H. Shenker, Nucl. Phys. B 316 (1989), 590

[24] Al.B. Zamolodchikov, Nucl. Phys. B 358 (1991), 524.

[25] G. Delfino, G. Mussardo and P. Simonetti, Phys. Rev. D 51 (1995), 6620.

[26] A.B. Zamolodchikov, in Beijing 1989, Proceedings Fields, strings and quantum gravity 349. Al.B. Zamolodchikov, Nucl. Phys. B 358 (1991), 497.

[27] M. Lässig, G. Mussardo and J.L. Cardy, Nucl. Phys. B 348 (1991), 591.

[28] F.A. Smirnov, Int. J. Mod. Phys. A 6 (1991), 1407.

[29] F. Colomo, A. Koubek and G. Mussardo, Int. J. Mod. Phys. A 7 (1992), 5281.

[30] I.D. Lawrie, S. Sarbach, in Phase Transitions and Critical Phenomena, vol. 9, C. Domb and J. Lebowitz (Eds.), New York (Academic Press 1984)

[31] M. Blume, Phys. Rev. 141 (1966), 517; H.W. Capel, Physica 32 (1966), 866; R.B. Griffiths, Physica 33 (1967), 689; M. Blume, V.J. Emery, R.B. Griffiths, Phys. Rev. A 4 (1971), 1071; M.J.Tejwani, O. Ferreira, O.E. Vilches, Phys. Rev. Lett. 44 (1980), 152

[32] S.O. Warnaar, B. Nienhuis and K.A. Seaton, Phys. Rev. Lett. 69 (1992), 710; S.O. Warnaar and P.A. Pearce, Int. J. Mod. Phys. A 11 (1996), 291; M.T. Batchelor and K.A. Seaton, Eur. Phys. J. B 5 (1998), 719; J. Suzuki, hep-th/9911216.

[33] D. Friedan, Z. Qiu, S. Shenker, Phys. Lett. B 151 (1984), 1575

[34] Z. Qiu, Nucl. Phys. B 270 (1986), 205.

[35] G. Mussardo, G. Sotkov and M. Stanishkov Nucl. Phys. B 305 (1988), 69.

[36] Al.B. Zamolodchikov, Nucl. Phys. B 342 (1990), 695.

[37] V.A. Fateev, Phys. Lett. B 324 (1994), 45. 
[38] V.P. Yurov and Al.B. Zamolodchikov, Int. J. Mod. Phys. A 6 (1991), 4557.

[39] S.Lukyanov and A.B. Zamolodchikov, Nucl. Phys. B 493 (1997), 571.

[40] V. Fateev, S. Lukyanov, A.B. Zamolodchikov, Al.B. Zamolodchikov, Nucl. Phys. B 516 (1998), 652.

[41] R. Guida and N. Magnoli, Phys.Lett. B 411 (1997) 127.

[42] A.B. Zamolodchikov, JEPT Lett. 43 (1986), 730; J.L. Cardy, Phys. Rev. Lett. 60 (1988), 2709.

[43] G. Delfino, P. Simonetti and J.L. Cardy, Phys. Lett. B 387 (1996), 327.

[44] R. Guida and N. Magnoli, Int. J. Mod. Phys. A 13 (1998), 1145.

[45] Al.B. Zamolodchikov, Nucl. Phys. B 348 (1991), 619.

[46] G. Delfino and G. Mussardo, Phys. Lett. B 324 (1994) 40.

[47] C. Acerbi, G. Mussardo and A. Valleriani, Int. J. Mod. Phys. A 11 (1996) 5327.

[48] K.M. Watson, Phys. Rev. 95 (1954), 228.

[49] M. Karowski, P. Weisz, Nucl. Phys. B 139 (1978), 445.

[50] F.A. Smirnov, Form Factors in Completely Integrable Models of Quantum Field Theory (World Scientific), 1992 and references therein.

[51] J. L. Cardy, Nucl. Phys. B 270 [FS16] (1986) 186; Nucl. Phys. B 275 [FS17] (1986) 200.

[52] M. Lässig and G. Mussardo, Comp. Phys. Comm. 66 (1991) 71.

[53] A. LeClair and G. Mussardo, Nucl. Phys. B 552 (1999), 624.

[54] A. Koubek and G. Mussardo, Phys. Lett. B 311 (1993), 193.

[55] C. Acerbi, G. Mussardo and A. Valleriani, J. Phys. A 30 (1997), 2895.

[56] G. Delfino, Nucl. Phys. B 554 (1999) 537.

[57] Vl. S. Dotsenko, M. Picco and P. Pujol, Nucl. Phys. B 455, (1995), 701.

[58] A. P. Prudnikov, Yu. A. Brychov, O. I. Marichev, Integral and Series Vol. 3, Gordon Breach Science Publishers, 1990. 


\section{Table Caption}

Table 1 . Kac table of the Tricritical Ising Model.

Table 2 . Fusion Rules and structure constants of the TIM for the scalar fields.

Table 3 . Operator content and LG fields.

Table 4 . Nature of QFT's for each individual deformation of the TIM.

Table 5 . Numerical VEV $B_{i j}$ of the four relevant operators of the TIM perturbed by the magnetic $\left(\varphi_{1}\right)$ operator.

Table 6 . Numerical estimation of the one-FF in the magnetic deformation in units of $g_{1}^{\frac{\Delta_{i}}{1-\Delta_{1}}}$.

Table 7 . Numerical values of the first correction of the structure constants $\left(C_{i j}^{k}\right)_{1}^{(1)}=$ $-\int^{\prime} d^{2} z\left\langle\varphi_{k}(\infty) \varphi_{j}(1) \varphi_{1}(z) \varphi_{i}(0)\right\rangle$ where $1 \leq i, j \leq 4$ and $0 \leq k \leq 4$ (with the definition $\varphi_{0} \equiv I, I$ the identity operator). The note ' 2 screening ops.' means that the calculation would have required two screening operators in the Coulomb gas integral.

Table 8 . Estimated amplitudes in the magnetic deformation. The number with ${ }^{\dagger}$ refers to the amplitude in front of the logarithm. The number with ${ }^{*}$ refers to the finite part of the susceptibility.

Table 9 . Mass spectrum in the high-temperature phase.

Table 10 . Numerical and exact (when available) VEV $B_{i 2}$ of the four relevant operators of the TIM perturbed by the thermal $\left(\varphi_{2}\right)$ operator.

Table 11 . Numerical estimation of the first four one-particle FF in the high temperature phase in units of $g_{2}^{\frac{\Delta_{i}}{1-\Delta_{2}}}$. Some of them vanish in virtue of the $Z_{2}$ spin reversal symmetry.

Table 12 . Numerical estimation of the first two one-particle FF in the low temperature phase in units of $\left|g_{2}\right|^{\frac{\Delta_{i}}{1-\Delta_{2}}}$.

Table 13 . Numerical values of the first correction of the structure constants $\left(C_{i j}^{k}\right)_{2}^{(1)}=$ $-\int^{\prime} d^{2} z\left\langle\varphi_{k}(\infty) \varphi_{j}(1) \varphi_{2}(z) \varphi_{i}(0)\right\rangle$ where $1 \leq i, j \leq 4$ and $0 \leq k \leq 4$ (with the definition $\varphi_{0} \equiv I, I$ the identity operator $)$. 
Table 14 . Estimated amplitudes in the high temperature phase. The number with * refers to the finite part of the susceptibility.

Table 15 . Estimated amplitudes in the low temperature phase. The number with ${ }^{\dagger}$ refers to the exact amplitude in front of the logarithm. The number with * refers to the finite part of the susceptibility.

Table 16 . Exact VEV of the four relevant operators in $\sigma^{\prime}$ deformation (from [40]). The numbers with ${ }^{\dagger}$ refer to the amplitudes in front of the logarithm.

Table 17 . Numerical estimation of some one-particle FF in the subleading magnetic perturbation in units of $g_{3}^{\frac{\Delta_{i}}{1-\Delta_{3}}}$. Those relative to $\varphi_{3}$ and $\varphi_{4}$ are not accessible.

Table 18 . Numerical values of the first correction of some structure constants $\left(C_{i j}^{k}\right)_{3}^{(1)}$ with $1 \leq i, j \leq 4$ and $0 \leq k \leq 4$.

Table 19 . Estimated amplitudes in the subleading magnetization deformation for both vacua (the subscripts $a$ and $b$ are respectively for $\left|0_{2}\right\rangle$ and $\left|0_{4}\right\rangle$ ) obtained by the integral of the correlators and some exact sum rules results. The number with ${ }^{\dagger}$ refers to the amplitude in front of the logarithm.

Table 20 . Exact VEV of the four relevant operators in $\varphi_{4} \equiv t$ perturbation for $g_{4}<0$ (from [40]). The numbers with ${ }^{\dagger}$ refer to the amplitudes in front of the logarithm.

Table 21 . Estimated amplitudes in the vacancy density deformation for the three vacua. The number with ${ }^{\dagger}$ refers to the amplitude in front of the logarithm.

Table 22 . Amplitude ratios $R_{j k}^{2}=\frac{\Gamma_{j k}^{2+}}{\Gamma_{j k}^{2-}}$. The subscripts $2 \pm$ indicate the high or low temperature phases.

Table 23 . Universal ratios $\left(Q_{2}\right)_{j k}^{i}$ for $i, j, k=1,2^{+}, 2^{-}$.

Table 24 . Universal ratios $\left(R_{c}\right)_{j k}^{1},\left(R_{c}\right)_{j k}^{2-},\left(R_{c}\right)_{j k}^{3 a}$ and $\left(R_{c}\right)_{j k}^{3 b}$ where $2-$ indicates the low temperature phase and $3 a, 3 b$ are used to label the two different vacua $\left|0_{2}\right\rangle$ and $\left|0_{4}\right\rangle$ in the $g_{3} \varphi_{3}$ deformation. The other ratios are provided by the sum rule: $\left(R_{c}\right)_{j i}^{j}=\frac{\Delta_{j} \Delta_{i}}{\left(1-\Delta_{j}\right)^{2}}$.

Table 25 . Universal ratios $R_{\xi}^{i}$ and $\left(R_{A}\right)_{j}^{i}$ for $i, j=1,2^{-}, 2^{+}$.

Table 26 . Universal ratio $\left(R_{\chi}\right)_{j}^{i}$ for $i, j=1,2,3$. We also have $\left(R_{\chi}\right)_{j}^{j}=-\frac{\Delta_{j}}{\left(1-\Delta_{j}\right)}$ according to the sum rules and $\left(R_{\chi}\right)_{4}^{j}=0$ because $B_{44}=0$. 


\begin{tabular}{|ccc|}
\hline$\frac{3}{2}$ & $\frac{7}{16}$ & 0 \\
$\frac{6}{10}$ & $\frac{3}{80}$ & $\frac{1}{10}$ \\
$\frac{1}{10}$ & $\frac{3}{80}$ & $\frac{6}{10}$ \\
0 & $\frac{7}{16}$ & $\frac{3}{2}$ \\
\hline
\end{tabular}

Table 1:

\begin{tabular}{|l|l|}
\hline even $*$ even & \\
$\epsilon * \epsilon=[1]+c_{1}[t]$ & \\
$t * t=[1]+c_{2}[t]$ & \\
$\epsilon * t=c_{1}[\epsilon]+c_{3}\left[\varepsilon^{\prime \prime}\right]$ & $c_{1}=\frac{2}{3} \sqrt{\frac{\Gamma\left(\frac{4}{5}\right) \Gamma^{3}\left(\frac{2}{5}\right)}{\Gamma\left(\frac{1}{5}\right) \Gamma^{3}\left(\frac{3}{5}\right)}}$ \\
& $c_{2}=c_{1}$ \\
\hline even $*$ odd & $c_{3}=\frac{3}{7}$ \\
$\epsilon * \sigma^{\prime}=c_{4}[\sigma]$ & $c_{4}=\frac{1}{2}$ \\
$\epsilon * \sigma=c_{4}\left[\sigma^{\prime}\right]+c_{5}[\sigma]$ & $c_{5}=\frac{3}{2} c_{1}$ \\
$t * \sigma^{\prime}=c_{6}[\sigma]$ & $c_{6}=\frac{3}{4}$ \\
$t * \sigma=c_{6}\left[\sigma^{\prime}\right]+c_{7}[\sigma]$ & $c_{7}=\frac{1}{4} c_{1}$ \\
& $c_{8}=\frac{7}{8}$ \\
\hline odd $*$ odd & $c_{9}=\frac{1}{56}$ \\
$\sigma^{\prime} * \sigma^{\prime}=[1]+c_{8}\left[\varepsilon^{\prime \prime}\right]$ & \\
$\sigma^{\prime} * \sigma=c_{4}[\epsilon]+c_{6}[t]$ & \\
$\sigma * \sigma=[1]+c_{5}[\epsilon]+c_{7}[t]+c_{9}\left[\varepsilon^{\prime \prime}\right]$ & \\
\hline
\end{tabular}

Table 2: 


\begin{tabular}{rlll|}
$\sigma$ & $=\left[\frac{3}{80}, \frac{3}{80}\right]=\Phi$ & magnetization \\
$\epsilon$ & $=\left[\frac{1}{10}, \frac{1}{10}\right]=\Phi^{2}$ & energy \\
$\sigma^{\prime}$ & $=\left[\frac{7}{16}, \frac{7}{16}\right]=\Phi^{3}$ & sub-magnetization \\
$t$ & $=\left[\frac{6}{10}, \frac{6}{10}\right]=\Phi^{4}$ & vacancy density \\
$\varepsilon^{\prime \prime}$ & $=\left[\frac{3}{2}, \frac{3}{2}\right]=\Phi^{6}$ & irrelevant
\end{tabular}

Table 3:

\begin{tabular}{|c|c|c|c|c|c|c|}
\hline & $g_{1}$ & $g_{2}^{+}$ & $g_{2}^{-}$ & $g_{3}$ & $g_{4}^{+}$ & $g_{4}^{-}$ \\
\hline QFT & $\begin{array}{c}\text { Non- } \\
\text { Integrable }\end{array}$ & $\begin{array}{c}\text { integrable } \\
E_{7} \\
\text { (high-temp) }\end{array}$ & $\begin{array}{c}\text { integrable } \\
E_{7} \\
\text { (low-temp) }\end{array}$ & $\begin{array}{c}\text { integrable } \\
\text { (kinks) }\end{array}$ & $\begin{array}{c}\text { integrable } \\
\text { (massless } \\
\text { flow) }\end{array}$ & $\begin{array}{c}\text { integrable } \\
\text { (Susy } \\
\text { kinks) }\end{array}$ \\
\hline
\end{tabular}

Table 4:

\begin{tabular}{|c||c|c|c|c||}
\hline & $B_{11}$ & $B_{21}$ & $B_{31}$ & $B_{41}$ \\
\hline \hline$g_{1}>0($ num $)$ & $-1.539(6)$ & $1.35(6)$ & $-1.5(5)$ & $1.9(2)$ \\
\hline \hline
\end{tabular}

Table 5: 


\begin{tabular}{|c||c|c|c|c||}
\hline & $\varphi_{1}$ & $\varphi_{2}$ & $\varphi_{3}$ & $\varphi_{4}$ \\
\hline \hline$\langle 0|\varphi| 1\rangle_{1}$ & $-0.52(0)$ & $1.1(7)$ & $-4 .(9)$ & $7 .(4)$ \\
\hline$\langle 0|\varphi| 2\rangle_{1}$ & $-0.2(1)$ & $0.5(6)$ & $-3 .(3)$ & $5 .(8)$ \\
\hline
\end{tabular}

Table 6:

\begin{tabular}{|lcc||lcc|}
\hline$\left(C_{11}^{1}\right)_{1}^{(1)}$ & $=$ & 2 screening ops. & $\left(C_{11}^{3}\right)_{1}^{(1)}$ & $=$ & $-0.018583 \ldots$ \\
\hline$\left(C_{13}^{1}\right)_{1}^{(1)}$ & $\approx$ & $-0.482(1)$ & $\left(C_{13}^{3}\right)_{1}^{(1)}$ & $\approx$ & $0.395(0)$ \\
\hline$\left(C_{33}^{1}\right)_{1}^{(1)}$ & $=$ & $-0.214849 \ldots$ & $\left(C_{33}^{3}\right)_{1}^{(1)}$ & $=$ & 0 \\
\hline$\left(C_{12}^{0}\right)_{1}^{(1)}$ & $=$ & $-0.112093 \ldots$ & $\left(C_{12}^{2}\right)_{1}^{(1)}$ & $\approx$ & $0.517(2)$ \\
\hline$\left(C_{12}^{4}\right)_{1}^{(1)}$ & $\approx$ & $-0.015(0)$ & $\left(C_{14}^{0}\right)_{1}^{(1)}$ & $=$ & $-2.548155 \ldots$ \\
\hline$\left(C_{14}^{2}\right)_{1}^{(1)}$ & $\approx$ & $0.260(1)$ & $\left(C_{14}^{4}\right)_{1}^{(1)}$ & $=$ & 2 screening ops. \\
\hline$\left(C_{23}^{0}\right)_{1}^{(1)}$ & $=$ & $-2.816773 \ldots$ & $\left(C_{23}^{2}\right)_{1}^{(1)}$ & $=$ & $0.683830 \ldots$ \\
\hline$\left(C_{23}^{4}\right)_{1}^{(1)}$ & $=$ & $0.3787045 \ldots$ & $\left(C_{34}^{0}\right)_{1}^{(1)}$ & $=$ & $-0.922183 \ldots$ \\
\hline$\left(C_{34}^{2}\right)_{1}^{(1)}$ & $=$ & $0.259270 \ldots$ & $\left(C_{34}^{4}\right)_{1}^{(1)}$ & $=$ & $-0.665160 \ldots$ \\
\hline$\left(C_{22}^{1}\right)_{1}^{(1)}$ & $\approx$ & $0.266(0)$ & $\left(C_{22}^{3}\right)_{1}^{(1)}$ & $=$ & $-2.215418 \ldots$ \\
\hline$\left(C_{24}^{1}\right)_{1}^{(1)}$ & $\approx$ & $-1.54(9)$ & $\left(C_{24}^{3}\right)_{1}^{(1)}$ & $=$ & $0.504471 \ldots$ \\
\hline$\left(C_{44}^{1}\right)_{1}^{(1)}$ & $=$ & 2 screening ops. & $\left(C_{44}^{3}\right)_{1}^{(1)}$ & $=$ & 0 \\
\hline
\end{tabular}

Table 7: 


\begin{tabular}{|c|c|c|c|}
\hline Susceptibilities & Integration & TCSA & Sum Rule \\
\hline$\Gamma_{11}^{1}$ & $0.05(7)$ & $0.059(6)$ & 0.06 \\
\hline$\Gamma_{12}^{1}$ & $-0.13(6)$ & $-0.139(7)$ & -0.1396 \\
\hline$\Gamma_{13}^{1}$ & $0.69(6)$ & $0.68(7)$ & 0.70 \\
\hline$\Gamma_{14}^{1}$ & $-1.2(1)$ & $-1.1(4)$ & $-1.2(0)$ \\
\hline$\Gamma_{22}^{1}$ & $0.31(7)$ & $0.32(7)$ & \\
\hline$\Gamma_{23}^{1}$ & $-1.7(3)$ & $-1.6(7)$ & \\
\hline$\Gamma_{24}^{1}$ & $3 .(0)$ & $2 .(8)$ & \\
\hline$\Gamma_{33}^{1}$ & $15 .(3)$ & & \\
\hline$\Gamma_{34}^{1}$ & $3.76(9)^{\dagger}$ & & \\
\hline$\Gamma_{44}^{1}$ & $-15 .(5)^{*}$ & & \\
\hline
\end{tabular}

Table 8:

\begin{tabular}{|l|l|l|}
\hline$m_{1}=M$ & 1 & odd \\
$m_{2}=2 M \cos \left(\frac{5 \pi}{18}\right)$ & 1.28557 & even \\
$m_{3}=2 M \cos \left(\frac{\pi}{9}\right)$ & 1.87938 & odd \\
$m_{4}=2 M \cos \left(\frac{\pi}{18}\right)$ & 1.96961 & even \\
$m_{5}=4 M \cos \left(\frac{\pi}{18}\right) \cos \left(\frac{\pi}{9}\right)$ & 2.53208 & even \\
$m_{6}=4 M \cos \left(\frac{2 \pi}{9}\right) \cos \left(\frac{\pi}{9}\right)$ & 2.87938 & odd \\
$m_{7}=4 M \cos \left(\frac{\pi}{18}\right) \cos \left(\frac{\pi}{9}\right)$ & 3.70166 & even \\
\hline
\end{tabular}

Table 9: 


\begin{tabular}{|c||c|c|c|c||}
\hline & $B_{12}$ & $B_{22}$ & $B_{32}$ & $B_{42}$ \\
\hline \hline$g_{2}>0$ (num) & 0 & $-1.46(6)$ & 0 & $3 .(4)$ \\
\hline$g_{2}>0$ (exact) & 0 & $-1.46839 \ldots$ & 0 & $3.70708 \ldots$ \\
\hline$g_{2}<0$ (num) & $\pm 1.59(0)$ & $1.46(6)$ & $\pm 2.3(8)$ & $3 .(5)$ \\
\hline$g_{2}<0$ (exact) & $\pm 1.59427 \ldots$ & 1.46839 & $\pm 2.45205 \ldots$ & $3.70708 \ldots$ \\
\hline
\end{tabular}

Table 10:

\begin{tabular}{|c||c|c|c|c||}
\hline & $\varphi_{1}$ & $\varphi_{2}$ & $\varphi_{3}$ & $\varphi_{4}$ \\
\hline \hline$\left\langle 0\left|\varphi_{i}\right| 1\right\rangle_{2+}$ & $0.78(2)$ & 0 & $-6 .(4)$ & 0 \\
\hline$\left\langle 0\left|\varphi_{i}\right| 2\right\rangle_{2+}$ & 0 & $1.1(9)$ & 0 & $-11 .(1)$ \\
\hline$\left\langle 0\left|\varphi_{i}\right| 3\right\rangle_{2+}$ & $0.2(4)$ & 0 & $-4 .(3)$ & 0 \\
\hline$\left\langle 0\left|\varphi_{i}\right| 4\right\rangle_{2+}$ & 0 & $0.5(9)$ & 0 & $-8 .(7)$ \\
\hline
\end{tabular}

Table 11:

\begin{tabular}{|c||c|c|c|c||}
\hline & $\varphi_{1}$ & $\varphi_{2}$ & $\varphi_{3}$ & $\varphi_{4}$ \\
\hline \hline$\left\langle 0\left|\varphi_{i}\right| 1\right\rangle_{2-}$ & $0.50(5)$ & $1.1(9)$ & $6.3(5)$ & $11 .(1)$ \\
\hline$\left\langle 0\left|\varphi_{i}\right| 2\right\rangle_{2-}$ & 0 & $0.5(9)$ & 0 & $8 .(7)$ \\
\hline
\end{tabular}

Table 12: 


\begin{tabular}{|ccc||llc|}
\hline$\left(C_{11}^{0}\right)_{2}^{(1)}$ & $=$ & $0.223579 \ldots$ & $\left(C_{11}^{2}\right)_{2}^{(1)}$ & $=$ & $0.266530 \ldots$ \\
\hline$\left(C_{11}^{4}\right)_{2}^{(1)}(m r)$ & $\approx$ & $0.1510 . .\left(\ln (m r)+b_{1}(m)\right)$ & $\left(C_{13}^{0}\right)_{2}^{(1)}$ & $=$ & -2.007437 \\
\hline$\left(C_{13}^{2}\right)_{2}^{(1)}$ & $=$ & $0.677665 \ldots$ & $\left(C_{13}^{4}\right)_{2}^{(1)}$ & $=$ & $0.181313 \ldots$ \\
\hline$\left(C_{33}^{0}\right)_{2}^{(1)}$ & $=$ & 0 & $\left(C_{33}^{2}\right)_{2}^{(1)}$ & $=$ & 0 \\
\hline$\left(C_{33}^{4}\right)_{2}^{(1)}(m r)$ & $=$ & $2.3561 . .\left(\ln (m r)+b_{2}(m)\right)$ & $\left(C_{12}^{1}\right)_{2}^{(1)}$ & $\approx$ & $0.230(2)$ \\
\hline$\left(C_{12}^{3}\right)_{2}^{(1)}$ & $=$ & $0.109817 \ldots$ & $\left(C_{23}^{1}\right)_{2}^{(1)}$ & $=$ & $-3.766576 \ldots$ \\
\hline$\left(C_{23}^{3}\right)_{2}^{(1)}$ & $=$ & $1.840967 \ldots$ & $\left(C_{14}^{1}\right)_{2}^{(1)}$ & $\approx$ & $-3.79(9)$ \\
\hline$\left(C_{14}^{3}\right)_{2}^{(1)}$ & $=$ & $1.029533 \ldots$ & $\left(C_{34}^{1}\right)_{2}^{(1)}$ & $=$ & $-1.412466 \ldots$ \\
\hline$\left(C_{34}^{4}\right)_{2}^{(1)}$ & $=$ & $0.545471 \ldots$ & $\left(C_{22}^{2}\right)_{2}^{(1)}$ & $=$ & $1.008826 \ldots$ \\
\hline$\left(C_{22}^{4}\right)_{2}^{(1)}$ & $=$ & 0 & $\left(C_{24}^{0}\right)_{2}^{(1)}$ & $\approx$ & $-4.19(0)$ \\
\hline$\left(C_{24}^{2}\right)_{2}^{(1)}$ & $=$ & 0 & $\left(C_{24}^{4}\right)_{2}^{(1)}(m r)$ & $\approx$ & $2.84(0)\left(\ln (m r)+b_{3}(m)\right)$ \\
\hline$\left(C_{44}^{2}\right)_{2}^{(1)}$ & $\approx$ & $0.222(1)$ & $\left(C_{44}^{4}\right)_{2}^{(1)}$ & $=$ & \\
\hline
\end{tabular}

Table 13:

\begin{tabular}{|c|c|c|c|}
\hline Susceptibilities & Integration & TCSA & Sum Rule \\
\hline$\Gamma_{11}^{2+}$ & $0.093(9)$ & $0.093(7)$ & \\
\hline$\Gamma_{12}^{2+}$ & 0 & 0 & 0 \\
\hline$\Gamma_{13}^{2+}$ & $-0.8(9)$ & $-0.8(8)$ & \\
\hline$\Gamma_{14}^{2+}$ & 0 & 0 & 0 \\
\hline$\Gamma_{22}^{2+}$ & $0.15(8)$ & $0.16(0)$ & $0.16315 \ldots$ \\
\hline$\Gamma_{23}^{2+}$ & 0 & 0 & 0 \\
\hline$\Gamma_{24}^{2+}$ & $-2 .(2)$ & $-2 .(1)$ & $-2.466 \ldots$ \\
\hline$\Gamma_{33}^{2+}$ & $16 .(5)$ & & \\
\hline$\Gamma_{34}^{2+}$ & 0 & 0 & 0 \\
\hline$\Gamma_{44}^{2+}$ & $-17 .(5)^{*}$ & & \\
\hline
\end{tabular}

Table 14: 


\begin{tabular}{|c|c|c|c|}
\hline Susceptibilities & Integration & TCSA & Sum Rule \\
\hline$\Gamma_{11}^{2-}$ & $0.026(2)$ & $0.026(7)$ & \\
\hline$\Gamma_{12}^{2-}$ & $\pm 0.06(3)$ & $\pm 0.06(6)$ & $\pm 0.0662 \ldots$ \\
\hline$\Gamma_{13}^{2-}$ & $0.4(4)$ & $0.4(2)$ & \\
\hline$\Gamma_{14}^{2-}$ & $\pm 0.8(8)$ & $\pm 0.8(1)$ & \\
\hline$\Gamma_{22}^{2-}$ & $0.15(8)$ & $0.16(1)$ & $0.16315 \ldots$ \\
\hline$\Gamma_{23}^{2-}$ & $\pm 1.1(2)$ & $\pm 1.1(0)$ & $\pm 1.1145 \ldots$ \\
\hline$\Gamma_{24}^{2-}$ & $2 .(2)$ & $2 .(1)$ & $2.466 \ldots$ \\
\hline$\Gamma_{33}^{2-}$ & $12 .(6)$ & & \\
\hline$\Gamma_{34}^{2-}$ & $\pm 4.17378 \ldots{ }^{\dagger}$ & & \\
\hline$\Gamma_{44}^{2-}$ & $-17 .(5)^{*}$ & & \\
\hline \multicolumn{4}{|r}{} \\
\hline
\end{tabular}

Table 15:

\begin{tabular}{|c||c|c|}
\hline & $B_{i 3}$ for $|0\rangle=\left|0_{2}\right\rangle$ & $B_{i 3}$ for $|0\rangle=\left|0_{4}\right\rangle$ \\
\hline \hline$i=1$ & $0.68656 \ldots$ & $-1.79745 \ldots$ \\
\hline$i=2$ & $-0.78093 \ldots$ & $2.04451 \ldots$ \\
\hline$i=3$ & $-17.941605 \ldots$ & $-17.941605 \ldots$ \\
\hline$i=4$ & $2.69611 \ldots{ }^{\dagger}$ & $-7.05856 \ldots{ }^{\dagger}$ \\
\hline
\end{tabular}

Table 16:

\begin{tabular}{|c||c|c||}
\hline & $\varphi_{1}$ & $\varphi_{2}$ \\
\hline \hline$\left\langle 0_{2}\left|\varphi_{i}\right| 1\right\rangle_{3}$ & $-0.42(5)$ & $0.9(4)$ \\
\hline$\left\langle 0_{4}\left|\varphi_{i}\right| 2\right\rangle_{3}$ & $0.87(2)$ & $1.8(3)$ \\
\hline
\end{tabular}

Table 17: 


\begin{tabular}{|ccc||ccc|}
\hline$\left(C_{11}^{1}\right)_{3}^{(1)}(m r)$ & $=$ & $0.7189 . .\left(\ln (m r)+C_{1}(m)\right)$ & $\left(C_{11}^{3}\right)_{3}^{(1)}$ & $\approx$ & $-0.040(1)$ \\
\hline$\left(C_{12}^{0}\right)_{3}^{(1)}$ & $=$ & 8.79920 & $\left(C_{12}^{2}\right)_{3}^{(1)}$ & $=$ & -0.6571 \\
\hline$\left(C_{12}^{4}\right)_{3}^{(1)}$ & $=$ & 0.149171 & $\left(C_{22}^{1}\right)_{3}^{(1)}(m r)$ & $=$ & $2.8759 . . \ln \left(m r+C_{2}(m)\right)$ \\
\hline$\left(C_{22}^{3}\right)_{3}^{(1)}$ & $=$ & 0 & $\left(C_{14}^{0}\right)_{3}^{(1)}(m r)$ & $=$ & $-4.7123 . .\left(\ln (m r)+C_{3}(m)\right)$ \\
\hline
\end{tabular}

Table 18:

\begin{tabular}{|ccc||ccc|}
\hline \multicolumn{3}{|c||}{$\Gamma_{i j}^{3 a}$} & \multicolumn{3}{c|}{$\Gamma_{i j}^{3 b}$} \\
\hline$\Gamma_{11}^{3 a}=$ & $0.014(3)$ & $\Gamma_{11}^{3 b}$ & $=$ & $0.063(4)$ \\
\hline$\Gamma_{12}^{3 a}=$ & $-0.03(2)$ & $\Gamma_{11}^{3 b}$ & $=$ & $0.13(7)$ \\
\hline$\Gamma_{22}^{3 a}=$ & $0.076(5)$ & $\Gamma_{22}^{3 b}$ & $=$ & $0.29(0)$ \\
\hline$\Gamma_{13}^{3 a}=$ & $-0.045770 \ldots$ & $\Gamma_{13}^{3 b}$ & $=$ & $0.11983 \ldots$ \\
\hline$\Gamma_{23}^{3 a}=$ & $0.138832 \ldots$ & $\Gamma_{23}^{3 b}$ & $=$ & $-0.36346 \ldots$ \\
\hline$\Gamma_{33}^{3 a}$ & $=$ & $13.954582 \ldots$ & $\Gamma_{33}^{3 b}$ & $=$ & $13.954582 \ldots$ \\
\hline$\Gamma_{34}^{3 a}=$ & $-2.875855 \ldots{ }^{\dagger}$ & $\Gamma_{34}^{3 b}$ & $=$ & $7.52914 \ldots$ \\
\hline
\end{tabular}

Table 19:

\begin{tabular}{|c||c|c|c|}
\hline & $B_{i 4}$ for $|0\rangle=\left|0_{-1}\right\rangle$ & $B_{i 4}$ for $|0\rangle=\left|0_{0}\right\rangle$ & $B_{i 4}$ for $|0\rangle=\left|0_{+1}\right\rangle$ \\
\hline \hline$i=1$ & $-1.975669 \ldots$ & 0 & $1.975669 \ldots$ \\
\hline$i=2$ & $2.668319 \ldots$ & $-2.668319 \ldots$ & $2.668319 \ldots$ \\
\hline$i=3$ & $-10.640138 \ldots{ }^{\dagger}$ & 0 & $10.640138 \ldots{ }^{\dagger}$ \\
\hline$i=4$ & 0 & 0 & 0 \\
\hline
\end{tabular}

Table 20: 


\begin{tabular}{|lcc||lll|}
\hline \multicolumn{3}{|c||}{$\left(\Gamma_{i j}^{4-}\right)_{0}$} & \multicolumn{1}{c||}{$\left(\Gamma_{i j}^{4-}\right)_{ \pm 1}$} \\
\hline$\left(\Gamma_{11}^{4-}\right)_{0}$ & $=$ & $0.00(5)$ & $\left(\Gamma_{11}^{4-}\right)_{ \pm 1}$ & $=$ & $0.00(5)$ \\
\hline$\left(\Gamma_{12}^{4-}\right)_{0}$ & $=$ & 0 & $\left(\Gamma_{12}^{4-}\right)_{ \pm 1}$ & $=$ & $0.16(0)$ \\
\hline$\left(\Gamma_{22}^{4-}\right)_{0}$ & $=$ & $4.4(9) 10^{-3}$ & $\left(\Gamma_{22}^{4-}\right)_{ \pm 1}$ & $=$ & $4.4(9) 10^{-3}$ \\
\hline$\left(\Gamma_{14}^{4-}\right)_{0}$ & $=$ & 0 & $\left(\Gamma_{14}^{4-}\right)_{ \pm 1}$ & $=$ & $\mp 0.1852198 \ldots$ \\
\hline$\left(\Gamma_{24}^{4-}\right)_{0}$ & $=$ & $0.6670799 \ldots$ & $\left.\Gamma_{24}^{4-}\right)_{ \pm 1}$ & $=$ & $-0.6670799 \ldots$ \\
\hline$\left(\Gamma_{34}^{4-}\right)_{0}$ & $=$ & 0 & $\left(\Gamma_{34}^{4-}\right)_{ \pm 1}$ & $=$ & $\mp 11.637651 \ldots$ \\
\hline$\left(\Gamma_{44}^{4-}\right)_{0}$ & $=$ & 0 & $\left(\Gamma_{44}^{4-}\right)_{ \pm 1}$ & $=$ & 0 \\
\hline
\end{tabular}

Table 21:

\begin{tabular}{|ccc||llc|}
\hline$R_{11}^{2}=3.5(4)$ & $R_{13}^{2}$ & $=$ & $-2.0(6)$ \\
\hline$R_{22}^{2}=1$ & $R_{24}^{2}$ & $=$ & -1 \\
\hline$R_{33}^{2}$ & $=1.3(0)$ & $R_{44}^{2}$ & $=$ & 1 \\
\hline
\end{tabular}

Table 22:

\begin{tabular}{|cc||c|c|}
\hline$\left(Q_{2}\right)_{2^{+} 1}^{1}=1.26(0)$ & $\left(Q_{2}\right)_{2^{-}}^{1}=1.88(4)$ \\
\hline$\left(Q_{2}\right)_{2^{+} 2^{+}}^{1}=1.97(3)$ & $\left(Q_{2}\right)_{2^{+} 2^{-}}^{1}=1.32(0)$ \\
\hline$\left(Q_{2}\right)_{11}^{2+}=1.5(6)$ & $\left(Q_{2}\right)_{11}^{2-}=0.44(2)$ \\
\hline$\left(Q_{2}\right)_{12^{-}}^{2+}=1.7(0)$ & & \\
\hline
\end{tabular}

Table 23: 


\begin{tabular}{|l|||ll|}
\hline$\left(R_{c}\right)_{22}^{1}=1.0(5) 10^{-2}$ & $\left(R_{c}\right)_{23}^{1}=4.8(5) 10^{-2}$ \\
\hline$\left(R_{c}\right)_{24}^{1}=6 .(7) 10^{-2}$ & $\left(R_{c}\right)_{33}^{1}=3 .(8) 10^{-1}$ \\
\hline$\left(R_{c}\right)_{34}^{1}=-7 .(6) 10^{-2}$ & $\left(R_{c}\right)_{44}^{1}=-2 .(5) 10^{-1}$ \\
\hline \hline$\left(R_{c}\right)_{11}^{2-}=1.7(0) 10^{-3}$ & $\left(R_{c}\right)_{14}^{2-}=2.3(3) 10^{-2}$ \\
\hline$\left(R_{c}\right)_{13}^{2-}=1.7(9) 10^{-2}$ & $\left(R_{c}\right)_{33}^{2-}=3 .(4) 10^{-1}$ \\
\hline$\left(R_{c}\right)_{34}^{2-}=7.4912 \ldots 10^{-2}$ & $\left(R_{c}\right)_{44}^{2-}=-2 .(0) 10^{-1}$ \\
\hline \hline$\left(R_{c}\right)_{11}^{3 a}=4.2(3) 10^{-1}$ & $\left(R_{c}\right)_{11}^{3 b}=2.7(3) 10^{-1}$ \\
\hline$\left(R_{c}\right)_{12}^{3 a}=8.3(2) 10^{-1}$ & $\left(R_{c}\right)_{12}^{3 b}=5.2(0) 10^{-1}$ \\
\hline$\left(R_{c}\right)_{22}^{3 a}=1.7(5)$ & $\left(R_{c}\right)_{22}^{3 b}=9.6(8) 10^{-1}$ \\
\hline
\end{tabular}

Table 24:

\begin{tabular}{|cc||cc|}
\hline$R_{\xi}^{1}=7.55(7) 10^{-2}$ & & \\
\hline$R_{\xi}^{2+}$ & $=1.07(8) 10^{-1}$ & $R_{\xi}^{2-}$ & $=8.38(9) 10^{-2}$ \\
\hline \hline$\left(R_{A}\right)_{2+}^{1}=0$ & $\left(R_{A}\right)_{2-}^{1}=3.91(8) 10^{-2}$ \\
\hline$\left(R_{A}\right)_{1}^{2+}=2.95(8) 10^{-1}$ & $\left(R_{A}\right)_{1}^{2-}=8.26(0) 10^{-1}$ \\
\hline
\end{tabular}

Table 25:

\begin{tabular}{|cc||cc|}
\hline$\left(R_{\chi}\right)_{2}^{1}=1.1(9) 10^{-1}$ & $\left(R_{\chi}\right)_{3}^{1}=4.2(5) 10^{-1}$ \\
\hline$\left(R_{\chi}\right)_{1}^{2-}=4 .(0) 10^{-2}$ & $\left(R_{\chi}\right)_{3}^{2-}=4 .(0) 10^{-1}$ \\
\hline$\left(R_{\chi}\right)_{1}^{3 a}=2 .(0) 10^{-11}$ & $\left(R_{\chi}\right)_{1}^{3 b}=1.8(7)$ \\
\hline$\left(R_{\chi}\right)_{2}^{3 a}=3 .(3) 10^{-4}$ & $\left(R_{\chi}\right)_{2}^{3 b}=2.7(8)$ \\
\hline
\end{tabular}

Table 26: 


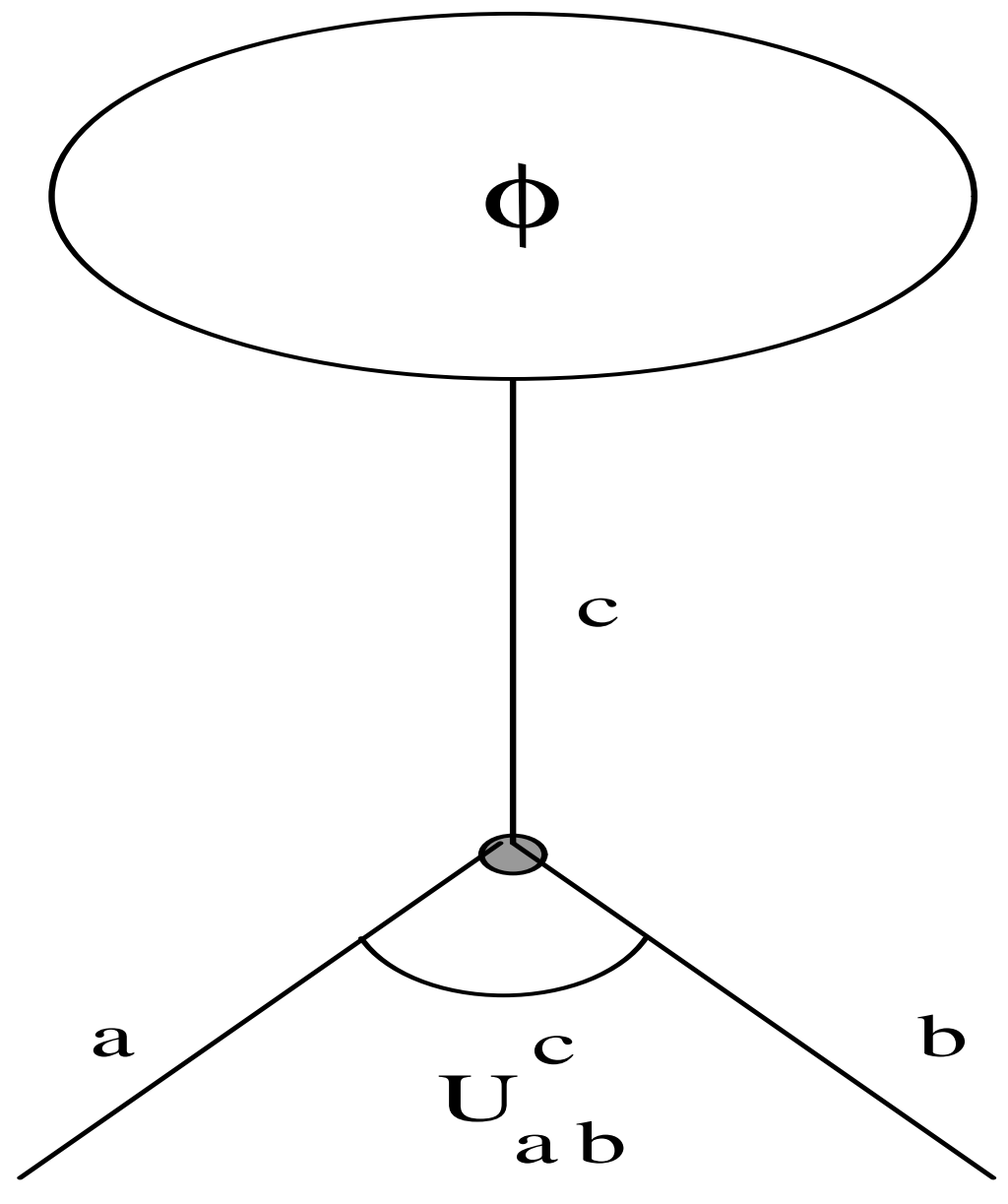

Figure 1: Diagram responsible for a single-pole in the two-particle Form Factors 


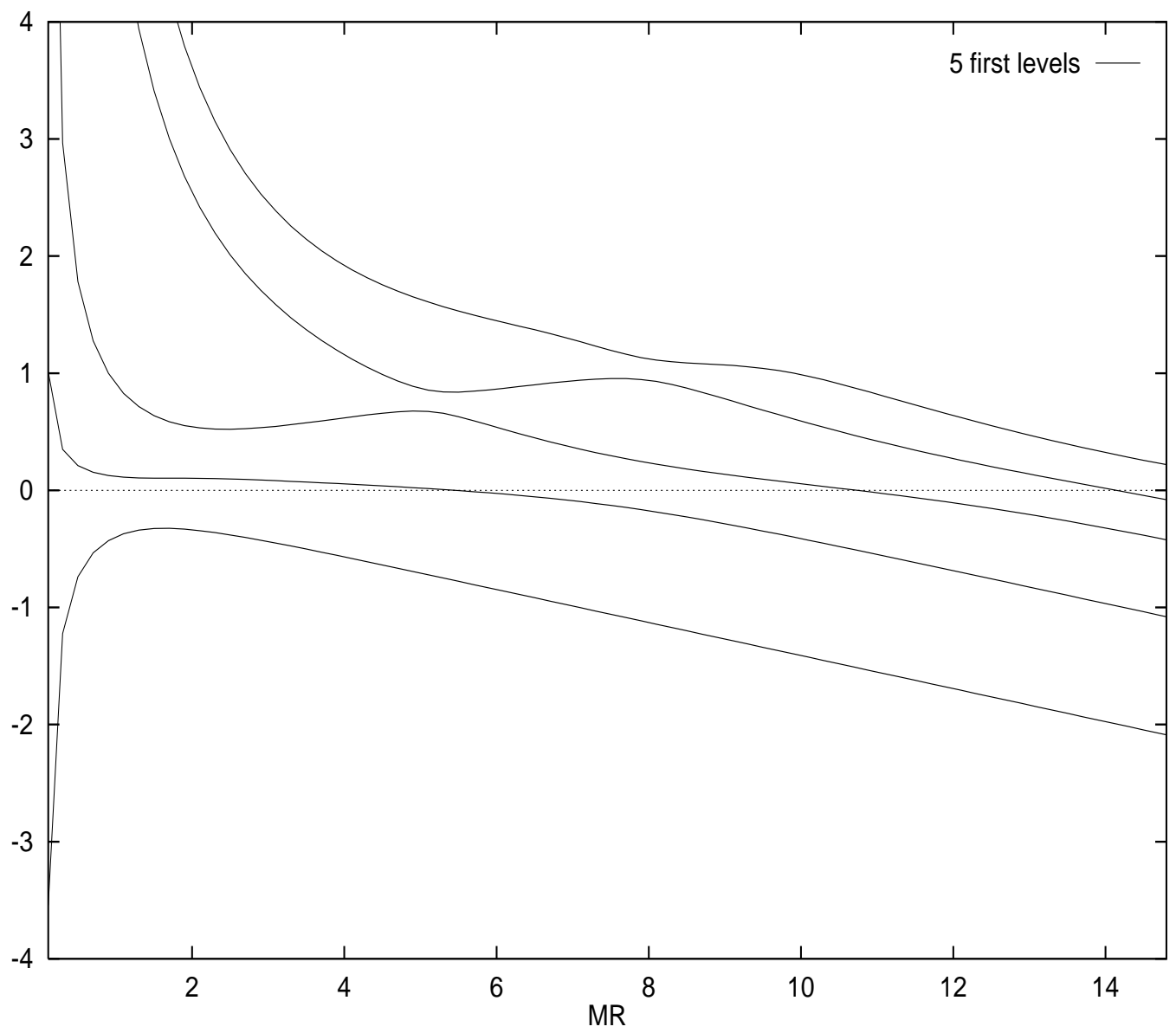

Figure 2: The first 5 energy levels of the TIM perturbed by the magnetic field as a function of $M R$. 


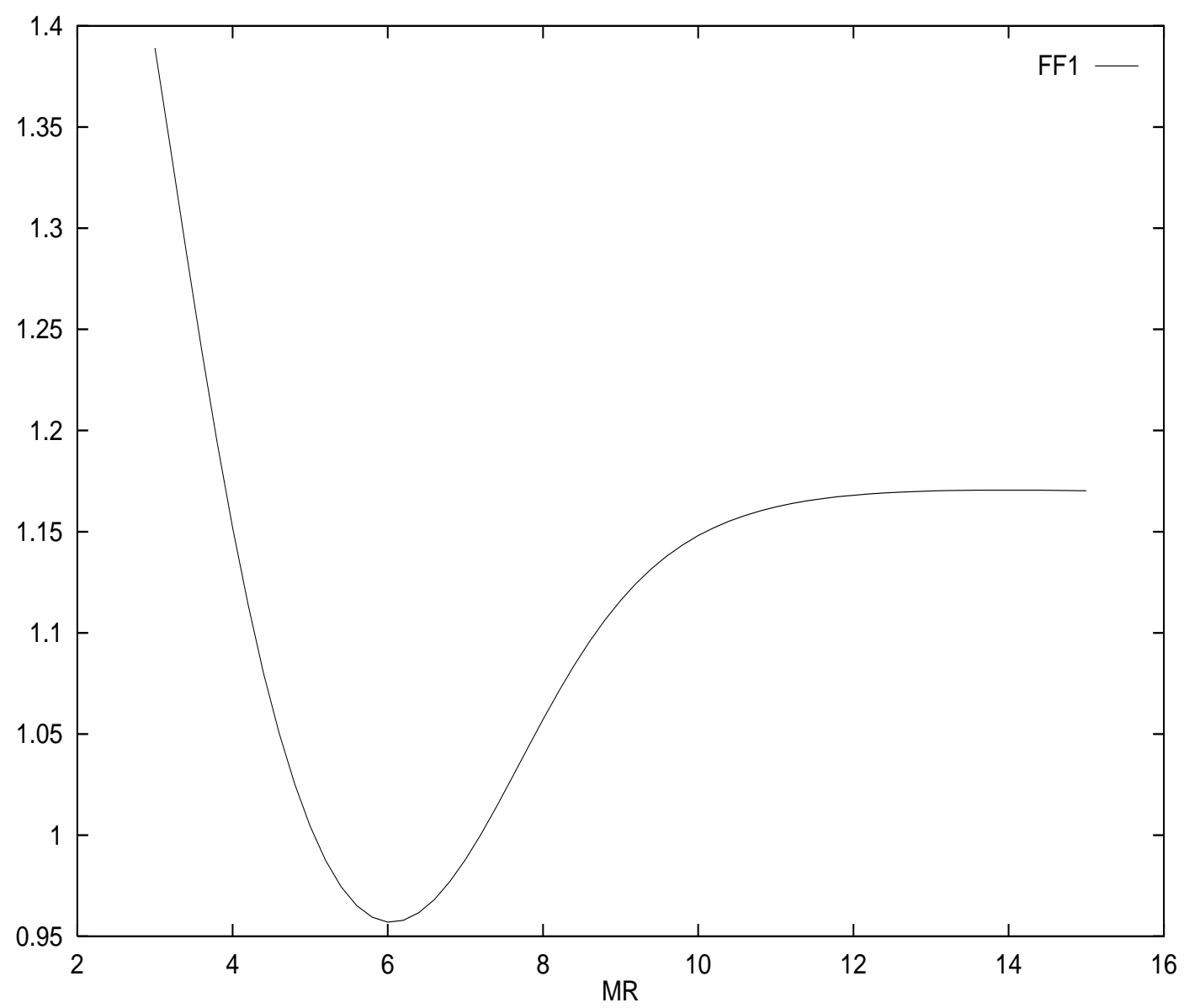

Figure 3: One-particle FF $\left\langle 0\left|\varphi_{2}(0)\right| A_{1}\right\rangle_{1}$ as a function of $M R$ for the magnetic perturbation of the TIM. 


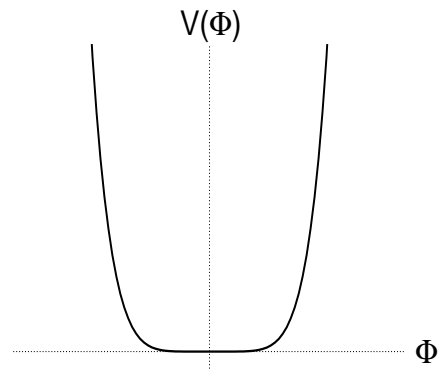

(a)

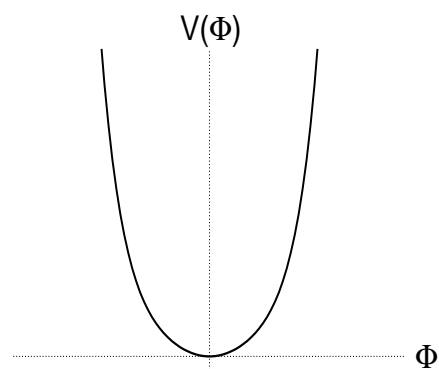

(d)

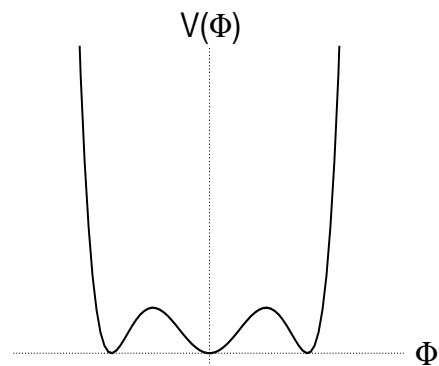

(g)

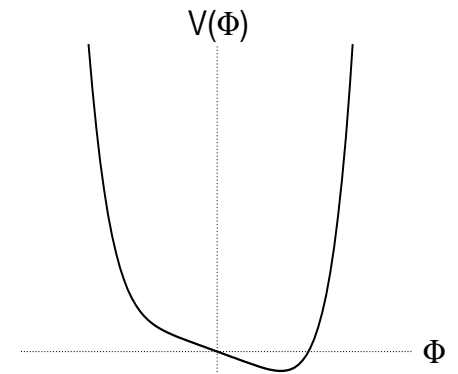

(b)

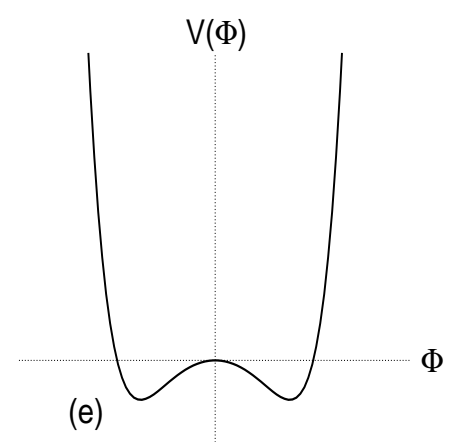

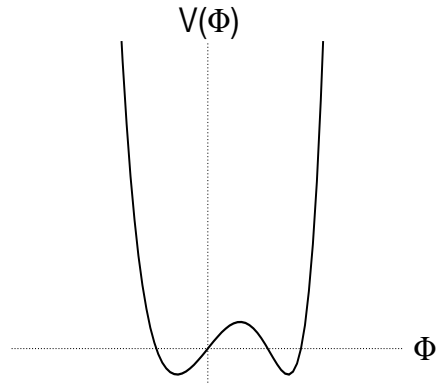

(c)

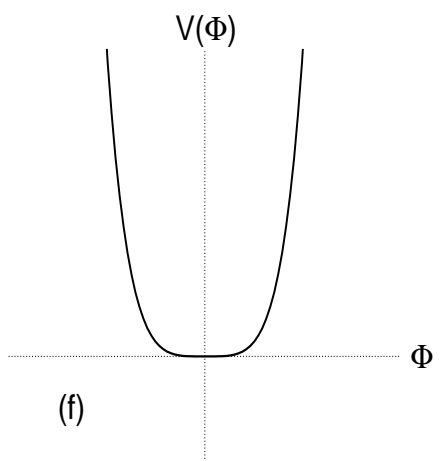

Figure 4: Effective LG potentials associated to the $\Phi^{6}$ theory: (a) at the tricritical point and perturbed by $(\mathrm{b})$ the leading magnetic field $\left(g_{1} \neq 0\right)$, (c) the sub-leading magnetic field $g_{3}>0$, the leading energy density with (d) $g_{2}>0$ or (e) $g_{2}<0$, the vacancy density with (f) $g_{4}>0$ or (g) $g_{4}<0$. 


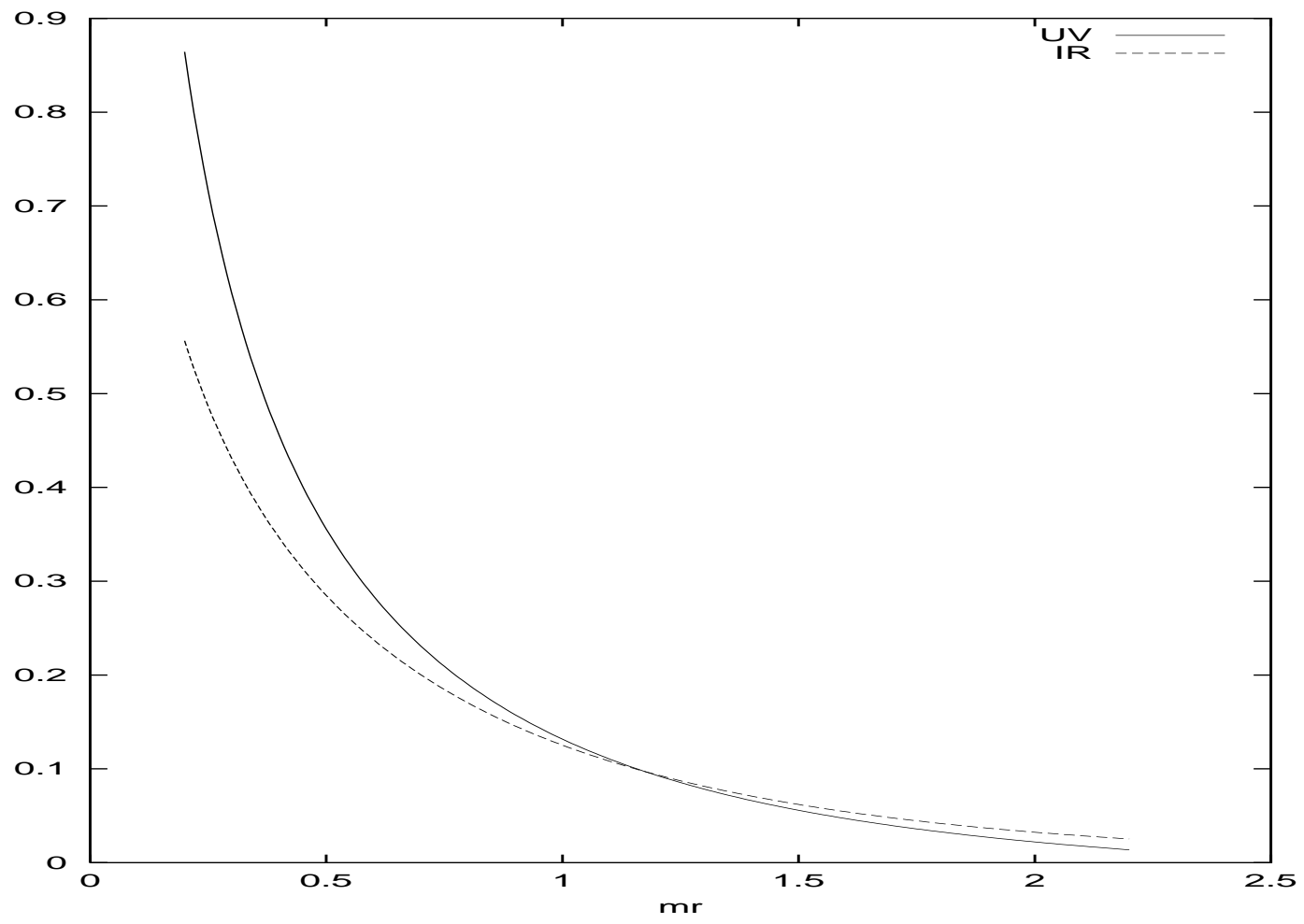

Figure 5: The continuous line gives the UV approximation of the correlator $\left\langle\varphi_{2}(x) \varphi_{2}(0)\right\rangle_{1}$ whereas the dashed line depicts the IR approximation. An overlap of the two curves is observed around $m r \sim 1$. 


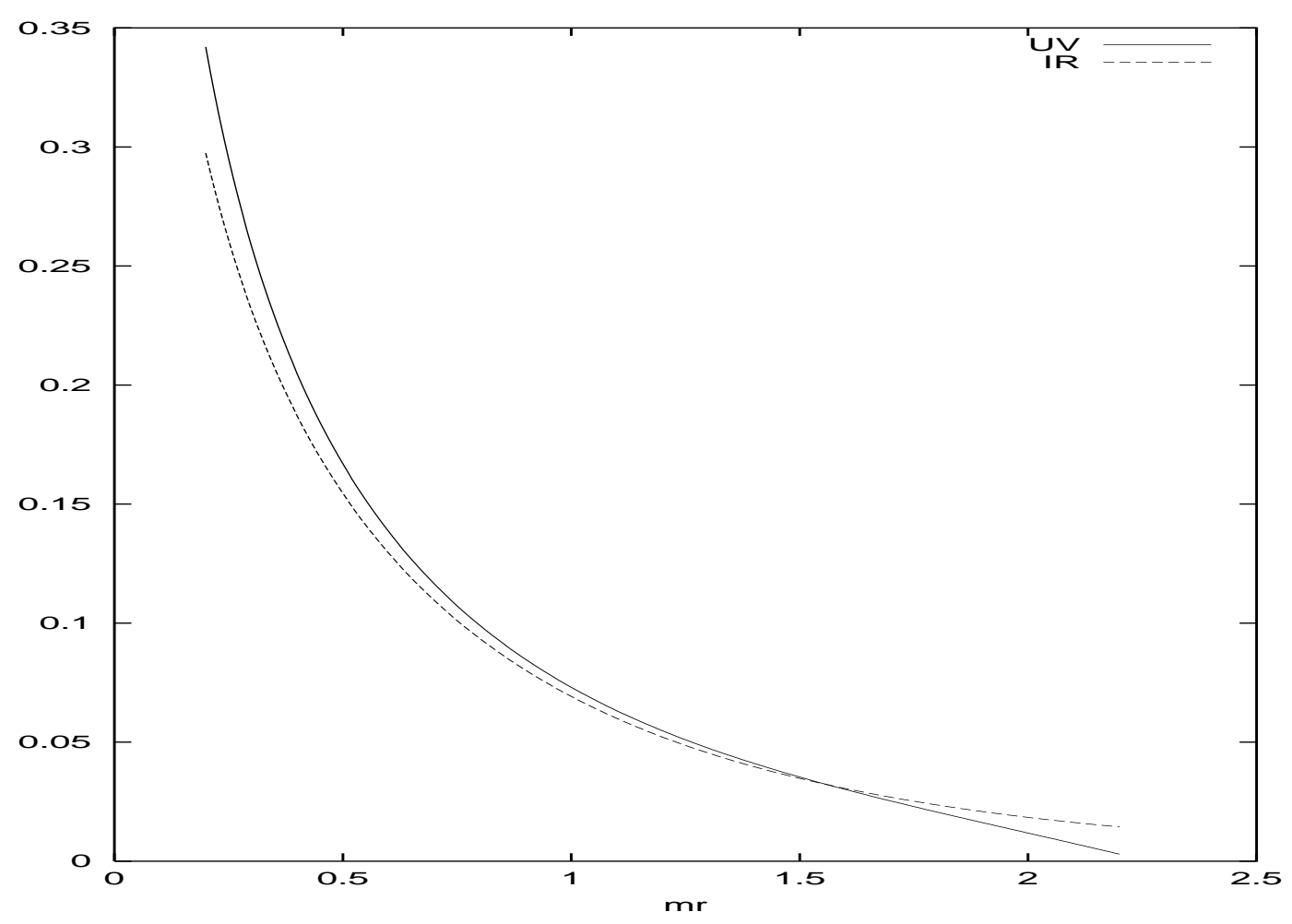

Figure 6: The UV approximation of the correlator $\left\langle\varphi_{1}(x) \varphi_{1}(0)\right\rangle_{2}$ is given by the continuous line whereas its IR approximation is given by the dashed line. An overlap of the curves is observed around $m r \sim 1$. 